\title{
Rapid ecological and evolutionary divergence during a poleward range expansion
}

Monica M. Sheffer ${ }^{1 *}$, Linda Zander ${ }^{1}$, Brian Schulze ${ }^{1 \#}$, Pierick Mouginot ${ }^{2}$, Thomas Naef ${ }^{3}$, Jürgen Kreyling ${ }^{4}$, Rosemary G. Gillespie ${ }^{5}$, Katharina J. Hoff ${ }^{6,7}$, Stefan Prost ${ }^{8,9,10,11}$, Henrik Krehenwinkel ${ }^{12}$ \& Gabriele Uhl ${ }^{1}$

${ }^{1}$ General and Systematic Zoology, Zoological Institute and Museum, University of Greifswald, 17489 Greifswald, Germany

${ }^{2}$ PSL Research University, CNRS-EPHE-UPVD, USR 3278 CRIOBE, 66360, Perpignan, France

${ }^{3}$ Applied Zoology and Nature Conservation, Zoological Institute and Museum, University of Greifswald, 17489 Greifswald, Germany

${ }^{4}$ Experimental Plant Ecology, Institute for Botany and Landscape Ecology, University of Greifswald, 17489 Greifswald, Germany

${ }^{5}$ Department of Environmental Science Policy and Management, University of California Berkeley, 94720 Berkeley, CA, USA

${ }^{6}$ Institute of Mathematics and Computer Science, University of Greifswald, 17489 Greifswald, Germany

${ }^{7}$ Center for Functional Genomics of Microbes, University of Greifswald, 17489 Greifswald, Germany

${ }^{8}$ Department of Behavioural and Cognitive Biology, University of Vienna, 1090 Vienna, Austria

${ }^{9}$ Konrad Lorenz Institute of Ethology, University of Veterinary Medicine, A-1160 Vienna, Austria

${ }^{10}$ Natural History Museum Vienna, Central Research Laboratories, 1010 Vienna, Austria

${ }^{11}$ South African National Biodiversity Institute, National Zoological Gardens of South Africa, Pretoria 0001, South Africa

${ }^{12}$ Department of Biogeography, University of Trier, 54296 Trier, Germany

*Indicates corresponding author

\#Current affiliation: Animal Ecology, Zoological Institute and Museum, University of Greifswald, 17489 Greifswald, Germany 


\begin{abstract}
Anthropogenic climate change is rapidly altering ecosystems, driving range shifts, range contractions, dwindling population sizes and local extinctions in many species. Some species, however, are expanding their ranges and seem to benefit from warming temperatures. This is the case for the wasp spider, Argiope bruennichi, which has undergone a range expansion from its historic range in the Mediterranean ("core"), now reaching as far as the Baltic states and Scandinavia ("edge"). The rate of this range expansion cannot be attributed to climate change alone, and it has been hypothesized that adaptive introgression lent the genetic variation upon which selection could act, enabling the rapid range expansion. In the present study, we first quantify the degree of local adaptation and phenotypic plasticity in cold tolerance in edge relative to core populations, and secondly investigate genomic and phenotypic turnover across the proposed introgression zone. With a reciprocal transplant common garden experiment, we provide strong support for the hypothesis that edge populations are locally adapted to colder winter conditions. We also find evidence of seasonal plasticity in the core populations, while edge populations have lost this plasticity. Our genome-wide analysis, using a combination of $\mathrm{F}_{\text {ST }}$ outlier and genetic-environment association tests, supports the hypothesis that adaptive introgression played a role in environmental adaptation.
\end{abstract}

\title{
Keywords
}

Range expansion, local adaptation, phenotypic plasticity, cold tolerance, spider, adaptive introgression 


\section{Introduction}

Organisms experience changing environments from season to season, but also in the long term, and less predictably, facing global climate change. Global change is massively altering ecosystems, leading to extinction events, dwindling population sizes, and range contractions in many species worldwide. However, some species seem to profit from these changes, displaying dramatic poleward range expansions (reviewed in i.e. Parmesan et al., 1999; Hickling et al., 2006; Chen et al., 2011). Such range expansions have long been explained by environmental change alone. Recent work shows that rangeexpanding populations differentiate from core populations during the process of expansion. Environmental change may make a new habitat suitable in one dimension, such as sufficiently warm summer temperatures for development, while other dimensions, such as colder winter temperatures at the expanding front, may require genetic adaptation or adaptive phenotypic plasticity (Sexton et al., 2009; Colautti \& Barrett, 2013). This is the case, for example, in the range-expanding damselfly, Ischnura elegans, which has maintained its heat tolerance but altered its lower thermal tolerances, likely through evolutionary adaptation, during a poleward range expansion (Lancaster et al., 2015, 2016). The rapid establishment and phenotypic differentiation of range-expanding species offers the opportunity to investigate the roles of plasticity and evolutionary adaptation in responses to novel environments, and to obtain information on the underlying genomic basis of adaptation. This is especially true for species with passive dispersal, such as seeds or spiders dispersing in the wind, with little to no control over where they land.

Phenotypic plasticity, the potential for a single genotype to produce differing phenotypes in response to differing environmental conditions (see Fordyce, 2006; Whitman \& Agrawal, 2009), can facilitate range expansion, enabling dispersing organisms to persist wherever they arrive. However, even if plasticity is sufficient for initial survival and establishment, local adaptation may subsequently arise, i.e. if there is a cost to plasticity (Ghalambor et al., 2007; Auld, Agrawal \& Relyea, 2010; Hoffmann \& Bridle, 2021). Common garden and reciprocal transplant experiments allow for disentangling plastic and adaptive responses, by manipulating the environment while controlling for genetic background. Such experiments have demonstrated that local genetic adaptation is widespread in nature (i.e. Clausen, Keck \& Hiesey, 1941; Leimu \& Fischer, 2008; Hereford, 2009; Fraser et al., 2011). However, the relative importance of novel mutations or standing genetic variation providing the substrate for selection, enabling genetic adaptation, cannot be derived from those experiments (Barrett \& Schluter, 2008; Hedrick, 2013; Hoban et al., 2016), and is rarely studied in conjunction with the relative importance of adaptation and phenotypic plasticity in organismal responses to novel environments.

In theory, adaptation from standing genetic variation should take place more quickly than adaptation from novel mutations. This has a number of reasons; for instance, variants which may be adaptive under novel environments are already present in the gene pool and occur at reasonably high frequencies, while novel mutations seldom arise and start at low frequencies, which are more susceptible to genetic drift (reviewed in Barrett \& Schluter, 2008). A third avenue through which adaptive variants can arise in a genepool is via introgression, admixture or intraspecific hybridization of previously isolated populations. Traditionally, hybridization has often been associated with deleterious effects, such as outbreeding depression (Muhlfeld et al., 2009). More recently, however, studies have increasingly begun to identify cases of adaptive introgression. For example, introgression plays a strong role in wing coloration in the Heliconius butterfly mimicry complex (Zhang et al., 2016; Moest et al., 2020). Adaptive introgression can be especially rapid, when it involves the sharing of alleles that are under very strong selection in a novel 
environment, as has been demonstrated in the case of rapid pesticide resistance in an African mosquito species (Norris et al., 2015). Another interesting example for adaptive introgression is the increased fitness at higher altitudes found in Tibetan humans, which has been shown to stem from introgression of Denisovan-like DNA into humans (Huerta-Sánchez et al., 2014; Racimo et al., 2015).

Argiope bruennichi is an orb-weaving spider species with a Palearctic distribution, which has undergone a rapid latitudinal range expansion within Europe in the last century (Follner \& Klarenberg, 1995; Algo, 2010; Krehenwinkel \& Tautz, 2013; Krehenwinkel, Rödder \& Tautz, 2015; Wawer et al., 2017). Krehenwinkel \& Tautz (2013) attributed the range expansion to climate-mediated adaptive introgression, with individuals from eastern Asia mixing with the European gene pool via Russia, providing genetic variation that enabled rapid adaptation to the colder, previously unsuitable conditions in northern Europe. Indeed, as a consequence of their latitudinal range expansion, individuals in the expanding populations prefer colder temperatures (Krehenwinkel \& Tautz, 2013) and have a colder climatic niche, as they must endure much more extreme winter temperatures and higher seasonality than their counterparts in the core of the range (Krehenwinkel et al., 2015). What remains unclear is whether the observed differences are due to phenotypic plasticity or genetic adaptation, and where, precisely, adaptive introgression occurred geographically and within the genome.

To address the first hypothesis, we set out to quantify the level of genetic adaptation and phenotypic plasticity in edge relative to core populations in a suite of life history and physiological traits which may enhance their ability to endure the colder winter conditions at the expanding edge. We did this using a reciprocal transplant common garden experiment, overwintering juvenile spiders ("spiderlings") from replicated core or edge populations in either native or foreign conditions. At two time points, mid-winter and post-winter, we measured their survival, mass, body size, and several cold tolerance metrics: namely supercooling point, lower lethal temperature, and chill coma recovery time.

To address the second hypothesis, we investigated phenotypic traits and genomic patterns across latitude. Our sampling scheme focused on core and edge populations and the area of suspected introgression. We quantified how adult phenotypes (body size, clutch size, hatching success, and pigmentation) vary with latitude. We assessed population genomic patterns across latitude, and scanned for signatures of adaptive introgression in the genome, to test if introgression truly provided the substrate for selection enabling range expansion in this species.

\section{Material and Methods}

\section{Population samples}

We collected adult $A$. bruennichi females in a transect from southern France to Estonia, with dense sampling in Germany cutting across the admixture zone proposed by Krehenwinkel \& Tautz (2013) from July $29^{\text {th }}$-August $10^{\text {th }}, 2018$. We timed the collecting trip according to the mating season, when the females had mated (indicated by the lack of males, which had been cannibalized) but not yet laid egg sacs (indicated by the lack of egg sacs in the field). We filled in the gaps between southern France, Germany, and Estonia using DNA extracts from samples previously used in Krehenwinkel \& Tautz (2013). Similarly, we used DNA extracts from that study for outgroup populations from Russia and Japan (Figure 1). We aimed to collect 10 individuals per site, although we included populations with as few as three individuals when population densities were low. Our transect within Europe spans 15 degrees of latitude. Exact collecting locations with GPS coordinates and sample sizes can be found in Table 1. 
In addition to the $\sim 10$ animals per site collected from the NE-SW transect, we collected 94 more adult $A$. bruennichi females from the three sites in Estonia and 80 more from the four sites in southern France to increase the sample size for our reciprocal transplant common garden experiment (henceforth "common garden experiment").

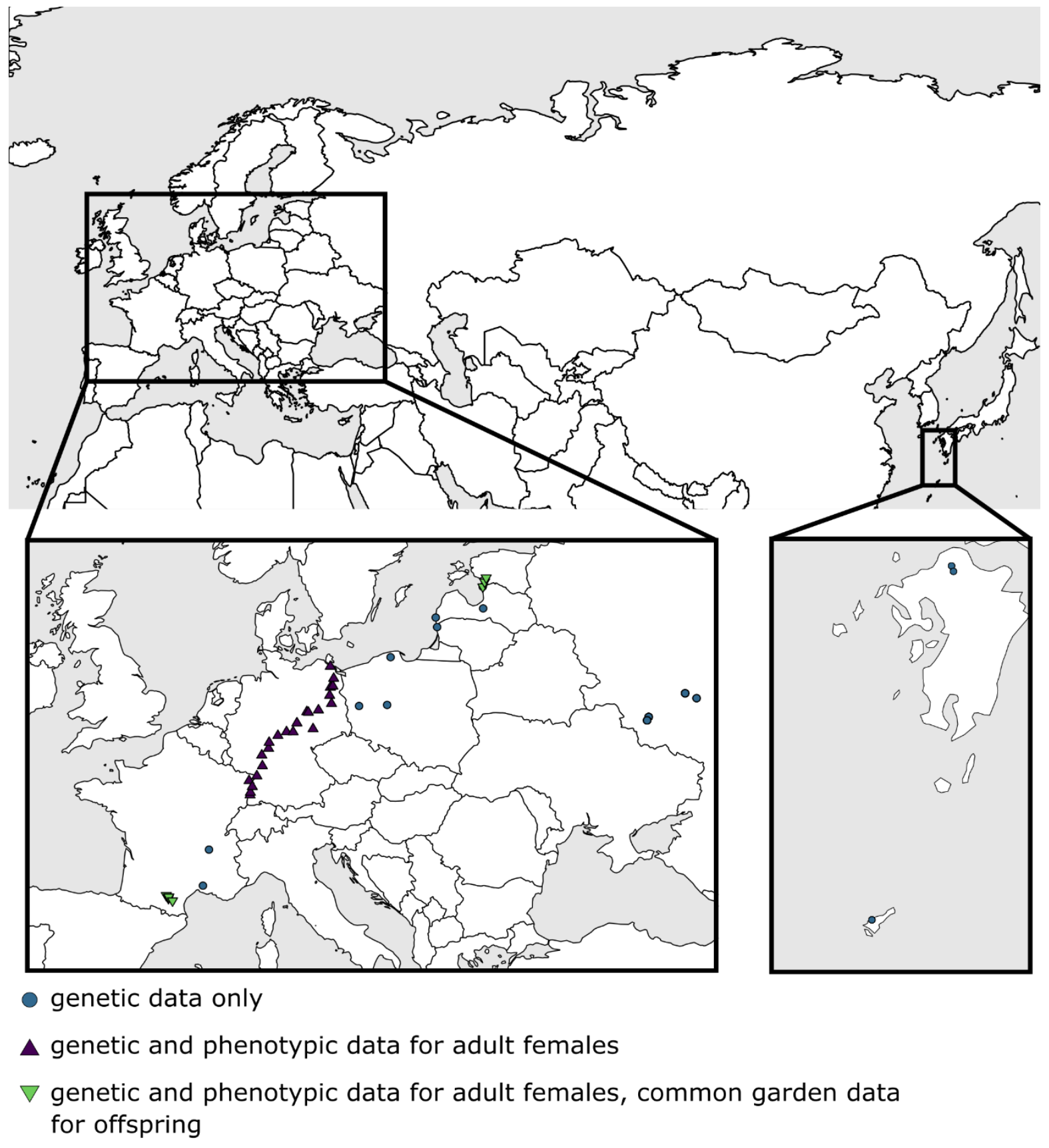

Figure 1. Collecting sites included in this study. Each point represents a collecting locality; point shape and color corresponds to the data collected at each site. 
bioRxiv preprint doi: https://doi.org/10.1101/2021.11.04.467261; this version posted November 4,2021 . The copyright holder for this preprint (which was not certified by peer review) is the author/funder, who has granted bioRxiv a license to display the preprint in perpetuity. It is made available under aCC-BY-ND 4.0 International license.

Table 1. Collecting sites included in this study. The "Use" column contains a sub-column for the two aspects of our study: the common garden experiment (CG) and the transect sampling. The transect sub-column contains either "P" (data for adult phenotypes) and/or " $G$ " (genetic data), or " $O$ " (outgroup genetic data). In the sample size column $(" N$ "), the number in [square brackets] indicates additional females collected to achieve reasonable sample sizes for the common garden experiment. Populations in bold text have data for both aspects (transect and common garden).

\begin{tabular}{|c|c|c|c|c|c|c|c|}
\hline \multirow{2}{*}{$\begin{array}{l}\text { Population } \\
\text { Number }\end{array}$} & \multirow{2}{*}{ Population Name } & \multicolumn{2}{|r|}{ Use } & \multirow{2}{*}{ Country } & \multirow{2}{*}{$\underline{\mathbf{N}}$} & \multirow{2}{*}{$\underline{\text { Latitude }\left({ }^{\circ} \mathrm{N}\right)}$} & \multirow{2}{*}{ Longitude $\left({ }^{\circ} \mathrm{E}\right)$} \\
\hline & & $\underline{\mathrm{CG}}$ & Transect & & & & \\
\hline 1 & Belflou & $\checkmark$ & $P \& G$ & France & $10[7]$ & 43.32287 & 1.786019 \\
\hline 2 & Casties & $\checkmark$ & $P \& G$ & France & $10[7]$ & 43.18677 & 1.939997 \\
\hline 3 & Mireval & $\checkmark$ & $P \& G$ & France & $10[51]$ & 43.25358 & 1.961534 \\
\hline 4 & Perry & $\checkmark$ & $P \& G$ & France & 10 [15] & 43.07072 & 2.231319 \\
\hline 5 & Nimes & - & $\mathrm{G}$ & France & 10 & 43.78 & 4.4 \\
\hline 6 & Lyon & - & $G$ & France & 10 & 45.48 & 4.83 \\
\hline 8 & Umkirch & - & $P \& G$ & Germany & 10 & 48.05299 & 7.758187 \\
\hline 9 & Kenzingen & - & $P \& G$ & Germany & 10 & 48.17536 & 7.783603 \\
\hline 10 & Offenburg & - & $P \& G$ & Germany & 10 & 48.44384 & 7.919677 \\
\hline 11 & Brumath & - & $P \& G$ & France & 11 & 48.72702 & 7.689943 \\
\hline 12 & Au Am Rhein & - & $P \& G$ & Germany & 10 & 48.95041 & 8.243427 \\
\hline 13 & Heidelberg & - & $P \& G$ & Germany & 10 & 49.42313 & 8.638357 \\
\hline 14 & Graefenhause & - & $P \& G$ & Germany & 10 & 49.93076 & 8.594512 \\
\hline 15 & Buedingen & - & $P \& G$ & Germany & 10 & 50.24868 & 9.078187 \\
\hline 16 & Schotten & - & $P \& G$ & Germany & 10 & 50.5284 & 9.117785 \\
\hline 17 & Bad Hersfeld & - & $P \& G$ & Germany & 10 & 50.85265 & 9.743685 \\
\hline 18 & Eisenach & - & $P \& G$ & Germany & 8 & 51.02786 & 10.35974 \\
\hline 19 & Gierstadt & - & $P \& G$ & Germany & 10 & 51.02483 & 10.82997 \\
\hline 20 & Kelbra & - & $P \& G$ & Germany & 10 & 51.44596 & 11.10118 \\
\hline 21 & Pegau & - & $P \& G$ & Germany & 10 & 51.16905 & 12.25069 \\
\hline 22 & Zeits & - & $P \& G$ & Germany & 4 & 51.97261 & 11.8048 \\
\hline 23 & Barby & - & P\&G & Germany & 3 & 51.94881 & 11.9164 \\
\hline 24 & Rabenstein & - & P\&G & Germany & 10 & 52.056 & 12.636 \\
\hline 25 & Schulzendorf & - & $P \& G$ & Germany & 10 & 52.36082 & 13.56633 \\
\hline 26 & Wandlitz & - & $P \& G$ & Germany & 10 & 52.74649 & 13.4346 \\
\hline 27 & Templin & - & $P \& G$ & Germany & 4 & 53.12951 & 13.48775 \\
\hline 28 & Mittenwald & - & $P \& G$ & Germany & 7 & 53.1869 & 13.66299 \\
\hline 29 & Strasburg & - & $P \& G$ & Germany & 10 & 53.54765 & 13.72238 \\
\hline 30 & Ludwigsburg & - & $P \& G$ & Germany & 10 & 54.11404 & 13.48225 \\
\hline 32 & Grodziszcze & - & G & Poland & 7 & 52.25 & 15.533 \\
\hline 33 & Poznan & - & $\mathrm{G}$ & Poland & 10 & 52.3 & 17.5333 \\
\hline 34 & Lebork & - & $\mathrm{G}$ & Poland & 10 & 54.55 & 17.78 \\
\hline 35 & Palanga & - & $\mathrm{G}$ & Lithuania & 10 & 55.97 & 21.1 \\
\hline 36 & Liepaja & - & $\mathrm{G}$ & Latvia & 10 & 56.42 & 21 \\
\hline 37 & Salaspils & - & $\mathrm{G}$ & Latvia & 10 & 56.85 & 24.38 \\
\hline 38 & Ainazi & - & $\mathrm{G}$ & Latvia & 10 & 57.87 & 24.35 \\
\hline 39 & Ikla & $\checkmark$ & $P \& G$ & Estonia & $10[6]$ & 57.87248 & 24.35843 \\
\hline 40 & Pulgoja & $\checkmark$ & P\&G & Estonia & $10[9]$ & 58.10143 & 24.48833 \\
\hline 41 & Paernu & $\checkmark$ & P\&G & Estonia & 10 [79] & 58.29702 & 24.59738 \\
\hline 42 & Selikhovy Dvory Village & - & 0 & Russia & 10 & 51.57 & 36.07 \\
\hline 43 & Khytor Stepnoy Village & - & 0 & Russia & 10 & 51.73333 & 36.18333 \\
\hline 44 & Donskoye Village & - & 0 & Russia & 10 & 52.85 & 38.78333 \\
\hline 45 & Yablonevoye Village & - & 0 & Russia & 3 & 52.61667 & 39.6 \\
\hline 46 & Amami oshima Island & - & $\mathrm{O}$ & Japan & 7 & 28.33 & 129.32 \\
\hline 47 & Amami oshima Island & - & $\mathrm{O}$ & Japan & 9 & 28.33 & 129.32 \\
\hline 48 & Fukuoka & - & 0 & Japan & 10 & 33.65 & 130.6833 \\
\hline
\end{tabular}




\section{Animal husbandry}

After collection, we brought the spiders into a climate controlled chamber at the University of Greifswald, Germany, where they produced one to two egg sacs under shared conditions ( $80 \%$ relative humidity, $26^{\circ} \mathrm{C}$ day temperature, $20^{\circ} \mathrm{C}$ night temperature, with 12 hours of full day, 10 hours of full night, and one hour to transition between the day and night temperatures). The spiders were stored in plastic containers (height: $13 \mathrm{~cm}$, diameter: $12 \mathrm{~cm}$ ) with a hole filled with cotton on the top for ventilation; additional humidity was provided by spraying the cotton with water every evening. We fed the spiders with adults or larvae of Lucilia caesar and Calliphora vomitoria flies twice a week.

After the period for oviposition was over, the adult females were anesthetized using $\mathrm{CO}_{2}$, then preserved in pure ethanol and stored at $-80^{\circ} \mathrm{C}$. Females that died of natural causes in the lab were not used for sequencing, as they were not immediately preserved, and thus the DNA could have degraded over night until they were checked the next day.

\section{Common garden experiment}

\section{Timing of experiments}

Egg sacs were laid over a period of one month and 15 days, between August 4, 2018 and September 19, 2018. We will henceforth refer to the number of days between collection and oviposition as "oviposition latency." In the field, A. bruennichi females lay their eggs into round flask-shaped egg sacs, placed $\sim 20 \mathrm{~cm}$ above the ground, in the grass (Wawer et al., 2017; MM Sheffer, personal observation). Females do not guard or tend their egg sacs for extended periods of time, and a single female can produce multiple egg sacs; we refer to the eggs in each egg sac as a "clutch." Females in our study laid up to two egg sacs. The day following oviposition, we placed the egg sacs into $5 \times 5 \times 3.5 \mathrm{~cm}$ plastic boxes with mesh on two sides, and moved them into climate cabinets programmed with the same day/night conditions under which they were laid, until the onset of winter treatments. Eggs develop, hatch and molt within two to four weeks following oviposition (Leborgne \& Pasquet, 2005; Schneider, Fromhage \& Uhl, 2005; Welke \& Schneider, 2010). These molted spiderlings overwinter within the egg sac. Here, it is important to note that because the egg sacs were laid before we began the winter treatments, and hatching occurs shortly after oviposition, clutch size and hatching success will not be affected by the winter treatments.

On October $1^{\text {st }}, 2018$, we changed the climate conditions in the climate cabinets, one with a "warm" winter treatment, corresponding to the natural conditions in southern France (climate cabinet: Panasonic Pflanzenzucht- und Klimatestkammer MLR-352H, Ewald Innovationstechnik GmbH, Bad Nenndorf, Germany), and the other a "cold" winter treatment, corresponding to the natural conditions in Estonia (climate cabinet: Percival Tieftemperaturkammer LT-36VL mit Fernkondensator, CLF PlantClimatics $\mathrm{GmbH}$, Wertingen, Germany). The egg sacs from each origin (France "core", or Estonia "edge") were randomly assigned to warm or cold winter treatments, while ensuring approximately equal sample sizes per origin and treatment (Table 2). For details on the winter regimens, see Supplemental File 1: Figure S1. All egg sacs from a single mother (up to two) were placed into the same winter treatment.

At mid-winter (February 4-March 19, 2019) and post-winter (April 4-May 19, 2019) intervals, we opened a subset of the egg sacs and counted the number of eggs, dead spiderlings, and living spiderlings, and performed cold tolerance experiments, described below. Egg sacs opened at mid-winter were opened six 
months ( $183+/-2$ days) after the oviposition date, while post-winter egg sacs were opened eight months (243+/- 1 days) after oviposition.

Table 2. Sample sizes for the common garden experiment. Egg sacs laid by adult female spiders from French (core) and Estonian (edge) populations were placed into either warm or cold winter treatments. The egg sacs were opened at mid- (February-March) or post-winter (April-May). Some, but not all, mothers laid two egg sacs.

\begin{tabular}{|c|c|c|c|c|}
\hline \multicolumn{3}{|c|}{ Experimental group } & \multirow[b]{2}{*}{$\# 1^{\text {st }}$ Egg sacs } & \multirow[b]{2}{*}{$\# 2^{\text {nd }}$ Egg sacs } \\
\hline Origin & $\underline{\text { Winter treatment }}$ & Season & & \\
\hline Core & Warm & Mid-Winter & 31 & 16 \\
\hline Core & Warm & Post-Winter & 31 & 7 \\
\hline Core & Cold & Mid-Winter & 30 & 8 \\
\hline Core & Cold & Post-Winter & 28 & 9 \\
\hline Edge & Warm & Mid-Winter & 33 & 26 \\
\hline Edge & Warm & Post-Winter & 30 & 20 \\
\hline Edge & Cold & Mid-Winter & 31 & 19 \\
\hline Edge & Cold & Post-Winter & 30 & 10 \\
\hline
\end{tabular}

For egg sacs with sufficient numbers of living spiderlings, we allotted 15 spiderlings for body size measurements, 20 spiderlings for lower lethal temperature measurements, 10 spiderlings for chill coma recovery time measurements, and 8 spiderlings for supercooling point measurements. In some families with low survival, small clutch size, or low hatching success, slightly fewer spiderlings were used in some measurements. Precise descriptions of the measurements taken follow below.

\section{Response variables for common garden experiment}

\section{Life history traits and survival}

We calculated the clutch size of egg sac as the sum of eggs, dead spiderlings, and living spiderlings. Hatching success was calculated as the sum of dead and living spiderlings ("hatched" spiderlings) divided by the total clutch size. Survival was calculated as the number of living spiderlings divided by the total number of hatched spiderlings.

\section{Mass and body size (spiderlings)}

After counting the spiderlings, we immediately took the mass of 15 individual spiderlings per clutch using a Sartorius ME5 scale (Sartorius AG, Goettingen, Germany). After weighing, the spiderlings were stored individually in $80 \%$ ethanol at room temperature. Later, the preserved spiderlings were removed from ethanol, and the first leg on the right side was removed and photographed using a Zeiss Discovery V20 Microscope in combination with a Zeiss AxioCam MRc camera with a $0.63 X$ objective (Zeiss AG, Oberkochen, Germany). From the photos, we measured the length from the proximal end of the patella to the distal end of the tibia ("Tibia-Patella length"), using the Carl Zeiss AxioVision SE64 Software (v.4.9.1.2, Zeiss AG, Oberkochen, Germany). Tibia-patella length is a commonly-used proxy for body size in spiders (Uhl \& Vollrath, 2000).

\section{Chill coma recovery time}

10 spiderlings per clutch were placed individually into a 3D-printed microwell plate. The wells were painted bright white with non-toxic water-based paint to increase the contrast between the well and the 
spiderlings. The wells were covered with a glass slide to prevent the spiderlings from escaping, and then the plate was placed into a $-9^{\circ} \mathrm{C}$ freezer for one hour, to induce chill coma. After one hour, the plate was carefully removed from the freezer, and placed into a climate chamber $\left(15^{\circ} \mathrm{C}, 80 \%\right.$ humidity $)$. The glass slide was changed to prevent fogging, and then the spiderlings were filmed in the plate for one hour using an iPhone 6 camera. Analysis of the videos was done manually, with the IDs obscured, by scanning through the video while watching a single spider, until the first movement. Then the video was re-watched at normal speed from a few seconds before the first movement, and the time of the very first movement (i.e. a leg twitch, shift in body position) was marked down as the chill coma recovery time, in seconds. All videos from the mid-winter experiments were re-watched to determine the repeatability of the measurements; the CCR time did not differ significantly between the first and second watching, therefore we did not re-watch the post-winter videos.

\section{Supercooling point}

An organism's supercooling point is the temperature at which the body water freezes. This point can be detected by the heat of crystallization released upon ice formation (Sinclair, Coello Alvarado \& Ferguson, 2015). In order to determine A. bruennichi supercooling points, eight spiderlings per clutch were placed individually into a pipette tip, which had been sealed at the tip. A thermocouple (Type K) was inserted into the pipette tip until it came into contact with the spiderling, and was then held in place with a piece of cotton, so that neither the spiderling nor the thermocouple could move. The pipette tips were placed into well-fitting holes drilled into a custom-made aluminum block, and the block was then placed into a dynamic climate chamber (Modell MKT 115, Binder GmbH, Tuttlingen, Germany). Temperature data from the thermocouples was gathered by Pico USB TC-08 thermocouple data loggers (Pico Technology, Cambridgeshire, United Kingdom) once every second for the duration of the trial. Trials started at $5^{\circ} \mathrm{C}$ and ramped down at a rate of approximately $-0.4^{\circ} \mathrm{C} /$ minute for a total of 150 minutes. Data was copied from the data logger output program into Excel, and then analyzed with a custom R script (Supplemental File 2). The supercooling point was calculated as the temperature immediately before a temperature increase above the threshold of $0.3^{\circ} \mathrm{C} /$ second. The threshold was determined by running the script on several sets of data with different thresholds; $0.3^{\circ} \mathrm{C} /$ second was sensitive enough to detect the more gradual peaks, but not so sensitive that background changes in temperature were recorded as supercooling points. Each batch of peaks with supercooling points marked was visually inspected before being entered into the final datasheet.

\section{Lower lethal temperature}

Of the 20 spiderlings taken from each clutch, 10 were used as test subjects, and 10 were used as a control group, to account for mortality unrelated to the test. The tube containing test spiderlings was placed into a freezer at either $-10^{\circ} \mathrm{C},-18^{\circ} \mathrm{C},-26^{\circ} \mathrm{C}$, or $-32^{\circ} \mathrm{C}$, for one hour, then placed into a climate chamber held at $15^{\circ} \mathrm{C}, 80 \%$ humidity, to recover. The control spiderlings were handled in the same way (carried to the freezer, but not placed inside, and then carried to the climate chamber), but remained at the recovery temperature for the duration of the test ( 2 hours). After the recovery period, the test and control tubes were opened (blind to treatment, ID, and test/control status) and the number of live and dead spiderlings was recorded. A spiderling was considered dead only if it did not respond to any stimulus (touch with a paintbrush or submersion in ethanol). Of the 1,738 spiderlings in control groups, only 15 died. 


\section{Statistical analysis of common garden data}

\section{Explanatory variables}

In order to assess phenotypic plasticity and genetic adaptation in the common garden experiment, our explanatory variables of central interest were "origin" (southern France or Estonia) and "winter treatment" (warm or cold). We also investigated seasonal differences with the explanatory variable "season" (mid-winter or post-winter). All starting models included the interaction of these three variables, except for clutch size and hatching success, as these could not be affected by the winter treatments (see "Timing of experiments," above). For the lower lethal temperature experiments, exposure temperature $\left(-10^{\circ} \mathrm{C},-18^{\circ} \mathrm{C},-26^{\circ} \mathrm{C}\right.$, or $\left.-32^{\circ} \mathrm{C}\right)$ was also included as an explanatory variable. Additional, potentially biologically relevant, variables were oviposition latency (time from collection to oviposition), mother's body size, clutch size, and whether the individual came from a first or second clutch. Continuous explanatory variables were centered and scaled in order to allow for interpretation of effects on the same scale (Schielzeth, 2010).

\section{Random effects}

The population from which a spider was collected (three populations in Estonia, four populations in southern France) was included as a random effect. For response variables measured at the individual spiderling level, the clutch (first or second) and mother's ID were included as nested random effects within population - specified in the model as (1 | Population / Mother ID / Clutch). If measurements were done at the clutch level, only population was included as a random effect (1 | Population).

\section{Modeling approach}

We analyzed all common garden data using (generalized) linear mixed models in $\mathrm{R}$, using the package 'glmmTMB' (Brooks et al., 2017) for non-Gaussian distributions and the package 'Ime4' for Gaussian distributions (Bates et al., 2015). Details of the models for each response variable follow below.

For the data on clutch size, we used a negative binomial distribution with default link function and linear parameterization ("nbinom1" in R). Hatching success was modeled using a betabinomial distribution with default link. The hatching success data showed zero inflation due to oviposition latency, so we included a zero inflation formula to account for the excess of zero hatching success in late-laid egg sacs. We used a betabinomial distribution with default link for the survival model. As for hatching success, we also found zero inflation due to oviposition latency (an excess of zero survival in late-laid egg sacs). Therefore, we included oviposition latency as a zero inflation formula. Mass and body size were modeled using a Gaussian distribution.

The distribution of chill coma recovery times was highly skewed, and attempts to find an appropriate distribution family and model to handle the skewness proved unsuccessful. Therefore, we logtransformed the raw data, allowing us to model with a Gaussian distribution. Due to the characteristic multimodal distribution of supercooling points (i.e. Sinclair et al., 2003), we followed the common practice in the field, and split the data into "low group" (LG) and "high group" (HG) supercooling points at $-25.5^{\circ} \mathrm{C}$, chosen according to visual inspection of a break in the distribution of the raw data (Supplemental File 1: Figure S2). The LG SCPs had a normal distribution, and were modeled as a single response variable with a Gaussian distribution. The HG SCPs were much rarer, and did not allow for modeling directly. Thus, we calculated the per-clutch ratio of HG:LG SCPs, to test whether any of our treatment groups had a tendency toward more HG SCPs. This HG:LG comparison was modeled with a binomial distribution and logit link. 
Finally, the lower lethal temperature data were modeled with a betabinomial distribution and default link. These data were recorded as the number surviving or dying within a subset of one clutch. The exposure temperature was added as an explanatory variable, along with all other aforementioned explanatory variables.

For each full starting model, we performed a backward stepwise model selection using AIC, with random factors always included in the compared models. We did not remove any fixed effects, only the interactions, according to AIC. The model with the lowest AIC was then assessed further. For models with a $\triangle \mathrm{AIC}$ of less than four, we always proceeded with the most parsimonious (i.e. least complex) model within those criteria. Model assumptions were checked using the R package 'DHARMa', which uses simulated residuals to provide easily interpretable checks of assumptions for many model and distribution types (Hartig, 2021).

Where possible, we used the Nakagawa \& Schielzeth method (Nakagawa \& Schielzeth, 2013) to calculate a pseudo- $R^{2}$, implemented in the 'performance' R package (Lüdecke et al., 2021), to assess how much of the variation in the data was explained by the full model (conditional pseudo- $R^{2}$ ), and by the model without random effects (marginal pseudo- $\mathrm{R}^{2}$ ). The Nakagawa \& Schielzeth method is not reliable for betabinomial models, thus we used a crude calculation suggested in the 'glmmTMB' FAQs (https://bbolker.github.io/mixedmodels-misc/glmmFAQ.html) to understand the goodness of fit for our betabinomial models (hatching success, overwintering survival, and lower lethal temperature); precisely, we calculated the squared correlation of the predicted values with the observed values of the response variable (calculated in $\mathrm{R}$ : cor(model.response(model.frame(model)), predict(model, type = "response") $)^{\wedge} 2$ ). This does not provide a comparison of conditional and marginal $R^{2}$, thus for betabinomial models we only report the conditional $\mathrm{R}^{2}$. We refer to "significant" effects as those where the $95 \%$ confidence interval $(\mathrm{Cl})$ does not cross zero.

To visualize the results, we used the 'ggpredict' function from the 'ggeffects' R package (Lüdecke, 2018) to compute estimated marginal means and $95 \% \mathrm{Cl}$ of the response variable for specific model terms. This allows us to visualize results on the observed scale, making biological interpretation more straightforward. Likewise, although within the model continuous fixed effects were centered and scaled, we plotted the model estimates with the unscaled values.

\section{Historical climate data across transect}

We extracted historical climate data for all collecting sites along the transect, excluding the outgroups in Russia and Japan, using an R script and data for the 19 standard bioclimatic variables downloaded from WorldClim at 30 second $\left(\sim 1 \mathrm{~km}^{2}\right)$ resolution (Fick \& Hijmans, 2017). To summarize and reduce the number of environmental variables in downstream analyses, we summarized the 19 variables into fewer dimensions using a principal components analysis (PCA) using the R package 'FactoExtra' (Kassambara \& Mundt, 2020) (Supplemental File 3), following the recommendation of Hoban et al. (2016). The first five principle components (PCs) accounted for $97.6 \%$ of the variation in the climate data, and were used as explanatory variables for phenotype-environment and genotype-environment associations.

\section{Transect data - adult female phenotypes}

For the females from the study by Krehenwinkel \& Tautz (2013), we have genetic data but do not have phenotype data, as we directly used their previously extracted DNA and the physical specimens were not 
available. Thus, we collected phenotype data for the females collected in 2018 from southern France, Germany and Estonia, described in detail below.

\section{Adult female body size}

We removed and photographed the first leg on the right side from each female using a Zeiss Discovery V20 Microscope in combination with a Zeiss AxioCam MRc camera with a 0.63X objective (Zeiss AG, Oberkochen, Germany). From the photos, we measured tibia-patella length as a proxy for body size, as described above for spiderlings.

\section{Opisthosoma pigmentation}

To characterize the pigmentation of the dorsal opisthosoma, we photographed each female in the field, using an Olympus Tough F2.0 camera with a ring light flash attachment mounted on a wooden box lined with aluminum foil and blue paper bottom. The spiders were anesthetized using $\mathrm{CO}_{2}$ so that we could position them for the photograph. When the camera was mounted, no light could enter the box other than the diffused light from the ring light, thus standardizing the lighting conditions in the field. The images were processed in Gimp (v.2.10): we cut out the opisthosoma, added a blue background, and reduced the number of colors in the image to six, using the "posterize" function. The six colors were then characterized as dark (black, red, green, or violet) or light (white or yellow), and we calculated the number of light and dark pixels per spider in $\mathrm{R}$.

\section{Clutch size and hatching success}

Egg sacs laid by German females were opened approximately two months after oviposition (64 +/- 13 days). As above, we calculated the clutch size as the sum of eggs, dead spiderlings, and living spiderlings. Hatching success was calculated as the sum of dead and living spiderlings ("hatched" spiderlings) relative to the total clutch size. For the transect, we only include data from the first clutch.

\section{Statistical analysis of transect phenotype data}

To test if the female traits (body size, pigmentation, clutch size and hatching success) varied with the environment, we used generalized linear mixed models in $\mathrm{R}$, using the package 'glmmTMB' (Brooks et al., 2017). The female traits were used as response variables, and the five environmental principle components were used as explanatory variables. Body size was modeled with a Gaussian distribution, clutch size with a negative binomial distribution with quadratic parameterization ("nbinom2" in R), pigmentation with a binomial (light vs dark pixels) distribution, and hatching success with a betabinomial distribution. Sampling site was included as a random effect. All models fit linear assumptions.

\section{Transect data - population genomics and signatures of adaptation}

\section{Library preparation and sequencing}

We extracted genomic DNA from the four left legs of the adult female spiders using a plate extraction protocol, as follows: the legs were placed into single well of a 96 -well plate containing $3 \mathrm{~mm}$ steel beads, cell lysis buffer (made of $10 \mathrm{mM}$ Tris $\mathrm{pH} 8,100 \mathrm{mM} \mathrm{NaCl}, 10 \mathrm{mM}$ EDTA pH 8, 0.5\% SDS, and double distilled water) and Proteinase K. The plate was then shaken in a GenoGrinder 2010 (OPS Diagnostics) at 1,200 hz for 2 minutes for physical tissue lysis, followed by overnight incubation at $55^{\circ} \mathrm{C}$. The next day, RNAse $\mathrm{A}$ was added to digest RNA. We then added $\mathrm{NaCl}$ to precipitate the proteins, and spun the plate down. We retained the supernatant, containing DNA, and discarded the insoluble protein phase. We used isopropanol to precipitate the DNA from the supernatant, then washed the DNA pellet twice with $70 \%$ 
ethanol. The ethanol was allowed to evaporate, leaving only DNA. The extracted DNA was rehydrated in $50 \mu \mathrm{l}$ of TE Buffer. DNA quantification was carried out using a Qubit fluorimeter (Thermo Scientific, Waltham, USA) according to the manufacturer's protocol.

We prepared dual-indexed libraries using a double digest restriction-enzyme-associated DNA sequencing (ddRADseq) library preparation protocol, based on the Peterson method (Peterson et al., 2012), as adapted in Maas et al. (2018), with 500ng of DNA as starting material for most samples. Some samples started with lower input, but library preparation succeeded nonetheless. We digested the DNA using the restriction enzymes NlallI (frequent cutter) and SbfI-HF (rare cutter). These enzymes were chosen based on their GC content, to avoid over-digestion of the AT-rich genome. We tested the digestion with in silico digestions of the A. bruennichi genome assembly (Sheffer et al., 2021b) using SimRAD (Lepais \& Weir, 2014), as well as testing digestions in the lab according to the Peterson protocol. We indexed each individual with unique barcodes, then pooled the individuals into 19 libraries of 24 individuals each. Another round of indexing was performed, to give each library of individuals a unique identifier. We quantified the indexed libraries using RT-qPCR, then pooled all of the libraries together. We used a Sage Science Pippen Prep to size select 475-696 bp fragments (including internal adapters), with a peak at 570 bp. The fragment size range was confirmed with a Bioanalyzer High Sensitivity chip (Agilent). We then sequenced $150 \mathrm{bp}$ single-end reads on three lanes of Illumina HiSeq 4000 at UC Berkeley's Vincent J. Coates Genomics Sequencing Lab.

\section{Sequence processing and SNP filtering}

We demultiplexed the raw reads based on the individual barcode sequences, then used Trimmomatic (v.0.39) (Bolger, Lohse \& Usadel, 2014) to remove adapter sequences. We mapped the reads of each sample onto the genome assembly using BWA-MEM (v.0.7.12-r1039) with default settings (Li, 2013), then sorted and indexed the mapped reads using SAMtools sort and SAMtools index (SAMtools v.1.3.1) (Li et al., 2009).

We generated a VCF file for all samples using SAMtools mpileup. We then filtered the SNP data using SNPcleaner (v.2.24) (Fumagalli et al., 2014). SNPs were retained for downstream analyses if they had a minimum coverage of $3 x$ per individual, no more than $40 \%$ of missing data for individual sites, and a maximum coverage of $240 x$ per individual. The cutoff for maximum coverage is $\sim 10$ times higher than the average coverage per individual, and helps to avoid SNP calls that fall in highly repetitive regions of the genome.

We used a custom pipeline to further filter the SNPs based on their position inside/outside of exons and introns. Intergenic SNPs, which did not fall within introns or exons, were used for population genomic analyses that call for neutral loci. We will refer to these as "neutral SNPs" henceforth. For analyses which included all data, including those SNPs falling within genes, we will refer to "all SNPs." To account for uncertainty in SNP and genotype calls based on allele counts, which might introduce noise or bias into downstream analyses (Johnson \& Slatkin, 2008; Lynch, 2008), we used an empirical Bayesian framework, implemented in ANGSD (analysis of next-generation sequencing data; Korneliussen, Albrechtsen \& Nielsen, 2014) to calculate genotype likelihoods instead of generating SNP genotype calls whenever possible. 


\section{Population genetic structure and demography}

Because our sampling covered a broad geographic area, it was important to quantify the underlying demographic patterns and population genetic structure before searching for genomic signatures of adaptation. To this end, we used several methods: using neutral SNPs, we performed a PCA on the genotype likelihoods, as implemented in ngsTools "PCAngsd" (Fumagalli et al., 2013). Also using the neutral SNPs, we calculated the per-individual inbreeding coefficients, as well as the pairwise relatedness of all individuals included in the study, using ngsF (Vieira et al., 2013) and ngsRelate (Korneliussen \& Moltke, 2015). Using all SNPs, we calculated the pairwise $F_{S T}$ for each sampling site in ANGSD (Fumagalli et al., 2013). We interpreted the $F_{S T}$ results using a heatmap, calculated in R (R Core Team, 2017), as well as a neighbor-joining tree calculated in the R package 'ape' (Paradis \& Schliep, 2019), visualized with FigTree (Rambaut \& Drummond, 2018).

Further, using all SNPs from a random subset of five individuals per population (to reduce run time), we calculated a phylogenetic tree with a combination of ngsDist (Vieira et al., 2016) and FastME (v.2.1.6.1) (Lefort, Desper \& Gascuel, 2015). We then looked for signatures of admixture at the individual level using ngsAdmix (Skotte, Korneliussen \& Albrechtsen, 2013). Due to the stochastic nature of simulation-based clustering programs like ngsAdmix and the like (i.e. STRUCTURE and ADMIXTURE (Pritchard, Stephens \& Donnelly, 2000; Alexander, Novembre \& Lange, 2009)), for the ngsAdmix analysis, we ran 50 replicate analyses with values of $K$ ranging from $K=2$ to $K=20$. The results of all replicates were summarized using the program CLUMPAK, which identifies the most common results across replicates for each value of $K$ (Kopelman et al., 2015). The summarized data was then used to visualize individual admixture proportions in R.

We used the results of the PCA and ngsAdmix analysis to identify larger groups within our data, for downstream genome-wide analyses that rely on pairwise comparisons, i.e. F $_{\text {ST. }}$ Running such pairwise comparisons for all sampling sites across the genome becomes difficult to interpret, while grouping at i.e. the country level is not biologically meaningful. Therefore, we used a data-informed approach and assigned the sampling sites into four groups, following the nomenclature of Krehenwinkel \& Tautz (2013): "native" (all sampling sites from France, as well as thirteen southwestern German sampling sites), "invasive" (all sampling sites from Estonia, Latvia, Lithuania, Poland, and ten northeastern German sampling sites), "Russian," and "Japanese."

\section{Genome-wide measurements}

Two common ways of detecting adaptation at the genomic level are (i) genetic differentiation outlier tests and (ii) environmental association tests (i.e. Hoban et al., 2016; Ahrens et al., 2018). In these tests, one assumes that sites which show (i) much higher than average genetic differentiation, i.e. $F_{S T}$, among populations, or (ii) high correlation with environmental variables, may play a role in local (environmental) adaptation. To identify genomic regions of interest in A. bruennichi, we used both of these methods, with an additional criterion: given the hypothesis that admixture played a role in driving the range expansion, we additionally scanned the genome for regions where the invasive/expanding populations show greater similarity (i.e. lower $F_{S T}$ ) to our Japanese populations, relative to the native/core populations. The coincidence of high environmental association with high differentiation between native and invasive populations and relatively low differentiation between invasive and Japanese populations would be taken as support for the hypothesis that introgression provided the substrate for selection in A. bruennichi. 
For the genetic differentiation outlier approach, using all SNPs, we analyzed genome-wide patterns in $\mathrm{F}_{\mathrm{ST}}$ as implemented in ANGSD (Fumagalli et al., 2013). We calculated $F_{S T}$ in sliding windows, with 50kb windows and $10 \mathrm{~kb}$ steps, with our sampling sites grouped into three populations: native, invasive and Japanese. We do not perform an explicit significance test with $\mathrm{F}_{S T}$, but look at positions with values above

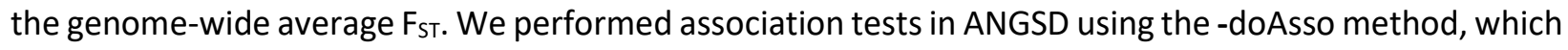
uses a generalized linear framework to associate genotype likelihoods with binary, count, or continuous variables (Skotte, Korneliussen \& Albrechtsen, 2012); in this case, we tested the association of genotypes with continuous environmental variables, namely the first five PCs from the historical climate data (see section "Historical climate data" above). This test calculates a likelihood ratio test (LRT) score, and we use a significance cutoff of 0.0005 based on a chi-square distribution, corresponding to an LRT of 12.116 as our significance threshold.

\section{Results}

\section{Common garden}

To help understand how well our models represent the data, we calculated a pseudo- $\mathrm{R}^{2}$ value. The pseudo- $R^{2}$ value can be interpreted as the amount of variation in the data, which is explained by the model. The conditional pseudo- $R^{2}$ includes random effects, while the marginal pseudo- $R^{2}$ includes only the fixed effects. A model with perfect fit to the data would have a pseudo- $R^{2}$ of 1 .

The model for clutch size had a conditional pseudo- $R^{2}$ of 0.773 and marginal pseudo- $R^{2}$ of 0.617 (Table 3 ). Spiders from the core of the range had larger clutches than spiders from the edge of the range; clutch size increased with increasing body size, and decreased with longer oviposition latency (Supplemental File 1: Figure S3A-C, Table 3). Estimated at average oviposition latency, second clutches had slightly higher clutch sizes than first clutches, with the marginal means differing by $\sim 19$ spiderlings (Supplemental File 1: Figure S3C). The hatching success model had a significant effect of origin and oviposition latency, with higher hatching success in clutches from the core of the range and with shorter latency (Supplemental File 1: Figure S3D). The effects of mother's body size and clutch (first or second) on hatching success were not significant (Table 3 ). The conditional pseudo- $R^{2}$ was 0.224 (calculating the pseudo- $R^{2}$ for betabinomial models is not straightforward, thus we do not report a marginal pseudo- $\mathrm{R}^{2}$; see methods).

Our model for overwintering survival had a pseudo- $\mathrm{R}^{2}$ of 0.262 . The interaction of winter treatment and origin showed a significant effect: under the cold winter treatment, spiderlings from edge populations survived at higher percentages than spiderlings from core populations (Figure 2A, Table 3). Survival was higher overall under the warm winter treatment (Figure 2A), and decreased over the course of winter (Figure 2B). We observed lower survival for clutches with longer oviposition latencies. Origin on its own was not significant, as it only played a role in interaction with the winter treatment. The remaining fixed effects, mother's body size and clutch, were not significant (Table 3). 
bioRxiv preprint doi: https://doi.org/10.1101/2021.11.04.467261; this version posted November 4, 2021. The copyright holder for this preprint (which was not certified by peer review) is the author/funder, who has granted bioRxiv a license to display the preprint in perpetuity. It is made available under aCC-BY-ND 4.0 International license.

Table 3: Model table for life history traits: clutch size, hatching success, and overwintering survival. Estimates are reported on the latent scale, with $95 \%$ confidence intervals. Fixed effects are considered to be significant if their confidence interval does not cross zero; these are indicated in bold text. Fixed effects separated by a colon indicate an interaction of those effects. Reference level of fixed effects is given in [square brackets].

\begin{tabular}{|c|c|c|c|c|c|c|c|}
\hline $\begin{array}{l}\text { Response } \\
\text { Variable }\end{array}$ & $\begin{array}{l}\text { Sample } \\
\text { size }\end{array}$ & $\begin{array}{l}\text { Pseudo-R } \\
\text { (cond.) }\end{array}$ & $\begin{array}{l}\text { Pseudo-R2 } \\
\text { (marg.) }\end{array}$ & Fixed effects & Estimate & $2.5 \%$ & $97.5 \%$ \\
\hline \multirow{4}{*}{$\begin{array}{l}\text { Clutch } \\
\text { size }\end{array}$} & \multirow{4}{*}{359} & \multirow[t]{4}{*}{0.773} & \multirow{4}{*}{0.617} & Origin [edge] & -0.19213 & -0.35238 & -0.03187 \\
\hline & & & & Mother's body size* & 0.20021 & 0.14292 & 0.25750 \\
\hline & & & & Clutch [2] & 0.11179 & 0.00232 & 0.22127 \\
\hline & & & & Oviposition latency* & -0.49744 & -0.55604 & -0.43884 \\
\hline \multirow{4}{*}{$\begin{array}{l}\text { Hatching } \\
\text { success }\end{array}$} & \multirow[t]{4}{*}{359} & \multirow[t]{4}{*}{0.224} & \multirow[t]{4}{*}{ NA } & Origin [edge] & -0.7730 & -1.42442 & -0.12164 \\
\hline & & & & Mother's body size* & -0.05427 & -0.31531 & 0.20678 \\
\hline & & & & Clutch [2] & 0.06178 & -0.47923 & 0.60279 \\
\hline & & & & Oviposition latency* & -0.94689 & -1.30692 & -0.58687 \\
\hline \multirow[t]{7}{*}{ Survival } & \multirow[t]{7}{*}{359} & \multirow[t]{7}{*}{0.262} & \multirow[t]{7}{*}{ NA } & Winter treatment [cold]: Origin [edge] & 0.49163 & 1.56800 & 1.03031 \\
\hline & & & & Winter treatment [cold] & -1.88981 & -1.06448 & -1.47714 \\
\hline & & & & Origin [edge] & -0.80683 & 0.16345 & -0.32169 \\
\hline & & & & Season [post-winter] & -0.76410 & -0.22459 & -0.49434 \\
\hline & & & & Clutch [2] & -0.62737 & 0.20182 & -0.21278 \\
\hline & & & & Mother's body size* & -0.18149 & 0.18805 & 0.00328 \\
\hline & & & & Oviposition latency* & -0.55753 & -0.11039 & -0.33396 \\
\hline
\end{tabular}

*centered and scaled
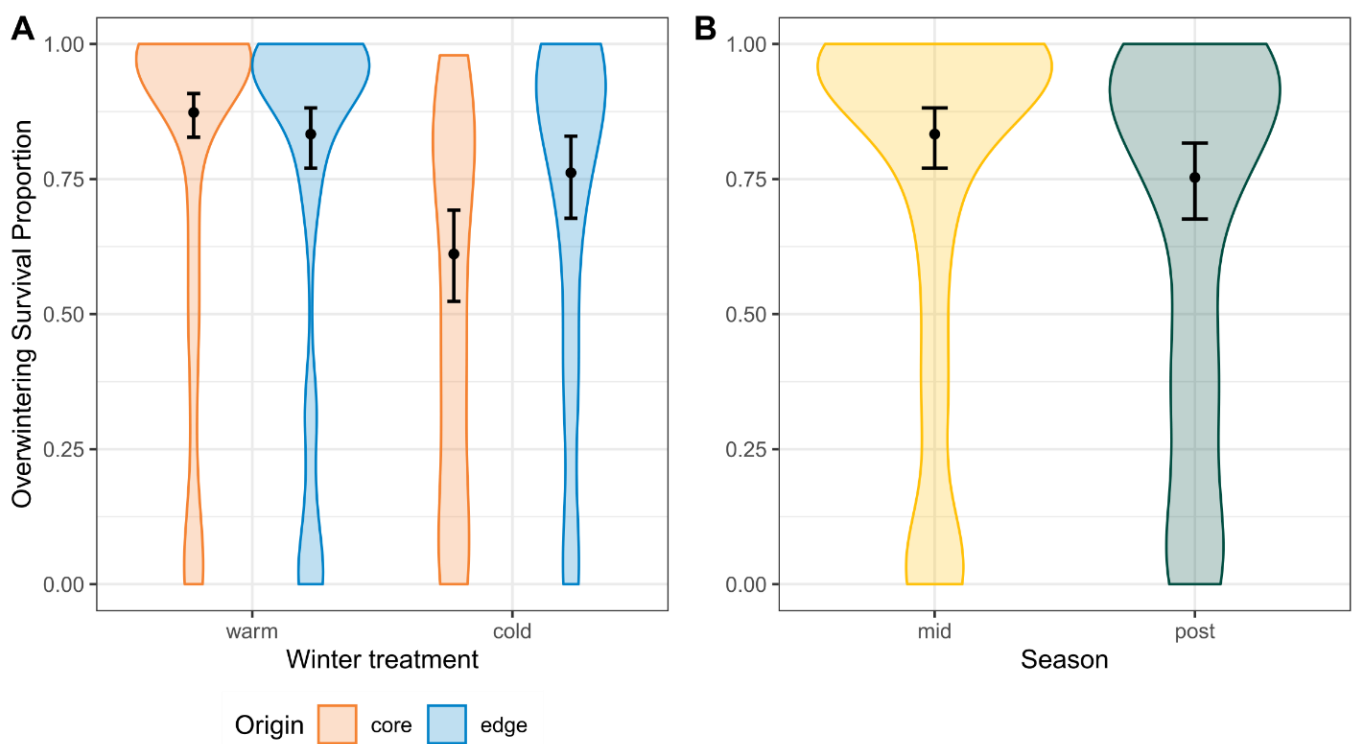

Figure 2: Overwintering survival of spiderlings according to winter treatment and origin (A) and season (B). (A) Survival according to winter treatment and origin. Under warm winter conditions, there is high survival fromboth origins. Under cold conditions, edge spiderlings have higher survival than core spiderlings. (B) Survival is higher at mid-winter and declines in post-winter. Violin plots represent the distribution of the raw data; black points with error bars represent estimated marginal means and 95\% confidence intervals for discrete predictor variables. Model predictions are back-transformed to the observed scale.

The model for lower lethal temperature had a pseudo- $R^{2}$ of 0.719 . As expected, survival decreased with decreasing exposure temperatures. The interaction of exposure temperature and origin showed that at the lowest temperatures, spiderlings from the edge of the range had higher survival than spiderlings from the core (Figure 3A). The three-way interaction of exposure temperature, winter treatment, and season 
shows that post-winter, spiderlings have a lower survival at low exposure temperatures if they have experienced a warm winter treatment (Figure 3B, Table 4).
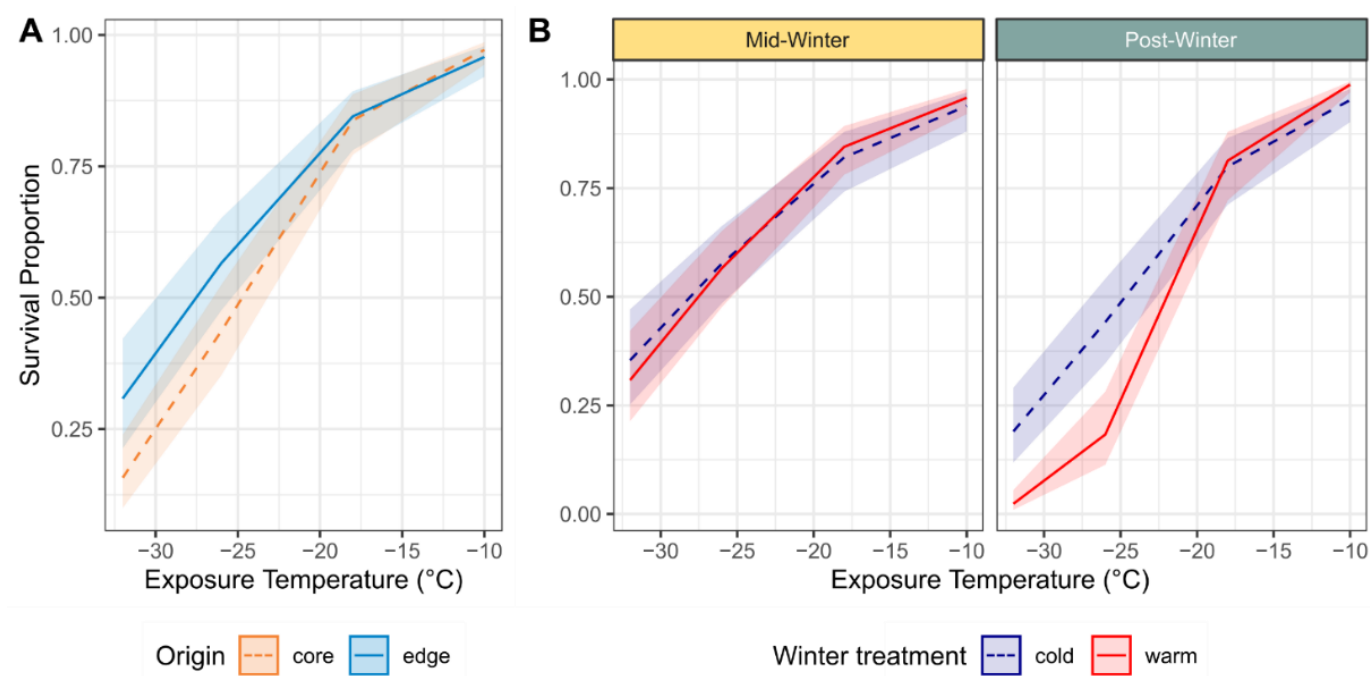

Figure 3: Lower lethal temperature according to $(A)$ origin and exposure temperature and (B) the interaction of exposure temperature with season (mid- or post-winter) and warm or cold winter treatments. Lines represent model predictions flanked with their 95\% confidence intervals. Model predictions are back-transformed to the observed scale. (A) Regardless of experimental treatment, edge spiderlings have higher survival at the coldest exposure temperatures. (B) At mid-winter (left), there is no difference in survival between warm and cold winter treatments. Post-winter (right), survival at the lowest exposure temperatures decreases dramatically for spiderlings in the warm winter treatment.

We analyzed the data on supercooling points in two models, due to multimodality of the raw data: firstly, we analyzed the "low group" (LG) SCPs as a continuous variable, measured at the spiderling level. SCPS were classified into the $L G$ if they were below $-25.5^{\circ} \mathrm{C}$. Secondly, we analyzed the per-clutch ratio of $L G$ to "high group" (HG) SCPs (SCPs higher than $-25-5^{\circ} \mathrm{C}$, see methods and Supplemental File 1: Figure S2). For the LG SCPs, our had a conditional pseudo- $R^{2}$ of 0.301 and a marginal pseudo- $R^{2}$ of 0.245 . This relatively low $R^{2}$ may be due to the arbitrary splitting of the data into groups. Overall, spiderlings from the edge populations had lower SCPs than spiderlings from the core (Figure 4A). The SCP increased post-winter, and was notably higher in the post-winter warm treatment Table 4. The model comparing the ratio of LG:HG SCPs had a pseudo- $R^{2}$ of 0.841 . Post-winter, across all treatments, there is a shift toward more HG SCPs. For egg sacs from the core of the range, post-winter, under a warm winter treatment, the ratio dips strongly toward more HG SCPs (Figure 4B, Table 4). 
A Low Group Supercooling Points

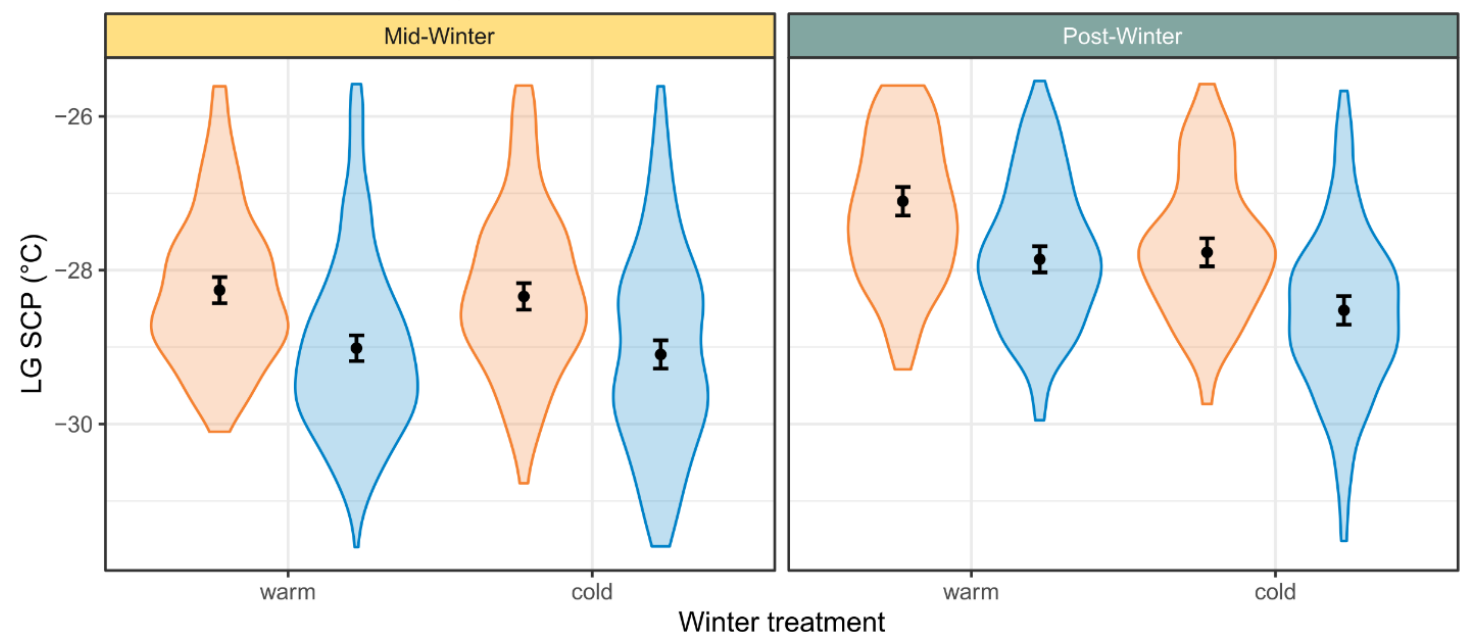

B Proportion of LG:HG Supercooling Points

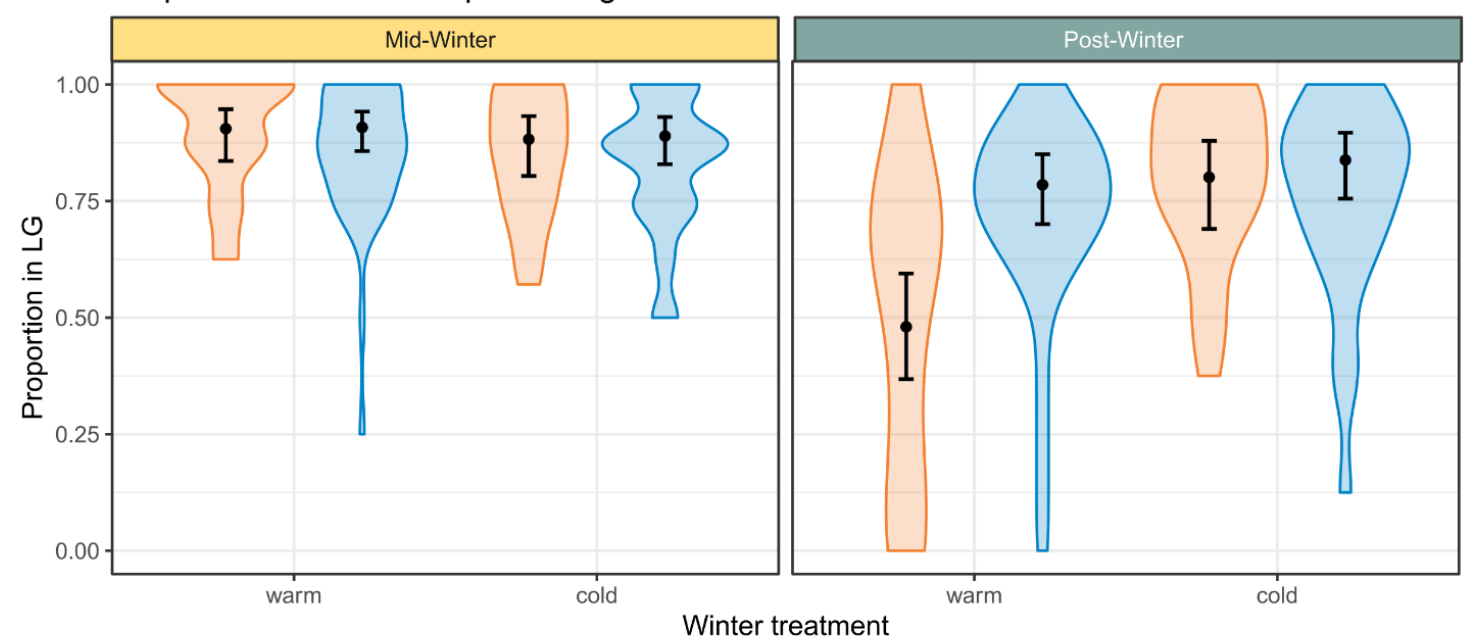

Origin $\square$ core $\square$ edge

Figure 4. Supercooling points according to winter treatment, origin and season. "Low group" (LG) supercooling points are plotted in (A), while the ratio (see methods) of LG:HG ("high group") supercooling points is plotted in (B). Violin plots represent the distribution of the raw data; black points with error bars represent estimated marginal means and $95 \%$ confidence intervals for discrete predictor variables. Model predictions are back-transformed to the observed scale.

As the last cold-tolerance-related trait, we analyzed the chill coma recovery time of spiderlings, in response to the interaction of winter temperature, origin and season, and the two-way interactions of those fixed effects, along with clutch, mother's leg length, fecundity of the egg sac, and oviposition latency. The conditional pseudo- $R^{2}$ was 0.010 , and the marginal pseudo- $R^{2}$ was 0.002 . Nearly all effects were significant, but given the extremely low $R^{2}$ values, we will not interpret the results of the model. Estimates and confidence intervals are nonetheless presented in Table 4. 
bioRxiv preprint doi: https://doi.org/10.1101/2021.11.04.467261; this version posted November 4, 2021. The copyright holder for this preprint (which was not certified by peer review) is the author/funder, who has granted bioRxiv a license to display the preprint in perpetuity. It is made available under aCC-BY-ND 4.0 International license.

Table 4: Model table for cold-tolerance-related traits in offspring. Estimates are reported on the latent scale, with $95 \%$ confidence intervals. Fixed effects are considered to be significant if their confidence interval does not cross zero; these are indicated in bold text. Fixed effects separated by a colon indicate an interaction of those effects. Reference level of fixed effects is given in [square brackets].

\begin{tabular}{|c|c|c|c|c|c|c|c|}
\hline $\begin{array}{l}\text { Response } \\
\text { Variable }\end{array}$ & $\begin{array}{l}\text { Sample } \\
\underline{\text { size }}\end{array}$ & $\frac{\text { Pseudo-R }}{\text { (cond.) }}$ & $\frac{\text { Pseudo- } R^{2}}{\text { (marg.) }}$ & $\underline{\text { Fixed effects }}$ & $\underline{2.5 \%}$ & $\underline{97.5 \%}$ & Estimate \\
\hline \multirow{11}{*}{$\begin{array}{l}\text { Lower lethal } \\
\text { temperature } \\
\text { survival }\end{array}$} & \multirow[t]{11}{*}{371} & \multirow[t]{11}{*}{0.719} & \multirow[t]{11}{*}{ NA } & $\begin{array}{l}\text { Exposure temperature: Winter treatment } \\
\text { [warm]: season [post-winter] }\end{array}$ & 0.04335 & 0.239335 & 0.14134 \\
\hline & & & & Exposure temperature: Origin [edge] & -0.10268 & -0.01348 & -0.05807 \\
\hline & & & & $\begin{array}{l}\text { Winter treatment [warm]: season [post- } \\
\text { winter] }\end{array}$ & 0.17541 & 4.73690 & 2.45615 \\
\hline & & & & Exposure temperature: season [post-winter] & -0.00914 & 0.11022 & 0.05054 \\
\hline & & & & $\begin{array}{l}\text { Exposure temperature: winter treatment } \\
\text { [warm] }\end{array}$ & -0.02695 & 0.08140 & 0.02722 \\
\hline & & & & Oviposition latency* & -0.46991 & 0.00162 & -0.23415 \\
\hline & & & & Clutch [2] & -0.70801 & 0.24224 & -0.23289 \\
\hline & & & & Origin [edge] & -2.11262 & 0.13284 & -0.98989 \\
\hline & & & & Season [post-winter] & -0.70449 & 2.24726 & 0.77138 \\
\hline & & & & Winter treatment [warm] & -0.66658 & 1.99523 & 0.66433 \\
\hline & & & & Exposure temperature & 0.16124 & 0.25850 & 0.20987 \\
\hline \multirow{8}{*}{$\begin{array}{l}\text { Low-group } \\
\text { supercooling } \\
\text { point }\end{array}$} & \multirow[t]{8}{*}{1493} & \multirow[t]{8}{*}{0.301} & \multirow[t]{8}{*}{0.245} & $\begin{array}{l}\text { Winter treatment warm: season [post- } \\
\text { winter] }\end{array}$ & 0.31763 & 0.84627 & 0.58195 \\
\hline & & & & Oviposition latency* & -0.24258 & -0.00054 & -0.12156 \\
\hline & & & & Mother's body size* & -0.03738 & 0.16173 & 0.06218 \\
\hline & & & & Clutch size* & -0.08836 & 0.11005 & 0.01085 \\
\hline & & & & Clutch [2] & -0.13729 & 0.28251 & 0.07261 \\
\hline & & & & Origin [edge] & -0.96994 & -0.53823 & -0.75409 \\
\hline & & & & Season [post-winter] & 0.37844 & 0.76854 & 0.57349 \\
\hline & & & & Winter treatment [warm] & -0.09594 & 0.25923 & 0.08164 \\
\hline \multirow{11}{*}{$\begin{array}{l}\text { Ratio of low- } \\
\text { group to } \\
\text { high-group } \\
\text { supercooling } \\
\text { points }\end{array}$} & \multirow[t]{11}{*}{245} & \multirow[t]{11}{*}{0.841} & \multirow[t]{11}{*}{ NA } & $\begin{array}{l}\text { Winter treatment [warm]: Origin [edge]: } \\
\text { Season [post-winter] }\end{array}$ & -0.19075 & 2.51019 & 1.15972 \\
\hline & & & & Origin [edge]: Season [post-winter] & -0.81417 & 1.17615 & 0.18099 \\
\hline & & & & $\begin{array}{l}\text { Winter treatment [warm]: Season [post- } \\
\text { winter] }\end{array}$ & -2.78383 & -0.64061 & -1.71222 \\
\hline & & & & Winter treatment [warm]: Origin [edge] & -1.04706 & 0.97327 & -0.03690 \\
\hline & & & & Oviposition latency* & 0.14192 & 0.75032 & 0.44612 \\
\hline & & & & Mother's body size* & -0.19871 & 0.29809 & 0.04969 \\
\hline & & & & Clutch size* & -0.30033 & 0.14671 & -0.07681 \\
\hline & & & & Clutch [2] & -0.92664 & 0.14684 & -0.38990 \\
\hline & & & & Season [post-winter] & -1.41845 & 0.17110 & -0.62367 \\
\hline & & & & Origin [edge] & -0.73061 & 0.86961 & 0.06950 \\
\hline & & & & Winter treatment [warm] & -0.58626 & 1.06673 & 0.24023 \\
\hline \multirow{11}{*}{$\begin{array}{l}\text { Chill coma } \\
\text { recovery } \\
\text { time }\end{array}$} & \multirow[t]{11}{*}{2072} & \multirow[t]{11}{*}{0.010} & \multirow[t]{11}{*}{0.002} & $\begin{array}{l}\text { Winter treatment [warm]: Origin [edge]: } \\
\text { Season [post-winter] }\end{array}$ & -0.17466 & -0.03670 & -0.10568 \\
\hline & & & & Origin [edge]: Season [post-winter] & 0.01149 & 0.11814 & 0.06482 \\
\hline & & & & $\begin{array}{l}\text { Winter treatment [warm]: Season [post- } \\
\text { winter] }\end{array}$ & 0.06088 & 0.16448 & 0.11268 \\
\hline & & & & Winter treatment [warm]: Origin [edge] & -0.00049 & 0.09734 & 0.04842 \\
\hline & & & & Oviposition latency* & 0.01005 & 0.03846 & 0.02425 \\
\hline & & & & Mother's body size* & -0.02633 & -0.00047 & -0.01340 \\
\hline & & & & Clutch size* & 0.00579 & 0.03019 & 0.01799 \\
\hline & & & & Clutch [2] & -0.05448 & -0.00392 & -0.02920 \\
\hline & & & & Season [post-winter] & -0.12642 & -0.04683 & -0.08662 \\
\hline & & & & Origin [edge] & -0.06334 & 0.02267 & -0.02033 \\
\hline & & & & Winter treatment [warm] & -0.09988 & -0.02591 & -0.06289 \\
\hline
\end{tabular}

*centered and scaled 
The conditional pseudo- $R^{2}$ for the offspring mass model was 0.794 , with a marginal pseudo- $R^{2}$ of 0.307 (Table 5). Core offspring had higher mass than edge offspring irrespective of season or treatment, and the warm winter treatment resulted in lower mass overall. Mass decreased over the course of winter (Figure 5A). In egg sacs with higher clutch sizes, offspring mass was slightly lower. Additionally, offspring from later-laid egg sacs had lower body mass, while second clutches had higher body mass (Table 5).

For the leg length model, we could not include the sampling population as a random effect because of singular fit, due to the very high variance explained by mother's ID and egg sac. The model had a conditional pseudo- $\mathrm{R}^{2}$ of 0.817 , with a marginal pseudo- $\mathrm{R}^{2}$ of only 0.060 (Table 5). Evidently, offspring body size has a very strong maternal component. Offspring from the edge of the range are smaller than offspring from the core of the range (Figure 5B)
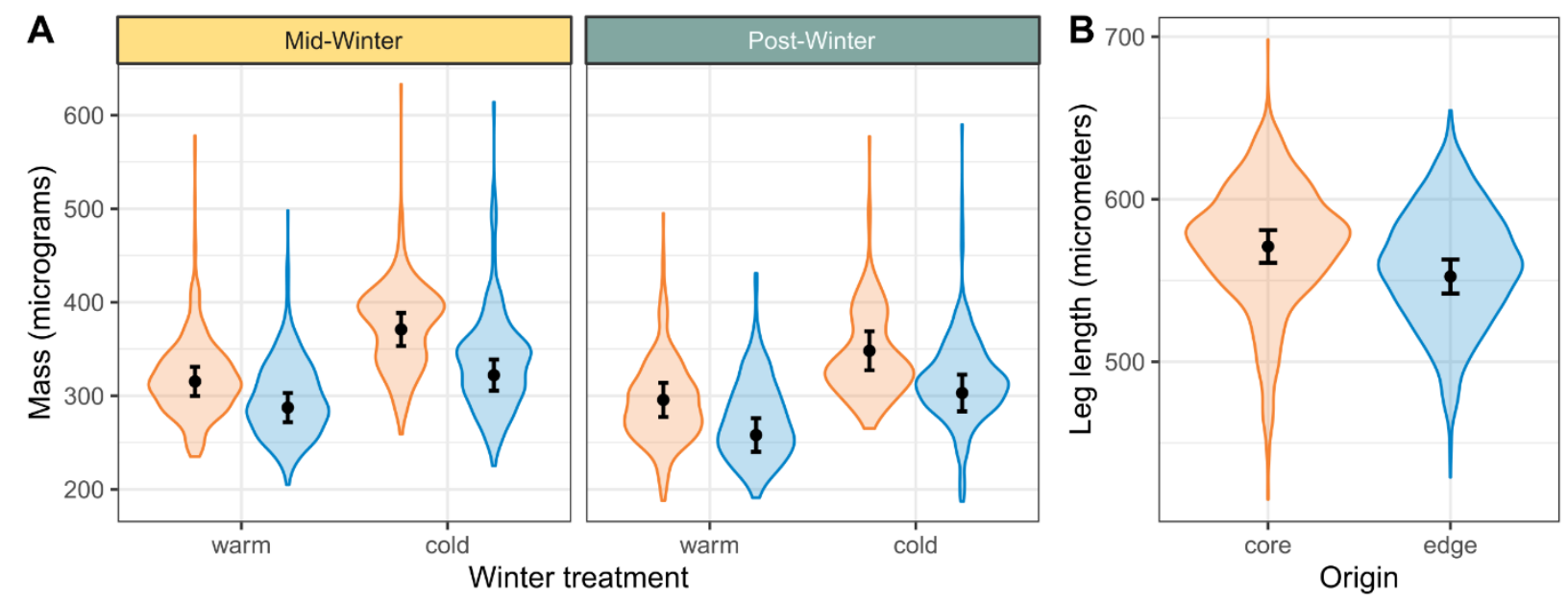

Origin $\square$ core $\square$ edge

Figure 5. Body size of offspring according to winter treatment and origin. (A) Body mass decreased from mid- to post-winter, and is lower following a warm winter treatment. (B) Spiderlings from the core of the range are larger than those from the edge of the range. Transparent points represent the raw data; black points with error bars represent estimated marginal means and 95\% confidence intervals for discrete predictor variables. Model predictions are back-transformed to the observed scale.

Table 5. Model table for offspring body size. Estimates are reported on the latent scale, with $95 \%$ confidence intervals. Fixed effects are considered to be significant if their confidence interval does not cross zero; these are indicated in bold text. Fixed effects separated by a colon indicate an interaction of those effects. Reference level of fixed effects is given in [square brackets].

\begin{tabular}{|c|c|c|c|c|c|c|c|}
\hline$\frac{\text { Response }}{\underline{\text { Variable }}}$ & $\begin{array}{l}\text { Sample } \\
\underline{\text { size }}\end{array}$ & $\begin{array}{l}\text { Pseudo-R } R^{2} \\
\text { (cond.) }\end{array}$ & $\begin{array}{l}\frac{\text { Pseudo- } R^{2}}{\text { (marg.) }} \\
\end{array}$ & $\underline{\text { Fixed effects }}$ & $\underline{2.5 \%}$ & $\underline{97.5 \%}$ & Estimate \\
\hline \multirow[t]{7}{*}{$\begin{array}{l}\text { Offspring } \\
\text { mass }\end{array}$} & 3539 & 0.794 & 0.307 & $\begin{array}{l}\text { Winter treatment [warm]: Origin } \\
\text { [edge]: Season [post-winter] }\end{array}$ & -51.08397 & 26.81681 & -13.28800 \\
\hline & & & & Origin [edge]: Season [post-winter] & -21.82160 & 36.49953 & 3.67300 \\
\hline & & & & $\begin{array}{l}\text { Winter treatment [warm]: Season } \\
\text { [post-winter] }\end{array}$ & -26.18663 & 32.26970 & 3.01500 \\
\hline & & & & $\begin{array}{l}\text { Winter treatment [warm]: Origin } \\
\text { [edge] }\end{array}$ & -6.92649 & 44.57374 & 20.85400 \\
\hline & & & & Winter treatment [warm] & -74.86328 & -35.33104 & -55.53500 \\
\hline & & & & Origin [edge] & -67.48434 & -22.59316 & -48.81600 \\
\hline & & & & Season [post-winter] & -45.91207 & -1.31285 & -22.76000 \\
\hline
\end{tabular}




\begin{tabular}{|c|c|c|c|c|c|c|c|}
\hline & & & & Clutch [2] & 5.51927 & 35.04812 & 19.82900 \\
\hline & & & & Clutch size* & -13.72314 & -0.23715 & -6.72100 \\
\hline & & & & Mother's body size* & -0.55694 & 14.51010 & 5.76000 \\
\hline & & & & Oviposition latency* & -22.97329 & -3.05954 & -12.74300 \\
\hline \multirow[t]{11}{*}{$\begin{array}{l}\text { Offspring } \\
\text { body size }\end{array}$} & \multirow[t]{11}{*}{3539} & \multirow[t]{11}{*}{0.817} & \multirow[t]{11}{*}{0.060} & $\begin{array}{l}\text { Winter treatment [warm]: Origin } \\
\text { [edge]: Season [post-winter] }\end{array}$ & -43.8826 & 25.60044 & -9.2694 \\
\hline & & & & Origin [edge]: Season [post-winter] & -29.1719 & 22.78383 & -3.1507 \\
\hline & & & & $\begin{array}{l}\text { Winter treatment [warm]: Season } \\
\text { [post-winter] }\end{array}$ & -12.98791 & 39.09495 & 13.1046 \\
\hline & & & & $\begin{array}{l}\text { Winter treatment [warm]: Origin } \\
\text { [edge] }\end{array}$ & -5.73829 & 39.98230 & 17.1617 \\
\hline & & & & Winter treatment [warm] & -32.89174 & 2.32406 & -15.2201 \\
\hline & & & & Origin [edge] & -45.18832 & -5.20747 & -25.2248 \\
\hline & & & & Season [post-winter] & -22.01804 & 17.68051 & -2.2011 \\
\hline & & & & Clutch [2] & -14.16640 & 12.25400 & -0.953 \\
\hline & & & & Clutch size* & -5.74777 & 7.69614 & 0.9909 \\
\hline & & & & Mother's body size* & -10.76298 & 1.28542 & -4.7659 \\
\hline & & & & Oviposition latency* & -18.65102 & -0.88816 & -9.8212 \\
\hline
\end{tabular}

*centered and scaled

\section{Phenotype-environment associations across transect}

We characterized the environmental variation across our sampling sites using PCA. The first five environmental PCs accounted for $97.6 \%$ of the variation in the climate data. PC1 alone accounted for $51.9 \%$ of the variation, and varies almost perfectly with latitude (Supplemental File 1: Figure S4). The strongest loadings of this PC were temperature-related, such as the mean temperature of the coldest quarter, the minimum temperature of the coldest month, annual mean temperature, isothermality, and temperature seasonality, although almost all environmental variables had strong contributions to this PC. PC2 accounted for $24.4 \%$ of variation, and separated sites more according to precipitation than temperature, with the strongest loadings being the precipitation of the warmest quarter, precipitation of the wettest quarter, annual precipitation, and precipitation of the driest month. PC3 accounted for $11.2 \%$ of variation, with the strongest loadings relating to seasonality in temperature and precipitation, as well as the annual temperature range. PC4, which accounted for $6.7 \%$ of variation, was strongly related to the mean temperature of the wettest quarter. The strongest loadings for PC5, which accounted for $3.4 \%$ of environmental variation, were similar to $\mathrm{PC} 3$, but temperature and seasonal precipitation contributed in opposite directions for this PC. See Supplemental File 3 for PCA biplots and loadings of each PC.

Using those 5 PCs, we assessed how adult female traits (leg length, opisthosoma pigmentation, fecundity, and hatching success) vary with the environment. Sampling site was always included as a random effect. Female fecundity and the hatching success of their egg sacs had no significant associations with environmental PCs. Female leg length, however, had a significant association with PC1: leg length is shorter, i.e. spiders mature at a smaller body size, in environments characterized by lower temperatures and greater seasonality. Opisthosoma pigmentation had a significant relationship with PC4, but did not vary with latitude (i.e. PC1) (Figure 6, Table 6). 
bioRxiv preprint doi: https://doi.org/10.1101/2021.11.04.467261; this version posted November 4, 2021. The copyright holder for this preprint (which was not certified by peer review) is the author/funder, who has granted bioRxiv a license to display the preprint in perpetuity. It is made available under aCC-BY-ND 4.0 International license.

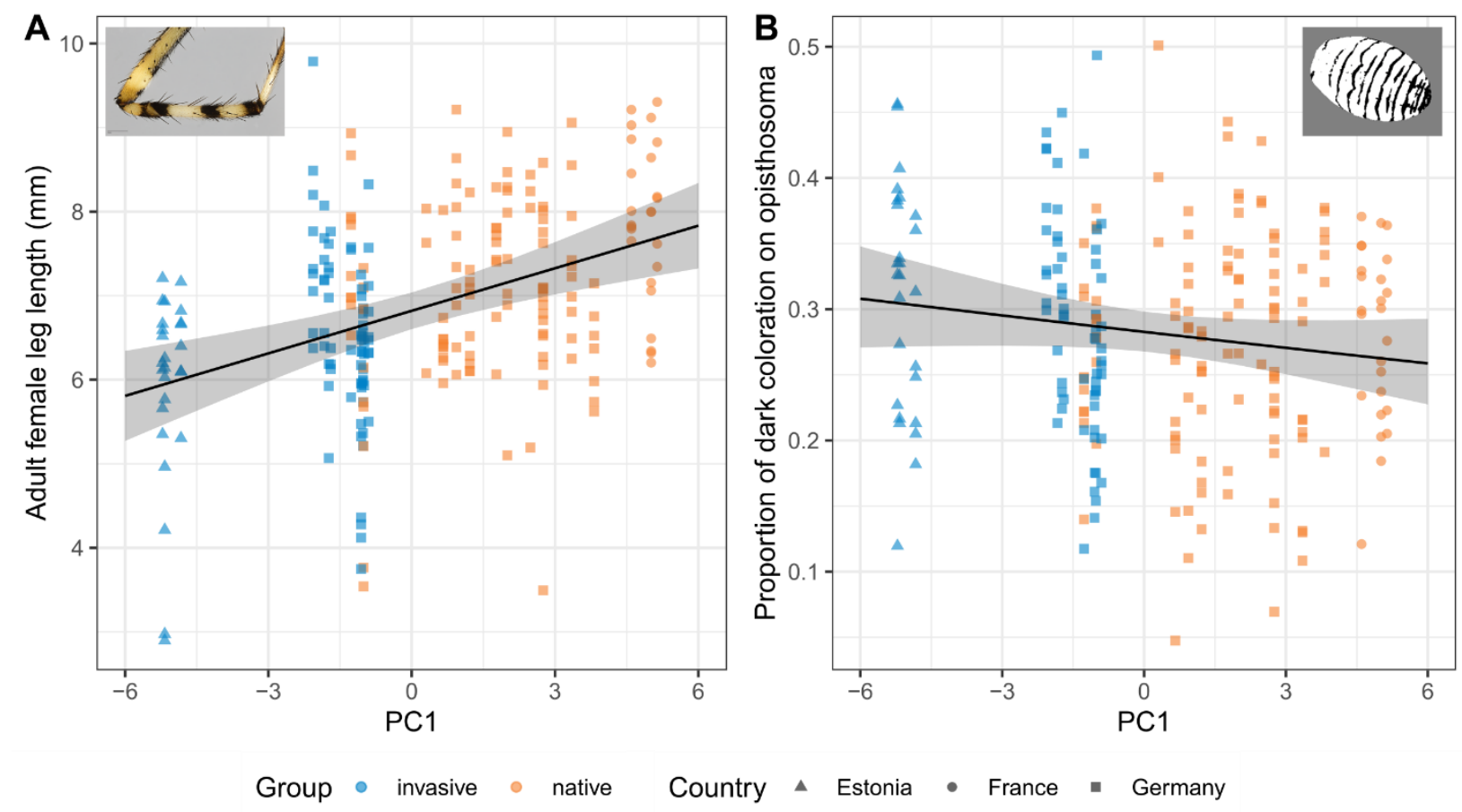

Figure 6: Female traits according to the environment. Points are colored according to their assignment to "invasive" or "native" genetic clusters (see population genetic results, i.e. Figure 7, below), and shaped according to the sampling country. (A) Adult female tibia-patella length, a body size proxy, increases with environmental PC1, i.e. we observe larger body sizes under the warm temperatures at lower latitudes. (B) Opisthosoma pigmentation does not vary according to PC1.

Table 6. Model table for adult female traits according to environmental principle components. Estimates are reported on the latent scale, with $95 \%$ confidence intervals. Fixed effects are considered to be significant if their confidence interval does not cross zero; these are indicated in bold text.

\begin{tabular}{|c|c|c|c|c|c|c|c|}
\hline Response Variable & $\underline{\text { Sample size }}$ & Pseudo- $R^{2}$ (cond.) & Pseudo- $\mathrm{R}^{2}$ (marg.) & $\underline{\text { Fixed effects }}$ & $\underline{2.5 \%}$ & $\underline{97.5 \%}$ & Estimate \\
\hline \multirow[t]{5}{*}{ Adult female body size } & \multirow[t]{5}{*}{273} & \multirow[t]{5}{*}{0.419} & \multirow[t]{5}{*}{0.187} & PC1 & 87.21212 & 250.541 & 168.93 \\
\hline & & & & PC2 & -58.018 & 161.5652 & 51.46 \\
\hline & & & & PC3 & -260.58 & 192.3862 & -33.77 \\
\hline & & & & PC4 & -44.8432 & 378.1135 & 167.04 \\
\hline & & & & PC5 & -226.937 & 496.4214 & 133.94 \\
\hline \multirow[t]{5}{*}{ Opisthosoma pigmentation } & \multirow[t]{5}{*}{262} & \multirow[t]{5}{*}{0.280} & \multirow[t]{5}{*}{ NA } & PC1 & -0.04675 & 0.006182 & -0.02029 \\
\hline & & & & PC2 & -0.06909 & 0.003372 & -0.03286 \\
\hline & & & & PC3 & -0.06669 & 0.08155 & 0.007431 \\
\hline & & & & PC4 & 0.026992 & 0.164237 & 0.095615 \\
\hline & & & & PC5 & -0.01846 & 0.215921 & 0.098732 \\
\hline \multirow[t]{5}{*}{ Clutch size } & \multirow[t]{5}{*}{273} & \multirow[t]{5}{*}{0.323} & \multirow[t]{5}{*}{0.012} & PC1 & -0.05979 & 0.033122 & -0.01333 \\
\hline & & & & PC2 & -0.08344 & 0.039135 & -0.02215 \\
\hline & & & & PC3 & -0.11297 & 0.14434 & 0.015687 \\
\hline & & & & PC4 & -0.08279 & 0.155782 & 0.036496 \\
\hline & & & & PC5 & -0.18293 & 0.227001 & 0.022036 \\
\hline \multirow[t]{5}{*}{ Hatching success } & \multirow[t]{5}{*}{273} & \multirow[t]{5}{*}{0.009} & \multirow[t]{5}{*}{ NA } & PC1 & -0.12185 & 0.033268 & -0.04429 \\
\hline & & & & PC2 & -0.05821 & 0.147993 & 0.04489 \\
\hline & & & & PC3 & -0.23168 & 0.205299 & -0.01319 \\
\hline & & & & PC4 & -0.34267 & 0.045613 & -0.14853 \\
\hline & & & & PC5 & -0.63409 & 0.058033 & -0.28803 \\
\hline
\end{tabular}

*centered and scaled 


\section{Genomic data}

\section{SNP calling and filtering}

After demultiplexing and trimming adapters, the ddRAD libraries for 423 adult females yielded more than 362 million reads, with an average of 815,796 reads per sample (min: 5,296 reads, max: 12,597,258 reads). An average (mean) of $86.89 \%$ of reads per sample mapped to the reference genome, with a mean coverage of 4.94X for each of the 13 chromosome-level superscaffolds (Sheffer et al., 2021b). SNP calling resulted in 22,372 biallelic SNPs. We then filtered the SNPs according to their position within exons and introns: 13,832 "neutral" SNPs did not fall within exons or introns.

\section{Population genetic structure and demography}

We performed several analyses to understand the underlying population structure and demographic processes in A. bruennichi. Firstly, we used the neutral SNP subset to perform a principal components analysis (PCA) using PCAngsd (Meisner \& Albrechtsen, 2018), with three subsets of the samples: (i) all populations, including Japan; (ii) all populations, excluding Japan; and (iii) all European populations, excluding Japan and Russia, as the main focus of our study is the transect through Europe. Results for the PCA including Japan, and the PCA excluding Japan but including Russia, can be found in the supplementary material (Supplemental File 1: Figure S5, Figure S6). In the Europe-only PCA, PC1 accounted for $17.4 \%$ of genetic variation, separating French and southwestern German populations from the northeastern European populations, while PC2 accounted for $3.4 \%$ of the variation, separating Estonian populations from the remaining European populations (Figure 7).

Using ngsAdmix (Skotte et al., 2013), we calculated individual admixture proportions, with values of $K$ ranging from $K=2$ to $K=20$. Plots for $K=4-8$ can be found in the supplementary material (Supplemental File 1: Figure S7); here, we present the results for $K=2$, to correspond with the previous STRUCTURE analysis from Krehenwinkel \& Tautz (2013), and $K=3$, which best shows the transition between native and invasive populations (Figure 7B). In the analysis with $K$ set to 2, we find, as in Krehenwinkel \& Tautz (2013), that the proportion of assignment to the "Japanese" cluster gradually increases with latitude, and is highest in Russian populations. The $K=3$ results show a rapid turnover within Germany: in southwestern Germany, individuals are assigned to the "native" genetic cluster, and in the center of Germany, there is turnover to individuals being assigned to the "invasive" cluster. The turnover occurs between populations 20 and 21 , which are just 85 kilometers apart. 
bioRxiv preprint doi: https://doi.org/10.1101/2021.11.04.467261; this version posted November 4, 2021. The copyright holder for this preprint (which was not certified by peer review) is the author/funder, who has granted bioRxiv a license to display the preprint in perpetuity. It is made available under aCC-BY-ND 4.0 International license.
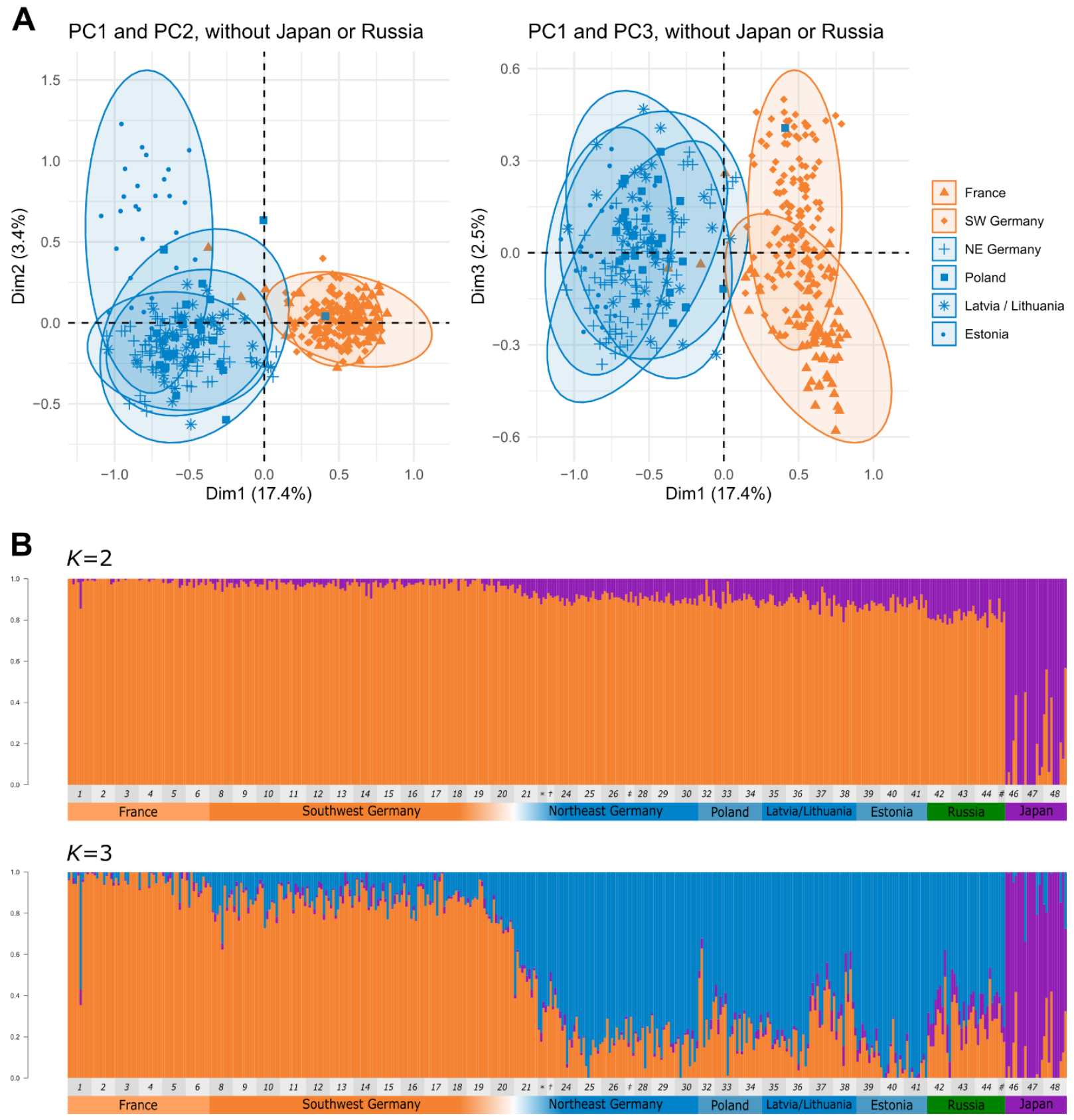

Figure 7. Population genetic structure. (A) Principal components analysis of European populations using neutral SNPs. Ellipses represent 95\% confidence intervals; points represent individual samples, with shape indicating the sampling country. Points and ellipses are colored according to the site's "native" (orange) or "invasive" (blue) assignment. (B) Stacked barplots of individual ancestry assignment, using ngsAdmix results for $K=2$ and $K=3$. Numbers below the bars show the sampling sites. For small sampling sites where a number did not fit, * represents population number 22, † represents 23 , ¥ represents 27 , and \# represents 45 .

We calculated the levels of inbreeding at every sampling site, to gain insight into the levels of isolation of our populations. The Japanese populations showed high levels of inbreeding, while the remaining populations were mostly outbreeding, especially the Russian populations (Figure 8A). Interestingly, the populations at the edge of the range in Estonia (population numbers 39, 40, and 41) show a linear increase in the level of inbreeding with latitude. 
bioRxiv preprint doi: https://doi.org/10.1101/2021.11.04.467261; this version posted November 4, 2021. The copyright holder for this preprint (which was not certified by peer review) is the author/funder, who has granted bioRxiv a license to display the preprint in perpetuity. It is made available under aCC-BY-ND 4.0 International license.

We also calculated the pairwise $F_{S T}$ between all sampling sites, which ranged from 0.018 between two Russian sampling sites to 0.358 between a site in Japan and a site in southern France. As in the PCA, the highest differentiation was found between Japanese and all other populations (Supplemental File 1: Table S1). Within the European (native and invasive) populations, $\mathrm{F}_{S T}$ was quite low, ranging from 0.025-0.130. FST was lower among "native" populations (max: 0.074) than among "invasive" populations (max: 0.130) (Figure 8B), and $\mathrm{F}_{\text {ST }}$ was very low among Russian populations (max: 0.036) (Supplemental File 1: Table S1). As an additional visualization, we used the pairwise $F_{S T}$ matrix to calculate a neighbor-joining tree in the $R$ package 'ape' (Paradis \& Schliep, 2019), which showed the native populations in one cluster, while the invasive populations did not cluster as clearly, and were more similar to the Russian and Japanese populations (Figure 8C).

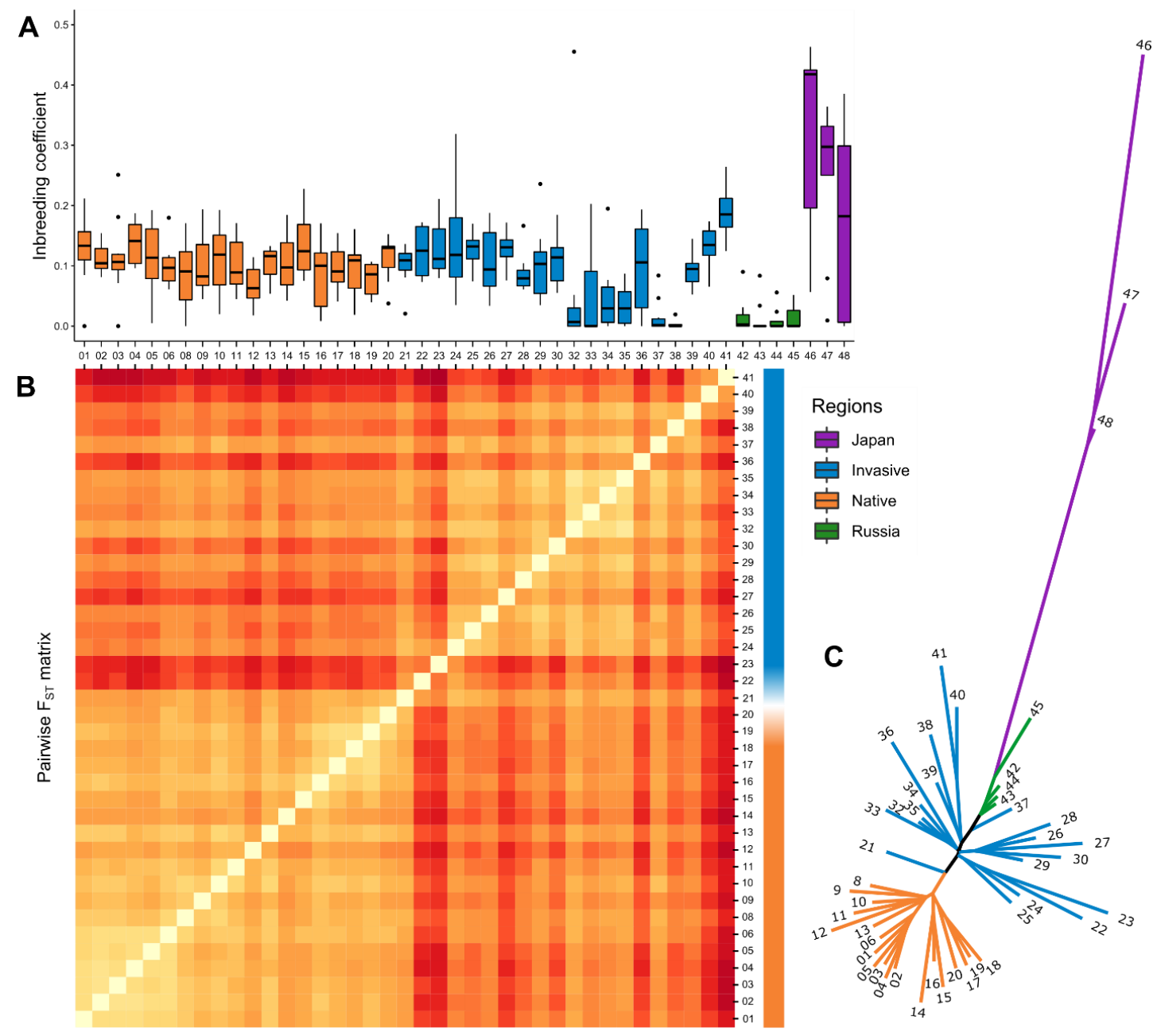

Figure 8. Demography and differentiation. (A) Boxplots of individual inbreeding coefficients for every sampling site. Boxes encompass the $1^{\text {st }}$ and $3^{\text {rd }}$ quartiles of the data and are colored according to sampling region. Thick horizontal lines represent the median, thin vertical lines represent 1.5 times the upper and lower interquartile range, and black points represent outliers. (B) Symmetrical heat-map of pairwise Fst between all European populations. The color scale runs from light yellow (lowest differentiation) to dark red (highest differentiation). Color blocks on the right side of the heat-map represent the groupings of populations into "native" and "invasive" clusters. (C) Neighborjoining tree of FST matrix. Branch labels represent the population numbers, and branches are colored by their regional groupings. In (A) and (B), populations are ordered in the same way, to directly compare between the two different statistics. 
We constructed another phylogenetic tree using ngsDist (Vieira et al., 2016) and FastME (v.2.1.6.1) (Lefort et al., 2015), which revealed the same large-scale groupings of native, invasive, Russian and Japanese populations as the PCA, admixture, and $\mathrm{F}_{\mathrm{ST}}$ analyses (Supplemental File 1: Figure S8). Bootstrap support was high for the splits between the Japanese, Russian, and European clades, but was quite low within Europe.

\section{Genome-wide evidence for environmental adaptation}

We analyzed genome-wide patterns of genetic differentiation ( $\left.F_{S T}\right)$ and genotype-environment associations to identify potential regions of adaptive introgression. Using the output of both tests, we searched for genomic regions where $F_{\text {ST }}$ between native and invasive populations was particularly high, association with the environment was strong, and $\mathrm{F}_{\mathrm{ST}}$ between invasive and Japanese sites was relatively low. We will interpret this pattern as a signature of adaptive introgression.

Firstly, we looked at associations with environmental PC1, which represents the environmental variables that vary with latitude, mostly relating to cold temperatures. 589 SNPs had significant associations with this latitudinal environmental variation. We found windows showing signatures of adaptive introgression on almost all chromosomes, with particularly strong signatures on chromosome 9. Two regions on chromosome 9, centered at around $29 \mathrm{Mbp}$ and $58 \mathrm{Mbp}$, show exactly the pattern we would expect for adaptive introgression: high association with the environment, high differentiation within Europe, and lower differentiation between invasive and Japanese populations. There is another region on chromosome 9, centered at around $107 \mathrm{Mbp}$, which shows very high environmental association and high differentiation within Europe, but the last criterion of similarity between invasive and Japanese populations is less striking in this region. We also found a very interesting region on chromosome 2; while the range of values on this chromosome was lower, there is an approximately $30 \mathrm{Mbp}$-long region showing a possible signature of adaptive introgression (Figure 10). Boxplots of significant LRT values per chromosome can be found in Supplemental File 1: Figure S10.

For the other environmental principal components, PC2-5, we found relatively few significant associations: 84 for PC2, 186 for PC3, 53 for PC4, and 109 for PC5. For PC2 and PC4, there were no characteristic patterns of adaptive introgression, and indeed few areas where outliers were found between both the environmental associations and $\mathrm{F}_{\mathrm{ST}}$. Environmental PCS 2 and 4 are characterized by variables related to precipitation. On the other hand, environmental PC3 is driven by seasonality in temperature and precipitation, and in that case, we see more significant associations, and more signatures of adaptive introgression. Similarly, PC5 is also related to seasonality, in a slightly different way, as it differentiates between precipitation seasonality and temperature seasonality. The genomic regions displaying signatures of adaptive introgression for PC3 and PC5 are the same as those for PC1. 
bioRxiv preprint doi: https://doi.org/10.1101/2021.11.04.467261; this version posted November 4, 2021. The copyright holder for this preprint (which was not certified by peer review) is the author/funder, who has granted bioRxiv a license to display the preprint in perpetuity. It is made available under aCC-BY-ND 4.0 International license.
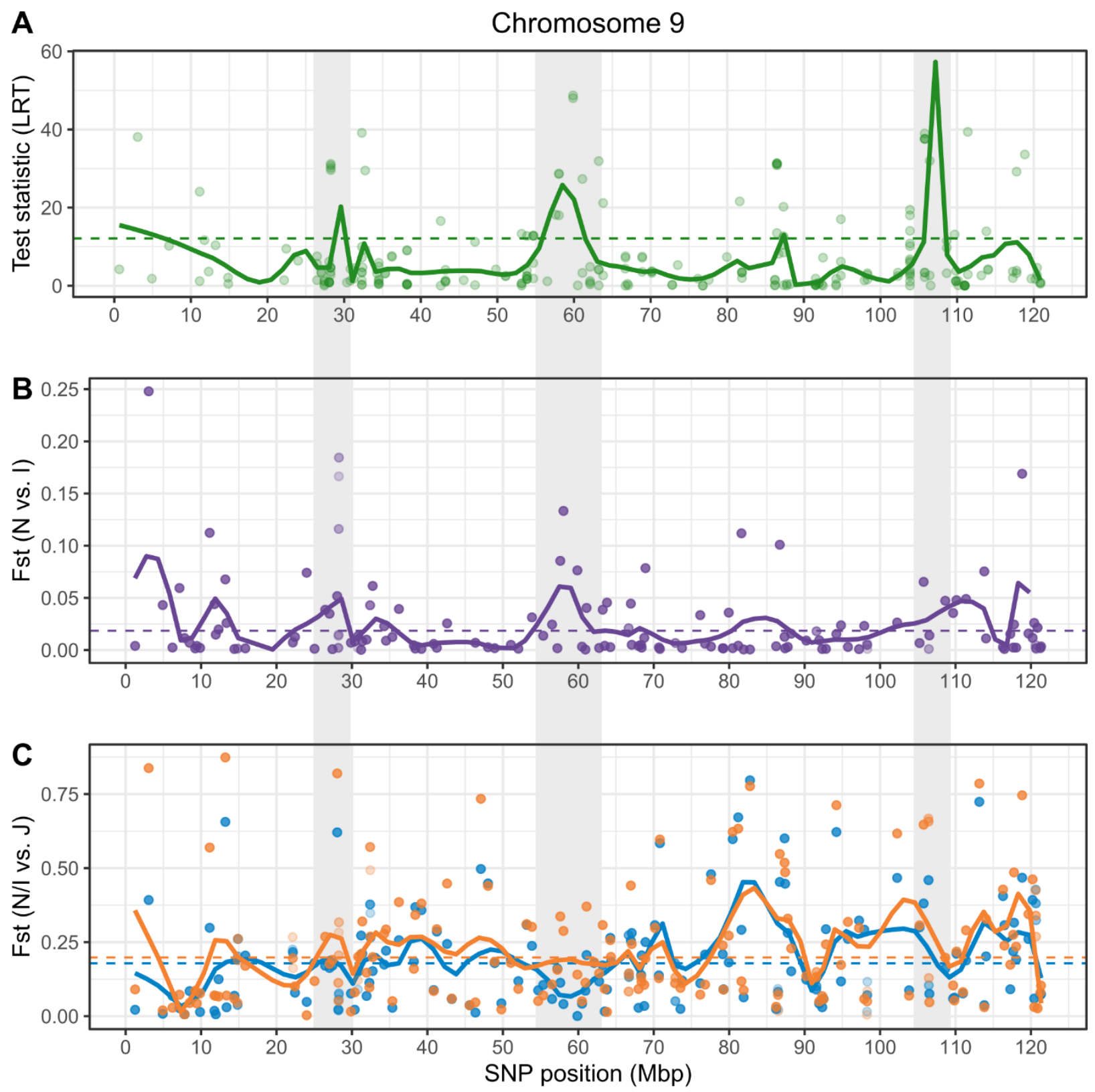

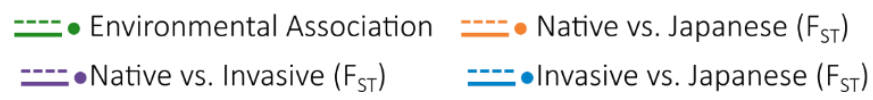

Figure 9. Genomic signatures of adaptation on chromosome 9. Genomic regions underlain with grey bars represent interesting patterns when comparing among the three panels. (A) Association of SNPs with the first environmental principle component. On the $y$-axis is the likelihood ratio test statistic, $x$-axis SNP position. The points represent single SNP association statistics; the solid line represents a locally estimated scatterplot smoothing (LOESS) curve to identify patterns in the data. The dashed line represents the significance threshold of $p=0.0005$ based on a chi-square distribution with one degree of freedom. (B) Fst between native and invasive populations. Points represent sliding window FST estimates. Again, the solid line represents a LOESS curve, while the dashed line represents the mean genome-wide Fst between the two groups. (C) Fst between native and Japanese populations in orange, between invasive and Japanese in blue. Points represent sliding window FST estimates, solid lines are LOESS curves, and dashed lines represent the mean genome-wide Fst between the given groups. 
bioRxiv preprint doi: https://doi.org/10.1101/2021.11.04.467261; this version posted November 4, 2021. The copyright holder for this preprint (which was not certified by peer review) is the author/funder, who has granted bioRxiv a license to display the preprint in perpetuity. It is made available under aCC-BY-ND 4.0 International license.
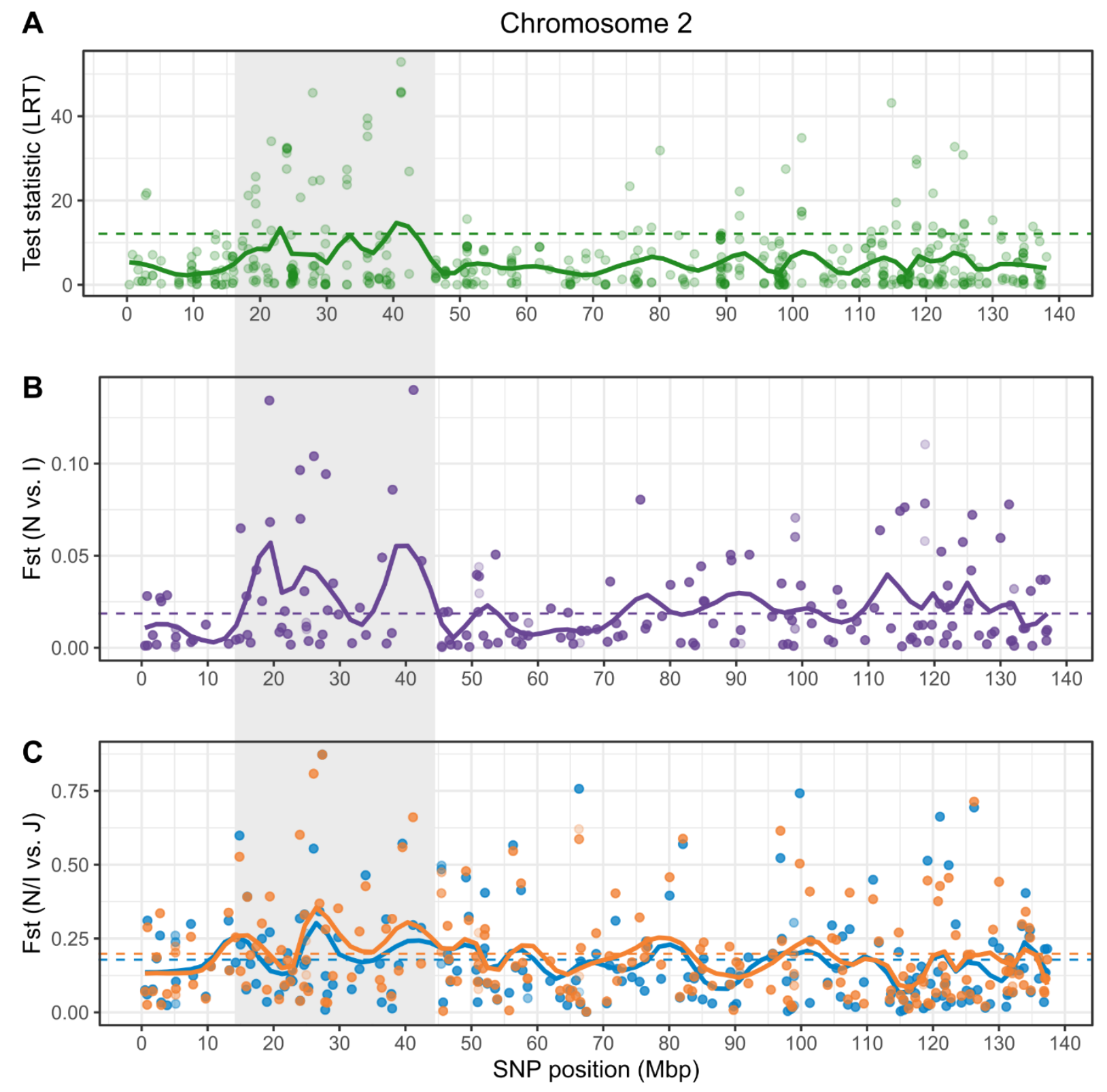

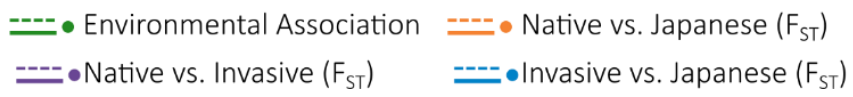

Figure 10. Genomic signatures of adaptation on chromosome 2. Genomic regions underlain with grey bars represent interesting patterns when comparing among the three panels. (A) Association of SNPs with the first environmental principle component. On the $y$-axis is the likelihood ratio test statistic, $x$-axis SNP position. The points represent single SNP association statistics; the solid line represents a LOESS curve to identify patterns in the data. The dashed line represents the significance threshold of $p=0.0005$ based on a chi-square distribution with one degree of freedom. (B) FST between native and invasive populations. Points represent sliding window FST estimates. Again, the solid line represents a LOESS curve, while the dashed line represents the mean genome-wide FST between the two groups. (C) FST between native and Japanese populations in orange, between invasive and Japanese in blue. Points represent sliding window Fst estimates, solid lines are LOESS curves, and dashed lines represent the mean genome-wide Fst between the given groups. 


\section{Discussion}

We found strong evidence that the wasp spider range expansion was facilitated by adaptive introgression, and that range edge populations are locally adapted to endure cold and highly seasonal environments at the northern range edge. We observed a latitudinal cline in adult body size and substantial genetic turnover over a very short geographic distance, suggesting very strong selection to maintain this differentiation.

\section{Core versus edge: Common garden experiment}

\section{Oviposition latency: The earlier the better}

In nearly all investigated traits, we found a significant negative effect of oviposition latency: clutches that were laid later had lower clutch sizes, lower hatching success, reduced survival, and higher SCPs. Seemingly, early oviposition is advantageous in core and edge populations. The consequence of late oviposition has been documented in A. bruennichi in the field (Leborgne \& Pasquet, 2005). That study also showed that body condition, affected by feeding, has an effect on clutch size. Thus, the negative effect of late oviposition found in our study may be a combination of an inherent decrease in clutch viability with time, as well as a lab effect due to less frequent prey acquisition in the lab. For survival and SCP, in addition to a lab effect, the effect of oviposition latency could also be explained by the time until onset of winter: with more time to prepare for winter, i.e. by accumulating cryoprotectants, offspring from earlier clutches may show greater cold tolerance and overwintering survival. In the few spider species in which seasonal variation in supercooling points has been thoroughly investigated, the accumulation of putative cryoprotectants and thermal hysteresis factors begins ahead of winter, i.e. around September in the northern hemisphere (Kirchner \& Kestler, 1969; Tanaka, 1995a). Thus, spiderlings that hatch late in September have less time to accumulate the necessary substances to suppress their supercooling points.

\section{Reproductive success}

Because egg sacs were laid and offspring hatched before we began the winter treatments, we cannot disentangle the effects of genetic adaptation and phenotypic plasticity in clutch size and hatching success, and only look at differences between origins. Females from the core of the range had larger clutches and higher hatching success than females from the edge of the range. Populations at the edge of a species range face very different selective pressures than those at the core of the range. In range-expanding species, low population densities at the expanding front, as well as predator release, are hypothesized to select for higher reproductive investment (Phillips, Brown \& Shine, 2010). In birds, clutch size generally increases with latitude, the cause of which has been the focus of many competing classical hypotheses (i.e. Lack's rule, Ashmole's hypothesis, reviewed in Lundblad \& Conway, 2021). This background led us to expect that clutch size in $A$. bruennichi would increase with latitude, which makes it all the more interesting that we find the opposite pattern. In life-history theory, clutch size is often considered in the context of a trade-off between clutch size and the level of offspring provisioning (approximated with egg size in many species) (Tanaka, 1995b; Hassall et al., 2006). However, we found that edge spiders not only have smaller clutches, they also have smaller offspring, so this tradeoff is not apparent at the full geographic scale. However, in the data overall, we did find a slight negative effect of clutch size on offspring mass: offspring from large clutches had slightly lower mass, suggesting that there is a tradeoff (Table 5). It could thus be hypothesized that edge spiders have smaller clutch sizes to optimize the peroffspring size, if resources are limited by the shorter reproductive season in northern latitudes, and that this tradeoff operates at a local scale rather than range-wide. 
Our result for clutch size contrasts with the findings of Wolz et al. (2020), where they found equal clutch size between origins in A. bruennichi; in that study, the authors suggested that because core females are larger, reproductive investment, relative to body size, is higher in edge females. In our study, we explicitly include female body size as an explanatory variable, and estimate the effects of core vs. edge at median body size. While we do find that larger females have higher clutch sizes, this is true in both populations, and core females have higher clutch sizes overall. While clutch size might naturally vary between years, one difference in our study from that of Wolz et al. is the inclusion of multiple clutches per female, and of oviposition latency as a fixed effect. If we exclude all second clutches from our data set and do not account for oviposition latency, we find the same results, that clutch size, relative to body size, is higher in edge females (Supplemental File 1: Table S2, Figure S9). Because in our study, core spiders tended to lay their egg sacs later, we may be overcorrecting for oviposition latency and inflating the estimate of core females' clutch size. However, looking at the raw data (Supplemental File 1: Figure S3A-B), our results appear to reflect the differences well: although the core females started to oviposit later, there is still substantial overlap, and their early egg sacs have higher clutch sizes than edge spiders' early egg sacs, and this is consistent over time. Oviposition latency has a very strong effect in A. bruennichi and needs to be included when investigating reproductive success in this species, to avoid drawing potentially incorrect conclusions.

\section{Genetic adaptation in cold tolerance}

We found that overwintering survival was highest under warm winter conditions for both core and edge spiderlings. However, under the cold winter treatment, edge spiderlings had higher survival than core spiderlings (Figure 2A). This suggests that the edge populations have adapted to cope with the colder winters, and plasticity does not allow the core spiderlings to cope as well under these conditions. Likewise, in our lower lethal temperature assays, edge spiderlings had higher survival at the lowest exposure temperatures. While survival was similar between origins at exposures to temperatures between -10 and $-18^{\circ} \mathrm{C}$, at more extreme cold temperatures, edge spiderlings had significantly higher survival. Across all treatments, edge spiderlings had lower LG SCPs. Slightly more nuanced is the difference in the proportion of LG:HG SCPs. At mid-winter, across both treatments and both origins, the majority of spiderlings have LG SCPs. However, post-winter, in the warm winter treatment, spiderlings from the core switch to having a majority of HG SCPs, possibly indicating a switch in strategy depending on environmental cues. This is likely adaptive for the spiderlings from the core of the range, as they can respond to environmental cues in the spring and decrease their investment in maintaining their cold tolerance when it is no longer necessary. We discuss this aspect in more detail below, in the section, "Seasonal variation in cold tolerance plasticity."

Determination of an organism's cold tolerance strategy (chilling intolerant, freeze avoidant, or freeze tolerant) requires data on the lower lethal temperature and supercooling point (Sinclair et al., 2015). Due to methodological constraints, we could not assess lethal temperature and supercooling points simultaneously in a single assay per individual. Thus, we have to compare between the two different assays to determine the $A$. bruennichi cold tolerance strategy. Pooling all of the data across treatment groups, the median SCP in A. bruennichi was $-27.93^{\circ} \mathrm{C}$. Using our model for lower lethal temperature survival, we predict $50 \%$ survival at $-27.5^{\circ} \mathrm{C}$. Thus, the median supercooling point is very similar to the average lethal temperature, suggesting that $A$. bruennichi is a freeze avoidant species: they survive until freezing, and do not succumb to the effects of cold temperatures before that point. This is consistent with 
all other reports on cold tolerance strategies of spiders from temperate habitats (Kirchner, 1973, 1987; Cubillos et al., 2018; Anthony, 2019; Anthony et al., 2019).

We also found significant differences in body size between core and edge spiderlings: edge spiderlings were smaller both in leg length and in mass. Mass varied with winter treatment, according to expectation: spiderlings had lower mass following a warm winter treatment than a cold winter treatment. Due to the linear relationship between metabolic rate and temperature in arthropods, and the fact that the spiderlings do not feed over the course of winter (Turnbull, 1973; Welke \& Schneider, 2012), they use up more of their energy reserves under warm conditions. This loss of mass continued over the course of winter, with lowest mass post-winter in the warm treatment. Edge spiderlings had consistently lower mass than core spiderlings across treatments. Lower mass in spiderlings from the edge of the range may allow for greater cold tolerance, as body size is linked to cold tolerance in many freeze avoidant arthropods: smaller individuals contain less water, and the probability of ice nucleation is directly related to the number of water molecules in the body (Lee, 2010). Thus, producing smaller offspring at the range edge may be beneficial in A. bruennichi. Another possibility is that lower mass in edge spiderlings indicates lower resource provisioning from mothers to offspring. Even if this is the case, the offspring do not seem to have lower quality (i.e. demonstrate lower survival or poorer cold tolerance capacity). Furthermore, the edge spiderlings, under their native cold winter treatment, have almost exactly the same mass as core spiderlings under their native warm winter treatment (Figure 5). Thus, edge spiderlings may have exactly the resources they need given the predictably cold winter in Estonia. However, if winter temperatures increase due to global climate change, this may prove maladaptive (Williams, Henry \& Sinclair, 2015b). We found a very strong contribution of our random effects in the model for leg length - with a conditional pseudo- $R^{2}$ of 0.817 and almost no contribution of the fixed effects (marginal pseudo- $R^{2}=0.060$ ), mother identity seems to determine offspring body size more than anything else.

Taken together, our results indicate that the edge spiderlings have evolved greater cold tolerance and smaller body size than their counterparts in the core of the range. Given that the A. bruennichi expansion began in the 1960s, and the species first arrived in Estonia in 2006 (Algo, 2010), this adaptation to the colder winter temperatures in northeastern Europe arose over the course of decades. With one generation per year, this corresponds to only a few dozen generations. Local adaptation in cold tolerance has been found in other species with large latitudinal ranges, correlated with greater variability and seasonality towards the poles (Addo-Bediako, Chown \& Gaston, 2000; Lancaster et al., 2015).

\section{Seasonal variation in cold tolerance plasticity}

In addition to the observed differences between origins, many traits showed seasonal differences, and especially seasonal differences in the degree of plasticity. For example, at mid-winter, there are no differences between the warm and cold winter treatments in lower lethal temperature. However, postwinter, survival decreases dramatically in the warm treatment at the lower exposure temperatures. This coincides closely with the SCP results. At mid-winter, there is no plasticity, i.e. no winter treatment effects, in the LG SCPs or in the proportion of LG:HG SCPs; at post-winter, there is plasticity, with higher LG SCPS in the warm treatment, and more HG SCPs in general, with a significant switch to HG SCPs in core spiderlings under the warm treatment. This is further evidence that the lower lethal temperature is related to the SCP. Furthermore, the fact that the switch toward HG SCPs was not observed in edge spiderlings suggests that adaptations have arisen to suppress plasticity in this trait and maintain a low SCP even with an early, warm spring, whereas the core spiderlings are plastic. According to theory, plasticity 
is favored under predictably variable environments, where no single phenotype has higher fitness in all environments (Ghalambor et al., 2007). Thus, plasticity loss, such as that seen in the LG:HG SCP ratio in edge spiderlings under warm conditions, should only arise when a trait is under very strong selection.

\section{Latitudinal variation in adult phenotypes and genotypes across transect Female body size, but not pigmentation, clutch size, or hatching success, varies with latitude}

We found that adult body size varies with latitudinally varying climatic variables. A. bruennichi females are smaller under colder, more seasonal environments. A. bruennichi, as many other arthropod species, follows the Converse Bergmann's rule (Mousseau, 1997; Blanckenhorn \& Demont, 2004; Chown \& Gaston, 2010; Williams, Chick \& Sinclair, 2015a). Whether this is due to a shorter growing season or a faster development time cannot be discerned in this study, but it is interesting to note that the smaller body size of edge spiders is already present at the spiderling stage.

Contrary to our expectation, given the fact that many ectothermic animals (i.e. reptiles, insects) show darker coloration at northern latitudes (Watt, 1968; Guppy, 1986; Svensson \& Waller, 2013; MartínezFreiría et al., 2020), we did not find darker opisthosoma pigmentation with increasing latitude. However, we did find a significant association of pigmentation with one environmental principal component (PC4). This PC is strongly related to the mean temperature of the wettest quarter - populations with low temperatures in the wettest quarter had less dark coloration. This effect is very difficult to interpret, and is also not particularly strong. One possible explanation for why we found no latitudinal variation, is that spiders may perform some degree of behavioral thermoregulation by orienting either their dorsal or ventral side toward the sun, as has been documented in some other spider species and within the Argiope genus (Robinson \& Robinson, 1978; Tolbert, 1979). In A. bruennichi, as in many other spider species, the ventral opisthosoma is much darker than the dorsal opisthosoma. By measuring the pigmentation of the dorsal side, we may have missed variation in ventral pigmentation, or there is simply no adaptive variation in pigmentation, as behavioral thermoregulation may be sufficient across the range. It is also possible that opisthosoma pigmentation is plastic, and may vary according to the presence or absence of sun. Some spiders are able to very rapidly alter their coloration in response to environmental stimuli, although this has never been documented in any Argiope species (reviewed by Holl, 1987). Given that we measured pigmentation from photographs taken in the field, at varying times of day and under varying environmental conditions, we are not able to test this with our data. Alternatively, although the dorsal opisthosoma pattern is a striking feature in this species, the pigmentation of the legs and/or prosoma may be more important, as these body parts contain more muscles. Darker pigmentation would allow the muscles to warm up, which may be important to optimize foraging success on sunny days, when prey insects are more active. Lastly, the dorsal opisthosoma pattern may have evolved under different, nonthermoregulatory, selection pressures, i.e. to prevent the formation of a searching image for predators, or to attract insect prey (Bond, 2007; Bush, Yu \& Herberstein, 2008).

We also did not find any pattern in hatching success or clutch size according to environmental factors. Although our results from the core and edge show that edge spiders have lower clutch sizes and hatching success, this seems not to be consistent across the whole transect. It is possible that the populations at the very edge of the range suffer from edge effects that are not reflected in intermediate populations along the transect. Another possibility is that hatching success and clutch size are highly variable within populations, and our sample size along the transect, of 10 females per population, was not high enough 
to detect a signal, given the noise. The very low pseudo- $\mathrm{R}^{2}$ values of our models for clutch size and hatching success suggest that this is likely to be the case (Table 6), and thus we prefer to interpret the core and edge results, above, where the sample sizes are much higher, as are the pseudo- $R^{2}$ values (Table 2, Table 3).

\section{Population genetic statistics show strong differentiation over a short geographic distance}

Our population clustering analyses revealed that the "native" and "invasive" populations are genetically differentiated, and this differentiation arises over a very short geographic distance. Along our transect, the turnover from "native" to "invasive" occurs over an $85 \mathrm{~km}$ stretch within the middle of Germany (Figure 7), and is far less gradual than might be expected, given the level of gene flow possible in such a dispersive species that shows overall low genetic variation across the Palearctic (Krehenwinkel et al., 2016). Our results provide strong support, in great detail, to the hypothesis presented by Krehenwinkel \& Tautz (2013): introgression occurred in populations within central Germany. We demonstrate this here by showing that there is greater genetic diversity in the "invasive" populations, and more genetic similarity of invasive populations to the Russian and Japanese populations than found in the French populations.

We calculated individual-level inbreeding coefficients, and compared these across populations. The Japanese populations were highly inbred, while Russian populations were outbred, and there were intermediate inbreeding levels within European populations (Figure 8). High inbreeding of the Japanese populations is unsurprising; $A$. bruennichi spiderlings disperse via aerial ballooning, which should be selected against in island populations, as the likelihood of dispersing into unsuitable areas, i.e. the ocean, is very high. Within the populations at the edge of the range in Estonia, we observed a linear increase in inbreeding levels with latitude. This may be explained by the lower dispersal propensity of edge populations of $A$. bruennichi found by Wolz et al. (2020). In that study, edge spiderlings were less dispersive than core spiderlings, which was in contrast to the authors' expectations of spatial sorting and Olympic village effects considered typical at the leading edge of a range expansion (Shine, Brown \& Phillips, 2011). Wolz et al. hypothesized that there may be strong selection against dispersal at the edge of the range in this case, due to possibly fragmented habitat patches and/or low population densities. Lower dispersal propensity may then lead to increased inbreeding, or vice versa. In light of our results for the lower lethal temperature and supercooling points, we find the explanation for selection against dispersal highly plausible. Further north and inland of our collecting sites, temperatures can dip below $32^{\circ} \mathrm{C}$ when arctic cold fronts move over Estonia. Such low temperatures are likely fatal for $A$. bruennichi spiderlings, even for short exposure times, based on our data. Thus, it seems that the populations in Estonia are no longer an expansion front, but have reached a range border, beyond which survival over winter is unlikely. This is consistent with the findings of many studies, that poleward range limits are set by direct environmental limitations, where adverse conditions exceed species' tolerance levels (MacArthur, 1972; Root, 1988; Parmesan et al., 2005; Mazaris et al., 2013).

\section{Support for adaptive introgression}

Throughout the genome, we found genomic windows with strong environmental associations, high differentiation of native/invasive populations in $F_{S T}$, and relatively lower $F_{S T}$ between invasive and Japanese populations. This pattern suggests that in A. bruennichi, adaptive introgression may have truly provided the genomic substrate for selection, enabling adaptation to colder winters. We found that the same regions of the genome were associated with multiple environmental principal components. In order 
to parse apart the precise environmental drivers of adaptation, we will need to test the associations with single bioclimatic variables, rather than summarized principal components. This will also allow us to see if the same genomic regions are associated with adaptation to multiple environmental factors, or if there are different patterns given different, more specific, environmental variables. Further investigation into the genes present in these regions is needed, to shed light on their potential functions. What is clear from our results is that precipitation, strongly represented in PCs 2 and 4, does not exert a strong selection pressure in this system. Temperature and seasonality, on the other hand, show more and stronger genetic associations. Although the sampling design in our study is not intended to identify the precise donor population for introgression, based on our results and those of previous studies (Krehenwinkel \& Tautz, 2013; Krehenwinkel et al., 2015), it is highly likely that the introgression came from the east, as represented here by Japanese populations, through Russia. The A. bruennichi populations in Russia are characterized by a strongly seasonal climate, with an annual temperature range of $35-40^{\circ} \mathrm{C}$, and very cold winters. Thus, it stands to reason that admixture of the European and Russian populations could have enabled introgression of alleles from eastern populations that evolved under cold and seasonal environments.

One very interesting aspect of our results is the high level of differentiation and environmental association we found on chromosome 9, which has recently been identified as an X chromosome (Sheffer et al., 2021a). A. bruennichi possesses a multiple $X$ chromosome system, where males are hemizygous for two $X$ chromosomes ( 9 and 10), meaning females have two copies of each $X$ chromosome while males have one copy of each. Therefore, the effective population size and recombination rate for $X$ chromosomes should be lower than autosomes. This leads to the theoretical prediction of increased evolutionary rates of $X$ chromosomes under natural selection, especially in the case of recessive variants (Charlesworth, Coyne \& Barton, 1987; Ellegren, 2009; Bechsgaard et al., 2019). In the case of $A$. bruennichi, it seems that at least one of the $X$ chromosomes has indeed evolved more quickly than autosomes. Chromosome 10 shows weaker patterns than chromosome 9 , and does not stand out in its range of LRT or FST values in the same way (Supplemental File 1: Figure S10). Although they have a different sex determination system, there are interesting introgression patterns in $\mathrm{X}$ chromosomes found at the house mouse (Mus musculus/M. domesticus) hybrid zone. One central region of the $X$ chromosome in the house mouse has been identified as an area of reduced gene flow, and is thought to be a candidate region for reproductive isolation, while another region shows a signature of adaptive introgression (Payseur, Krenz \& Nachman, 2004). Our work builds on the existing evidence that sex chromosomes can - and do - evolve rapidly.

\section{Conclusion}

Our study had two central aims: first, to quantify genetic adaptation and phenotypic plasticity in cold tolerance in edge relative to core populations, and second, given a strong role of genetic adaptation in enabling the establishment and persistence of expanding populations, to test if the range expansion and subsequent adaptation was enabled by adaptive introgression. Our reciprocal transplant common garden experiment revealed that the edge populations are clearly locally adapted in cold tolerance. Although there is significant plasticity, especially in the spring, edge spiderlings are more cold tolerant across all investigated traits. Our population genomic analysis revealed striking differentiation over a short geographic distance, suggesting strong selection. Going deeper, looking at genome-wide population genetic outliers and environmental associations, we find compelling, if correlative, evidence for adaptive introgression providing the substrate for selection to the colder, more seasonal environments in 
northeastern Europe. Taken together, all of our results suggest that $A$. bruennichi populations in Estonia are no longer an expanding front, but have reached a poleward range limit.

\section{Data availability}

The scripts for filtering SNPs in and outside of exons were written by KJH and can be shared upon reasonable request. The genome-wide results for all chromosomes for each environmental PC (65 graphs, thus excluded from the supplement for brevity) can be shared by MMS upon request. Observed means and variances of spider traits will be uploaded to the World Spider Trait Database (https://spidertraits.sci.muni.cz/) for utility in meta-analyses.

\section{Acknowledgements}

We would like to thank Lydia Smith and Natalie Graham at UC Berkeley for their invaluable advice on ddRAD library preparation and sequencing. We are grateful to Cynthia Wang for her work on adapting the library preparation protocol. Many thanks to Heidi Land for her incredible organizational skills helping with the coordination of animal caretaking, and more. We also thank Alexandra Machnis, Timon Möller, Birte Schadlowski, Anja Junghanns, and Julia Balk for their help with spider husbandry, counting, and measuring offspring. We also thank Marina Wolz for her very helpful advice on the overwintering procedure and how to count and measure offspring.

\section{Author contributions}

GU, HK, SP, and MMS designed the study. Samples were collected by GU, HK, LZ, BS, and MMS. DNA was extracted by HK and MMS. MMS performed the library preparation and sequencing. With help from SP and $\mathrm{KJH}, \mathrm{MMS}$ performed all population genomic analyses. LZ, BS, GU and MMS photographed the spiders in the field. Phenotyping of adult spiders was done by LZ, BS and MMS. The common garden experiment and subsequent physiological/phenotypic measurements of offspring were designed and run by MMS, with help from LZ and BS. MMS performed all statistical analysis of the adult phenotypes, environmental variables, and common garden data, with input from PM and TN.

\section{References}

Addo-Bediako, A., Chown, S.L. \& Gaston, K.J. (2000). Thermal tolerance, climatic variability and latitude. Proc. R. Soc. London. Ser. B Biol. Sci. 267, 739-745.

Ahrens, C.W., Rymer, P.D., Stow, A., Bragg, J., Dillon, S., Umbers, K.D.L. \& Dudaniec, R.Y. (2018). The search for loci under selection: trends, biases and progress. Mol. Ecol. 27, 1342-1356.

Alexander, D.H., Novembre, J. \& Lange, K. (2009). Fast model-based estimation of ancestry in unrelated individuals. Genome Res. 19, 1655-1664.

Algo, R.-J. (2010). Herilasämblik (Argiope bruennichi) ja tema sissetung Eestisse (Wasp spider Argiope bruennichi and his invasion of Estonia). Õpilaste Tead. 2, 4-26.

Anthony, S. (2019). Thermal biology of temperate and high-latitude arachnids. Electron. Thesis Diss. Repos. University of Western Ontario, London, Ontario.

Anthony, S.E., Buddle, C.M., Høye, T.T. \& Sinclair, B.J. (2019). Thermal limits of summer-collected Pardosa wolf spiders (Araneae: Lycosidae) from the Yukon Territory (Canada) and Greenland. Polar 
Biol. 42, 2055-2064.

Auld, J.R., Agrawal, A.A. \& Relyea, R.A. (2010). Re-evaluating the costs and limits of adaptive phenotypic plasticity. Proc. R. Soc. B Biol. Sci. 277, 503-511.

Barrett, R.D.H. \& Schluter, D. (2008). Adaptation from standing genetic variation. Trends Ecol. Evol. 23, 38-44.

Bates, D., Mächler, M., Bolker, B.M. \& Walker, S.C. (2015). Fitting linear mixed-effects models using Ime4. J. Stat. Softw. 67, 1-48.

Bechsgaard, J., Schou, M.F., Vanthournout, B., Hendrickx, F., Knudsen, B., Settepani, V., Schierup, M.H. \& Bilde, T. (2019). Evidence for faster X chromosome evolution in spiders. Mol. Biol. Evol. 36, 12811293.

Blanckenhorn, W.U. \& Demont, M. (2004). Bergmann and converse Bergmann latitudinal clines in arthropods: Two ends of a continuum? Integr. Comp. Biol. 44, 413-424.

Bolger, A.M., Lohse, M. \& Usadel, B. (2014). Trimmomatic: a flexible trimmer for Illumina sequence data. Bioinformatics 30, 2114-2120.

Bond, A.B. (2007). The evolution of color polymorphism: Crypticity, searching images, and apostatic selection. Annu. Rev. Ecol. Evol. Syst. 38, 489-514.

Brooks, M.E., Kristensen, K., van Benthem, K.J., Magnusson, A., Berg, C.W., Nielsen, A., Skaug, H.J., Mächler, M. \& Bolker, B.M. (2017). glmmTMB balances speed and flexibility among packages for zero-inflated generalized linear mixed modeling. $R$ J. 9, 378-400.

Bush, A.A., Yu, D.W. \& Herberstein, M.E. (2008). Function of bright coloration in the wasp spider Argiope bruennichi (Araneae: Araneidae). Proc. R. Soc. B Biol. Sci. 275, 1337-1342.

Charlesworth, B., Coyne, J.A. \& Barton, N.H. (1987). The relative rates of evolution of sex chromosomes and autosomes. Am. Nat. 130, 113-146.

Chen, I.C., Hill, J.K., Ohlemüller, R., Roy, D.B. \& Thomas, C.D. (2011). Rapid range shifts of species associated with high levels of climate warming. Science (80-. ). 333, 1024-1026.

Chown, S.L. \& Gaston, K.J. (2010). Body size variation in insects: A macroecological perspective. Biol. Rev. 85, 139-169.

Clausen, J., Keck, D.D. \& Hiesey, W.M. (1941). Regional differentiation in plant species. Am. Nat. 75, 231-250.

Colautti, R.I. \& Barrett, S.C.H. (2013). Rapid adaptation to climate facilitates range expansion of an invasive plant. Science (80-. ). 342, 364-366.

Cubillos, C., Cáceres, J.C., Villablanca, C., Villarreal, P., Baeza, M., Cabrera, R., Graether, S.P. \& Veloso, C. (2018). Cold tolerance mechanisms of two arthropods from the Andean Range of Central Chile: Agathemera crassa (Insecta: Agathemeridae) and Euathlus condorito (Arachnida: Theraphosidae). J. Therm. Biol. 74, 133-139.

Ellegren, H. (2009). The different levels of genetic diversity in sex chromosomes and autosomes. Trends Genet. 25, 278-284.

Fick, S.E. \& Hijmans, R.J. (2017). WorldClim 2: new 1-km spatial resolution climate surfaces for global 
land areas. Int. J. Climatol. 37, 4302-4315.

Follner, K. \& Klarenberg, A.J. (1995). Aeronautic behaviour in the wasp-like spider, Argiope bruennichi (Scopoli) (Araneae, Argiopidae). Proc. 15th Eur. Colloq. Arachnol. 72, 66-72.

Fordyce, J.A. (2006). The evolutionary consequences of ecological interactions mediated through phenotypic plasticity. J. Exp. Biol. 209, 2377-2383.

Fraser, D.J., Weir, L.K., Bernatchez, L., Hansen, M.M. \& Taylor, E.B. (2011). Extent and scale of local adaptation in salmonid fishes: Review and meta-analysis. Heredity (Edinb). 106, 404-420.

Fumagalli, M., Vieira, F.G., Korneliussen, T.S., Linderoth, T., Huerta-Sánchez, E., Albrechtsen, A. \& Nielsen, R. (2013). Quantifying population genetic differentiation from next-generation sequencing data. Genetics 195, 979-992.

Fumagalli, M., Vieira, F.G., Linderoth, T. \& Nielsen, R. (2014). ngsTools: Methods for population genetics analyses from next-generation sequencing data. Bioinformatics 30, 1486-1487.

Ghalambor, C.K., McKay, J.K., Carroll, S.P. \& Reznick, D.N. (2007). Adaptive versus non-adaptive phenotypic plasticity and the potential for contemporary adaptation in new environments. Funct. Ecol. 21, 394-407.

Guppy, C.S. (1986). Geographic variation in wing melanism of the butterfly Parnassius phoebus F. (Lepidoptera: Papilionidae). Can. J. Zool. 64, 956-962.

Hartig, F. (2021). DHARMa: Residual diagnostics for hierarchical (multi-level / mixed) regression models.

Hassall, M., Walters, R.J., Telfer, M. \& Hassall, M.R.J. (2006). Why does a grasshopper have fewer, larger offspring at its range limits? J. Evol. Biol. 19, 267-276.

Hedrick, P.W. (2013). Adaptive introgression in animals: examples and comparison to new mutation and standing variation as sources of adaptive variation. Mol. Ecol. 22, 4606-4618.

Hereford, J. (2009). A quantitative survey of local adaptation and fitness trade-offs. Am. Nat. 173, 579588.

Hickling, R., Roy, D.B., Hill, J.K., Fox, R. \& Thomas, C.D. (2006). The distributions of a wide range of taxonomic groups are expanding polewards. Glob. Chang. Biol. 12, 450-455.

Hoban, S., Kelley, J.L., Lotterhos, K.E., Antolin, M.F., Bradburd, G., Lowry, D.B., Poss, M.L., Reed, L.K., Storfer, A. \& Whitlock, M.C. (2016). Finding the genomic basis of local adaptation: Pitfalls, practical solutions, and future directions. Am. Nat. 188, 379-397.

Hoffmann, A.A. \& Bridle, J. (2021). The dangers of irreversibility in an age of increased uncertainty: revisiting plasticity in invertebrates. Oikos.

Holl, A. (1987). Coloration and chromes. In Ecophysiology of Spiders: 16-25. Nentwig, W. (Ed). . Berlin Heidelberg New York Tokyo: Springer-Verlag.

Huerta-Sánchez, E., Jin, X., Asan, Bianba, Z., Peter, B.M., Vinckenbosch, N., Liang, Y., Yi, X., He, M., Somel, M., Ni, P., Wang, B., Ou, X., Huasang, Luosang, J., Cuo, Z.X.P., Li, K., Gao, G., Yin, Y., Wang, W., Zhang, X., Xu, X., Yang, H., Li, Y., Wang, J., Wang, J. \& Nielsen, R. (2014). Altitude adaptation in Tibetans caused by introgression of Denisovan-like DNA. Nature 512, 194-197.

Johnson, P.L.F. \& Slatkin, M. (2008). Accounting for bias from sequencing error in population genetic 
estimates. Mol. Biol. Evol. 25, 199-206.

Kassambara, A. \& Mundt, F. (2020). FactoExtra: Extract and visualize the results of multivariate data analyses.

Kirchner, W. (1973). Ecological aspects of cold resistance in spiders (a comparative study). In Effects of temperature on ectothermic organisms: 271-279. Wieser, W. (Ed). . Berlin, Heidelberg: Springer.

Kirchner, W. (1987). Behavioural and Physiological Adaptations to Cold. In Ecophysiology of Spiders: 6677. Nentwig, W. (Ed). . Berlin, Heidelberg: Springer.

Kirchner, W. \& Kestler, P. (1969). Untersuchungen zur Kälteresistenz der Schilfradspinne Araneus cornutus (Araneidae). J. Insect Physiol. 15, 41-53.

Kopelman, N.M., Mayzel, J., Jakobsson, M., Rosenberg, N.A. \& Mayrose, I. (2015). CLUMPAK: A program for identifying clustering modes and packaging population structure inferences across K. Mol. Ecol. Resour. 15, 1179-1191.

Korneliussen, T.S., Albrechtsen, A. \& Nielsen, R. (2014). ANGSD: Analysis of Next Generation Sequencing Data. BMC Bioinformatics 15, 356.

Korneliussen, T.S. \& Moltke, I. (2015). NgsRelate: a software tool for estimating pairwise relatedness from next-generation sequencing data. Bioinformatics 31, 4009-4011.

Krehenwinkel, H., Graze, M., Rödder, D., Tanaka, K., Baba, Y.G., Muster, C. \& Uhl, G. (2016). A phylogeographical survey of a highly dispersive spider reveals eastern Asia as a major glacial refugium for Palaearctic fauna. J. Biogeogr. 43, 1583-1594.

Krehenwinkel, H., Rödder, D. \& Tautz, D. (2015). Eco-genomic analysis of the poleward range expansion of the wasp spider Argiope bruennichi shows rapid adaptation and genomic admixture. Glob. Chang. Biol. 21, 4320-4332.

Krehenwinkel, H. \& Tautz, D. (2013). Northern range expansion of European populations of the wasp spider Argiope bruennichi is associated with global warming-correlated genetic admixture and population-specific temperature adaptations. Mol. Ecol. 22, 2232-2248.

Lancaster, L.T., Dudaniec, R.Y., Chauhan, P., Wellenreuther, M., Svensson, E.I. \& Hansson, B. (2016). Gene expression under thermal stress varies across a geographical range expansion front. Mol. Ecol. 25, 1141-1156.

Lancaster, L.T., Dudaniec, R.Y., Hansson, B. \& Svensson, E.I. (2015). Latitudinal shift in thermal niche breadth results from thermal release during a climate-mediated range expansion. J. Biogeogr. 42, 1953-1963.

Leborgne, R. \& Pasquet, A. (2005). Time of oviposition and reproductive success in Argiope bruennichi (Araneae: Araneidae). Eur. J. Entomol. 102, 169-174.

Lee, R.E. (2010). A primer on insect cold-tolerance. In Low Temperature Biology of Insects: 3-34. Denlinger, D.L. \& Lee, R.E. (Eds). . Cambridge University Press.

Lefort, V., Desper, R. \& Gascuel, O. (2015). FastME 2.0: A comprehensive, accurate, and fast distancebased phylogeny inference program. Mol. Biol. Evol. 32, 2798-2800.

Leimu, R. \& Fischer, M. (2008). A meta-analysis of local adaptation in plants. PLoS One 3, e4010. 
Lepais, O. \& Weir, J.T. (2014). SimRAD: An R package for simulation-based prediction of the number of loci expected in RADseq and similar genotyping by sequencing approaches. Mol. Ecol. Resour. 14, 1314-1321.

Li, H. (2013). Aligning sequence reads, clone sequences and assembly contigs with BWA-MEM. arXiv.

Li, H., Handsaker, B., Wysoker, A., Fennell, T., Ruan, J., Homer, N., Marth, G., Abecasis, G. \& Durbin, R. (2009). The Sequence Alignment/Map format and SAMtools. Bioinformatics 25, 2078-2079.

Lüdecke, D. (2018). ggeffects: Tidy data frames of marginal effects from regression models. J. Open Source Softw. 3, 772.

Lüdecke, D., Ben-Shachar, M., Patil, I., Waggoner, P. \& Makowski, D. (2021). performance: An R package for assessment, comparison and testing of statistical models. J. Open Source Softw. 6, 3139.

Lundblad, C.G. \& Conway, C.J. (2021). Ashmole's hypothesis and the latitudinal gradient in clutch size. Biol. Rev. 96, 1349-1366.

Lynch, M. (2008). Estimation of nucleotide diversity, disequilibrium coefficients, and mutation rates from high-coverage genome-sequencing projects. Mol. Biol. Evol. 25, 2409-2419.

Maas, D.L., Prost, S., Bi, K., Smith, L.L., Armstrong, E.E., Aji, L.P., Toha, A.H.A., Gillespie, R.G. \& Becking, L.E. (2018). Rapid divergence of mussel populations despite incomplete barriers to dispersal. Mol. Ecol. 27, 1556-1571.

MacArthur, R.H. (1972). Geographical Ecology: Patterns in the Distribution of Species. Princeton: Princeton University Press.

Martínez-Freiría, F., Toyama, K.S., Freitas, I. \& Kaliontzopoulou, A. (2020). Thermal melanism explains macroevolutionary variation of dorsal pigmentation in Eurasian vipers. Sci. Rep. 10, 1-10.

Mazaris, A.D., Kallimanis, A.S., Pantis, J.D. \& Hays, G.C. (2013). Phenological response of sea turtles to environmental variation across a species' northern range. Proc. R. Soc. B Biol. Sci. 280.

Meisner, J. \& Albrechtsen, A. (2018). Inferring population structure and admixture proportions in lowdepth NGS data. Genetics 210, 719-731.

Moest, M., Van Belleghem, S.M., James, J.E., Salazar, C., Martin, S.H., Barker, S.L., Moreira, G.R.P., Merot, C., Joron, M., Nadeau, N.J., Steiner, F.M. \& Jiggins, C.D. (2020). Selective sweeps on novel and introgressed variation shape mimicry loci in a butterfly adaptive radiation. PLoS Biol. 18.

Mousseau, T.A. (1997). Ectotherms Follow the Converse to Bergmann's Rule. Evolution (N. Y). 51, 630.

Muhlfeld, C.C., Kalinowski, S.T., McMahon, T.E., Taper, M.L., Painter, S., Leary, R.F. \& Allendorf, F.W. (2009). Hybridization rapidly reduces fitness of a native trout in the wild. Biol. Lett. 5, 328-331.

Nakagawa, S. \& Schielzeth, H. (2013). A general and simple method for obtaining R2 from generalized linear mixed-effects models. Methods Ecol. Evol. 4, 133-142.

Norris, L.C., Main, B.J., Lee, Y., Collier, T.C., Fofana, A., Cornel, A.J. \& Lanzaro, G.C. (2015). Adaptive introgression in an African malaria mosquito coincident with the increased usage of insecticidetreated bed nets. Proc. Natl. Acad. Sci. U. S. A. 112, 815-820.

Paradis, E. \& Schliep, K. (2019). ape 5.0: An environment for modern phylogenetics and evolutionary analyses in R. Bioinformatics 35, 526-528. 
Parmesan, C., Gaines, S., Gonzalez, L., Kaufman, D.M., Kingsolver, J., Peterson, A.T. \& Sagarin, R. (2005). Empirical perspectives on species borders: from traditional biogeography to global change. Oikos $108,58-75$.

Parmesan, C., Ryrholm, N., Stefanescu, C., Hill, J.K., Thomas, C.D., Descimon, H., Huntley, B., Kaila, L., Kullberg, J., Tammaru, T., Tennent, W.J., Thomas, J.A. \& Warren, M. (1999). Poleward shifts in geographical ranges of butterfly species associated with regional warming. Nature 399, 579-583.

Payseur, B.A., Krenz, J.G. \& Nachman, M.W. (2004). Differential patterns of introgression across the $X$ chromosome in a hybrid zone between two species of house mice. Evolution (N. Y). 58, 2064-2078.

Peterson, B.K., Weber, J.N., Kay, E.H., Fisher, H.S. \& Hoekstra, H.E. (2012). Double digest RADseq: An inexpensive method for de novo SNP discovery and genotyping in model and non-model species. PLoS One 7.

Phillips, B.L., Brown, G.P. \& Shine, R. (2010). Life-history evolution in range-shifting populations. Ecology 91, 1617-1627.

Pritchard, J.K., Stephens, M. \& Donnelly, P. (2000). Inference of Population Structure Using Multilocus Genotype Data. Genetics 155, 945-959.

R Core Team. (2017). R: A language and environment for statistical computing.

Racimo, F., Sankararaman, S., Nielsen, R. \& Huerta-Sánchez, E. (2015). Evidence for archaic adaptive introgression in humans. Nat. Rev. Genet. 16, 359-371.

Rambaut, A. \& Drummond, A. (2018). FigTree: Tree figure drawing tool.

Robinson, M.H. \& Robinson, B.C. (1978). Thermoregulation in orb-web spiders: New descriptions of thermoregulatory postures and experiments on the effects of posture and coloration. Zool. J. Linn. Soc. 64, 87-102.

Root, T. (1988). Environmental factors associated with avian distributional boundaries. J. Biogeogr. 15, 489-505.

Schielzeth, H. (2010). Simple means to improve the interpretability of regression coefficients. Methods Ecol. Evol. 1, 103-113.

Schneider, J.M., Fromhage, L. \& Uhl, G. (2005). Extremely short copulations do not affect hatching success in Argiope bruennichi (Araneae, Araneidae). J. Arachnol. 33, 663-669.

Sexton, J.P., McIntyre, P.J., Angert, A.L. \& Rice, K.J. (2009). Evolution and ecology of species range limits. Annu. Rev. Ecol. Evol. Syst. 40, 415-436.

Sheffer, M.M., Cordellier, M., Forman, M., Grewoldt, M., Hoffmann, K., Jensen, C., Kotz, M., Král, J., Kuss, A.W., Líznarová, E. \& Uhl, G. (2021a). Identification of sex chromosomes using genomic and cytogenetic methods in a range-expanding spider, Argiope bruennichi (Araneae: Araneidae). bioRxiv 1-26.

Sheffer, M.M., Hoppe, A., Krehenwinkel, H., Uhl, G., Kuss, A.W., Jensen, L., Jensen, C., Gillespie, R.G., Hoff, K.J. \& Prost, S. (2021b). Chromosome-level reference genome of the European wasp spider Argiope bruennichi: A resource for studies on range expansion and evolutionary adaptation. Gigascience 10, 1-12. 
Shine, R., Brown, G.P. \& Phillips, B.L. (2011). An evolutionary process that assembles phenotypes through space rather than through time. Proc. Natl. Acad. Sci. 108, 5708-5711.

Sinclair, B.J., Coello Alvarado, L.E. \& Ferguson, L. V. (2015). An invitation to measure insect cold tolerance: Methods, approaches, and workflow. J. Therm. Biol. 53, 180-197.

Sinclair, B.J., Klok, C.J., Scott, M.B., Terblanche, J.S. \& Chown, S.L. (2003). Diurnal variation in supercooling points of three species of Collembola from Cape Hallett, Antarctica. J. Insect Physiol. 49, 1049-1061.

Skotte, L., Korneliussen, T.S. \& Albrechtsen, A. (2012). Association testing for next-generation sequencing data using score statistics. Genet. Epidemiol. 36, 430-437.

Skotte, L., Korneliussen, T.S. \& Albrechtsen, A. (2013). Estimating individual admixture proportions from next generation sequencing data. Genetics 195, 693-702.

Svensson, E.I. \& Waller, J.T. (2013). Ecology and sexual selection: Evolution of wing pigmentation in calopterygid damselflies in relation to latitude, sexual dimorphism, and speciation. Am. Nat. 182.

Tanaka, K. (1995a). Seasonal change in glycogen and inositol/sorbitol contents of the house spider, Achaearanea tepidariorum (Araneae: Theridiidae). Comp. Biochem. Physiol. -- Part B Biochem. 110, 539-545.

Tanaka, K. (1995b). Variation in offspring size within a population of the web-building spider Agelena limbata. Res. Popul. Ecol. (Kyoto). 37, 197-202.

Tolbert, W.W.. (1979). Thermal stress of the orb-weaving spider Argiope trifasciata (Araneae). Oikos 32, 386-392.

Turnbull, A.L. (1973). Ecology of the true spiders (Araneomorphae). Annu. Rev. Entomol. 18, 305-348.

Uhl, G. \& Vollrath, F. (2000). Extreme body size variability in the golden silk spider (Nephila edulis) does not extend to genitalia. J. Zool. 251, 7-14.

Vieira, F.G., Fumagalli, M., Albrechtsen, A. \& Nielsen, R. (2013). Estimating inbreeding coefficients from NGS data: Impact on genotype calling and allele frequency estimation. Genome Res. 23, 18521861.

Vieira, F.G., Lassalle, F., Korneliussen, T.S. \& Fumagalli, M. (2016). Improving the estimation of genetic distances from Next-Generation Sequencing data. Biol. J. Linn. Soc. 117, 139-149.

Watt, W.B. (1968). Adaptive signficance of pigment polymorphisms in Colias butterflies. I. Variation of melanin pigment in relation to thermoregulation. Int. J. Org. Evol. 22, 437-458.

Wawer, W., Rutkowski, R., Krehenwinkel, H., Lutyk, D., Pusz-Boscheńska, K. \& Bogdanowicz, W. (2017). Population structure of the expansive wasp spider (Argiope bruennichi) at the edge of its range. J. Arachnol. 45, 361-369.

Welke, K.W. \& Schneider, J.M. (2010). Males of the orb-web spider Argiope bruennichi sacrifice themselves to unrelated females. Biol. Lett. 6, 585-588.

Welke, K.W. \& Schneider, J.M. (2012). Sexual cannibalism benefits offspring survival. Anim. Behav. 83, 201-207.

Whitman, D. \& Agrawal, A.A. (2009). What is Phenotypic Plasticity and Why is it Important? In 
Phenotypic plasticity of insects: Mechanisms and consequences: 1-63. Whitman, D. \& Ananthakrishnan, T.N. (Eds). . Enfield: Science Publishers, Inc.

Williams, C.M., Chick, W.D. \& Sinclair, B.J. (2015a). A cross-seasonal perspective on local adaptation: Metabolic plasticity mediates responses to winter in a thermal-generalist moth. Funct. Ecol. 29, 549-561.

Williams, C.M., Henry, H.A.L. \& Sinclair, B.J. (2015b). Cold truths: How winter drives responses of terrestrial organisms to climate change. Biol. Rev. 90, 214-235.

Wolz, M., Klockmann, M., Schmitz, T., Pekár, S., Bonte, D. \& Uhl, G. (2020). Dispersal and life-history traits in a spider with rapid range expansion. Mov. Ecol. 8, 1-11.

Zhang, W., Dasmahapatra, K.K., Mallet, J., Moreira, G.R.P. \& Kronforst, M.R. (2016). Genome-wide introgression among distantly related Heliconius butterfly species. Genome Biol. 17, 25. 


\section{Supplemental File 1}

Southern France Climate Simulation

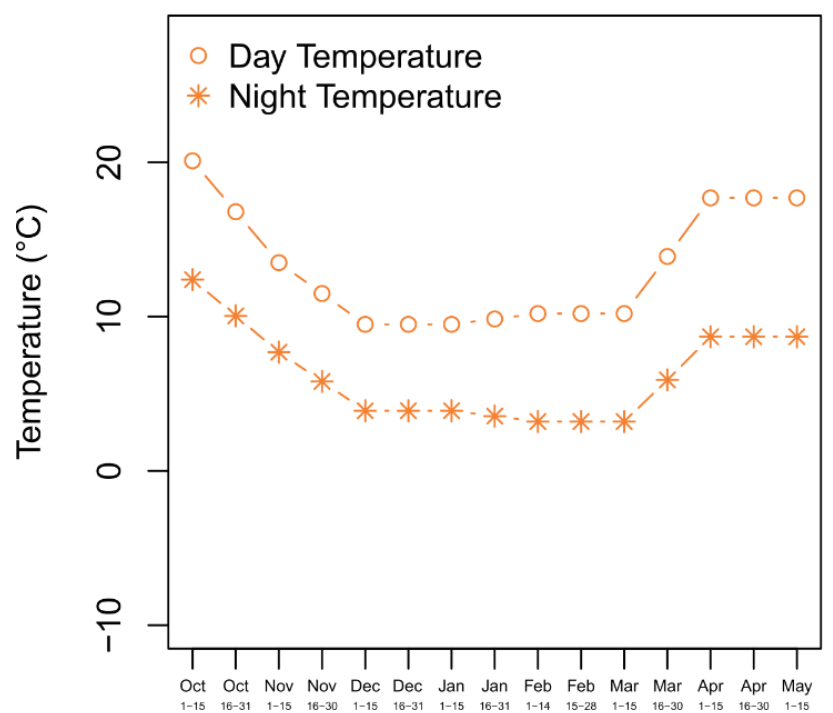

Estonia Climate Simulation

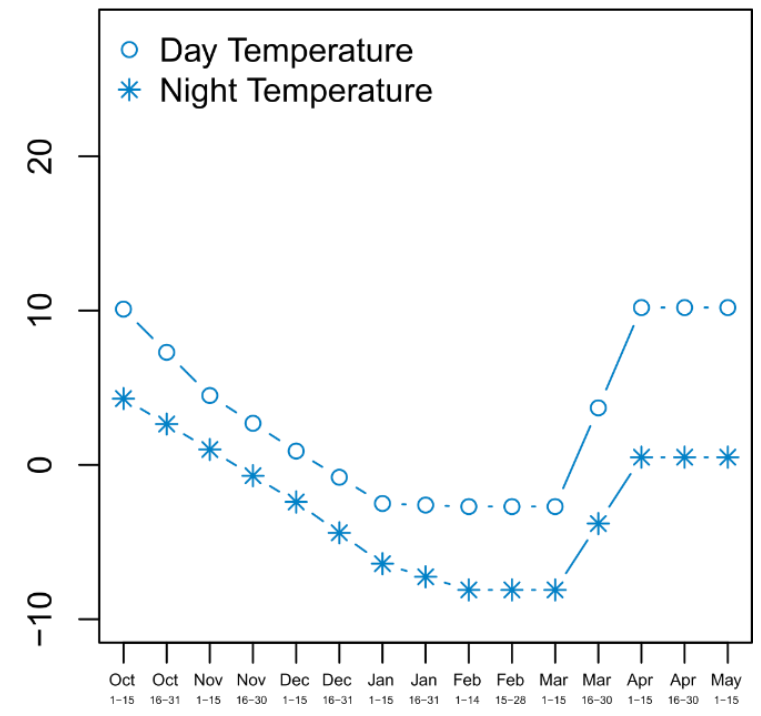

Figure S1. Climate simulations used for overwintering in our reciprocal common garden transplant. Spiderlings were placed under either conditions found at the core of the range (Southern France) or at the edge of the range (Estonia). Simulations are based on data averaged over 10 years (2004-2014), derived from www.weatheronline.co.uk

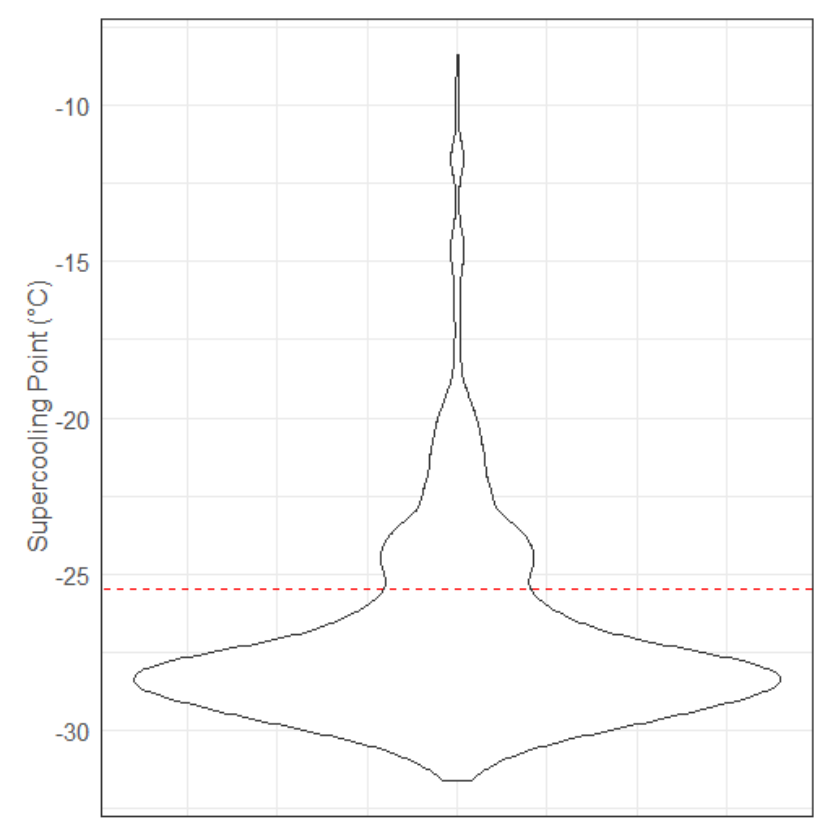

Figure S2. Multimodal distribution of supercooling points (SCPS). We split the data into two groups: low group (LG) SCPs, below $-25.5^{\circ} \mathrm{C}$ (dashed red line), and high group (HG) SCPs, above $-25.5^{\circ} \mathrm{C}$. The LG distribution was Gaussian, and we only used the HG SCPS to calculate a per-family ratio of LG:HG SCPS. 
bioRxiv preprint doi: https://doi.org/10.1101/2021.11.04.467261; this version posted November 4, 2021. The copyright holder for this preprint (which was not certified by peer review) is the author/funder, who has granted bioRxiv a license to display the preprint in perpetuity. It is made available under aCC-BY-ND 4.0 International license.
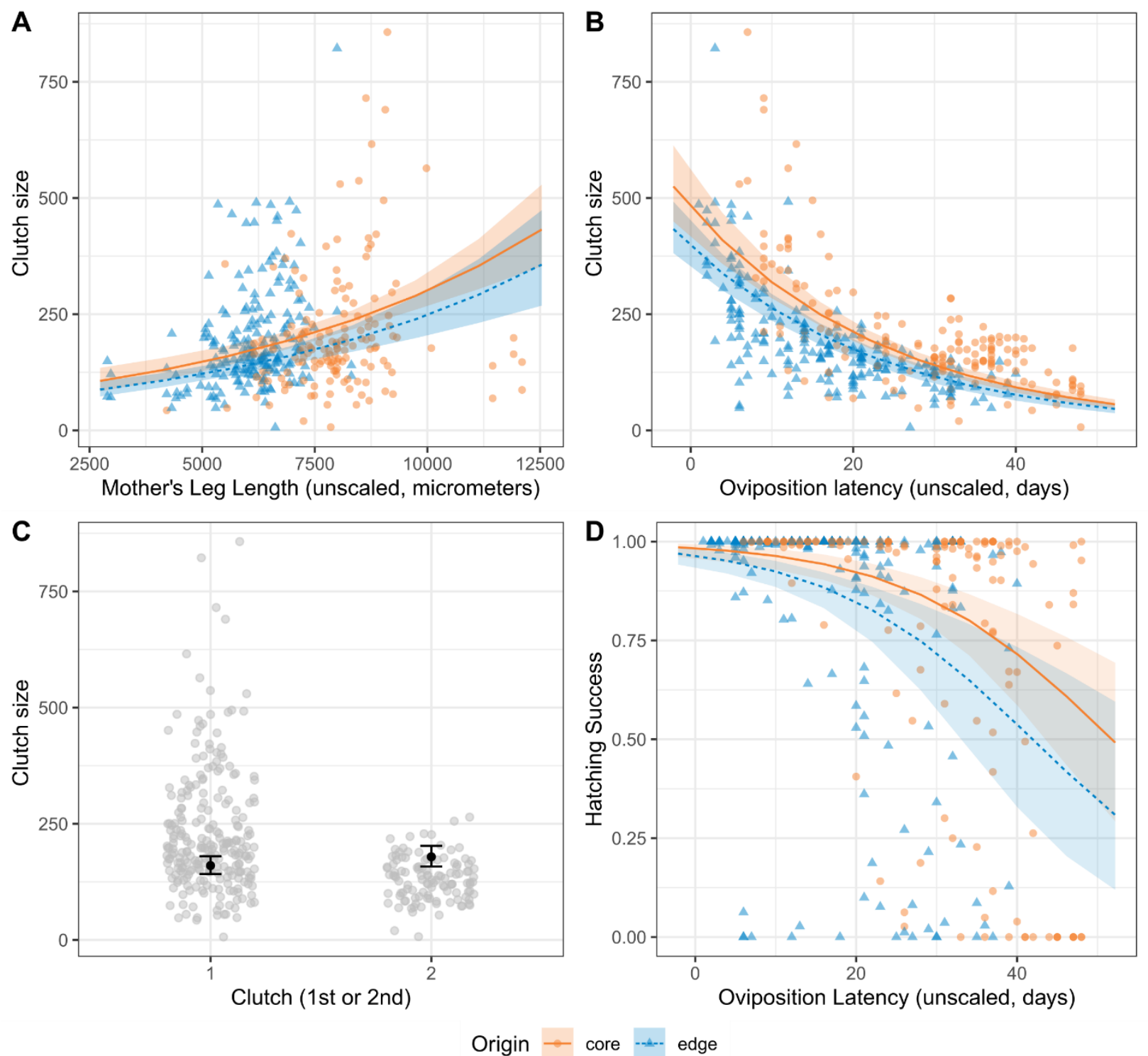

Figure S3. Clutch size and hatching success depending on mother's leg length, oviposition latency, and clutch. Transparent points represent the raw data; lines represent model predictions flanked with their $95 \%$ confidence intervals. Black points with error bars represent estimated marginal means and $95 \%$ confidence intervals for discrete predictor variables. Model predictions are back-transformed to the observed scale. 
bioRxiv preprint doi: https://doi.org/10.1101/2021.11.04.467261; this version posted November 4, 2021. The copyright holder for this preprint (which was not certified by peer review) is the author/funder, who has granted bioRxiv a license to display the preprint in perpetuity. It is made available under aCC-BY-ND 4.0 International license.

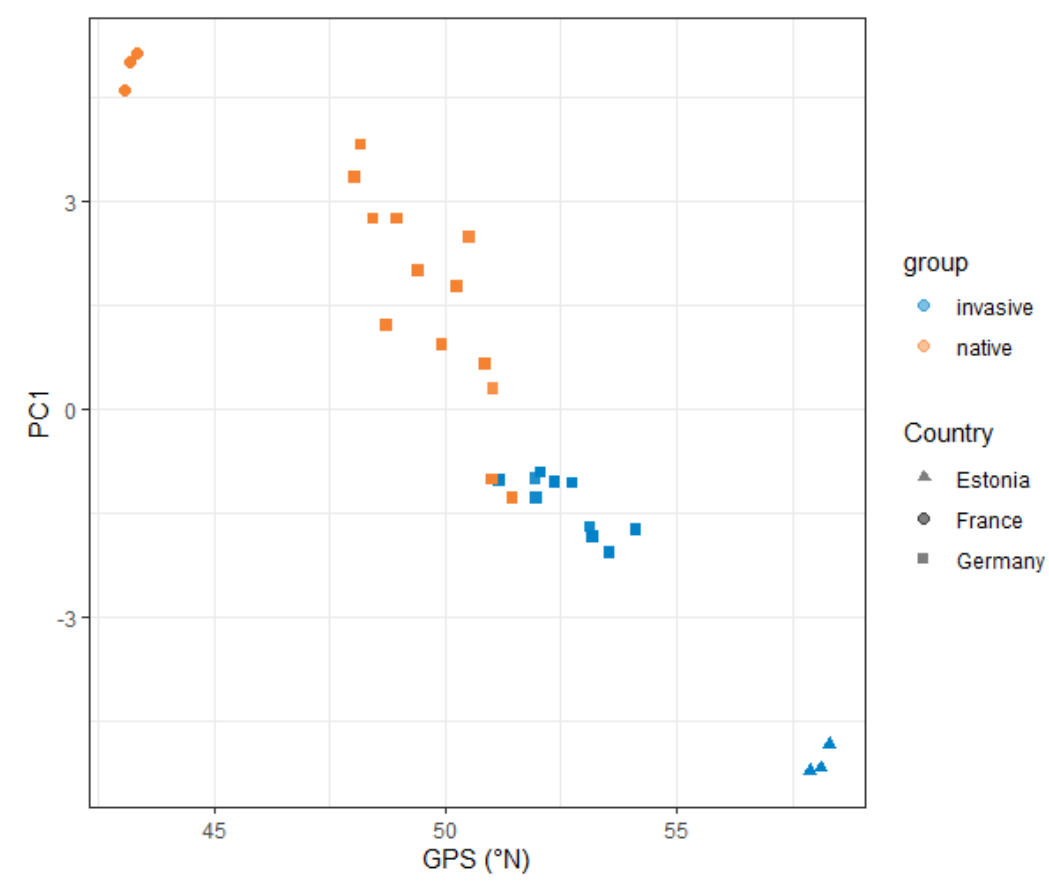

Figure S4. Environmental PC1 varies with latitude. Points represent single collecting sites, and are colored according to their assignment to "native" (orange) or "invasive" (blue) genetic clusters. Shapes represent countries.

A PC1 and PC2, with Japan

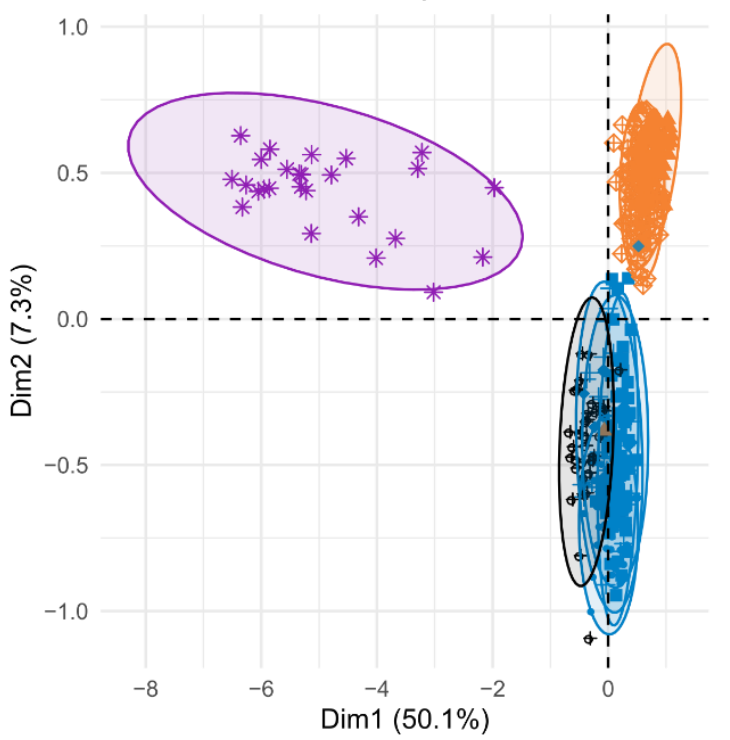

\begin{tabular}{llll}
\hline$\Delta$ & France & + & NE Germany \\
\cline { 1 - 1 } & SW Germany & Poland
\end{tabular}
B PC1 and PC3, with Japan

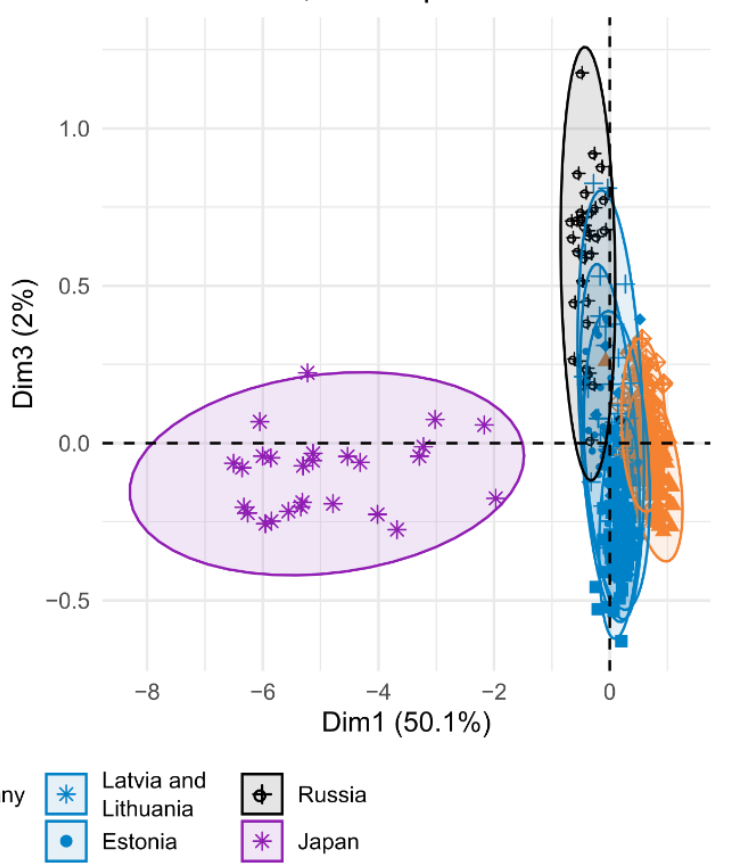

Figure S5. Principle components analysis of all populations using neutral SNPs. Ellipses represent $95 \%$ confidence intervals; points represent individual samples, with shape indicating the sampling country. Points and ellipses are colored according to the site's grouping region, Japan (purple), Russia (black), "invasive" (blue), "native" (orange). 
bioRxiv preprint doi: https://doi.org/10.1101/2021.11.04.467261; this version posted November 4, 2021. The copyright holder for this preprint (which was not certified by peer review) is the author/funder, who has granted bioRxiv a license to display the preprint in perpetuity. It is made available under aCC-BY-ND 4.0 International license.

A PC1 and PC2, without Japan

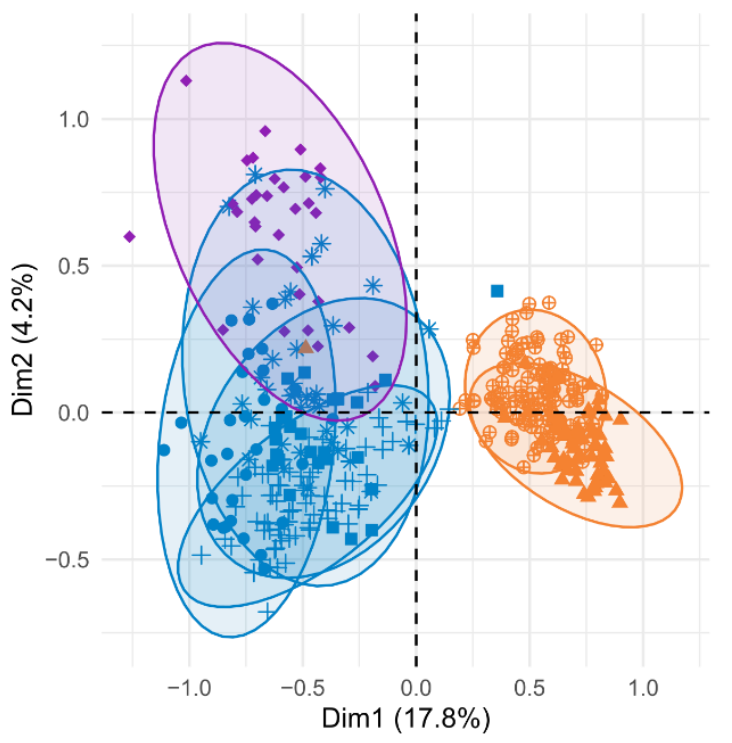

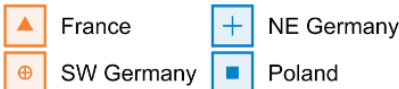

B $\quad$ PC1 and PC3, without Japan

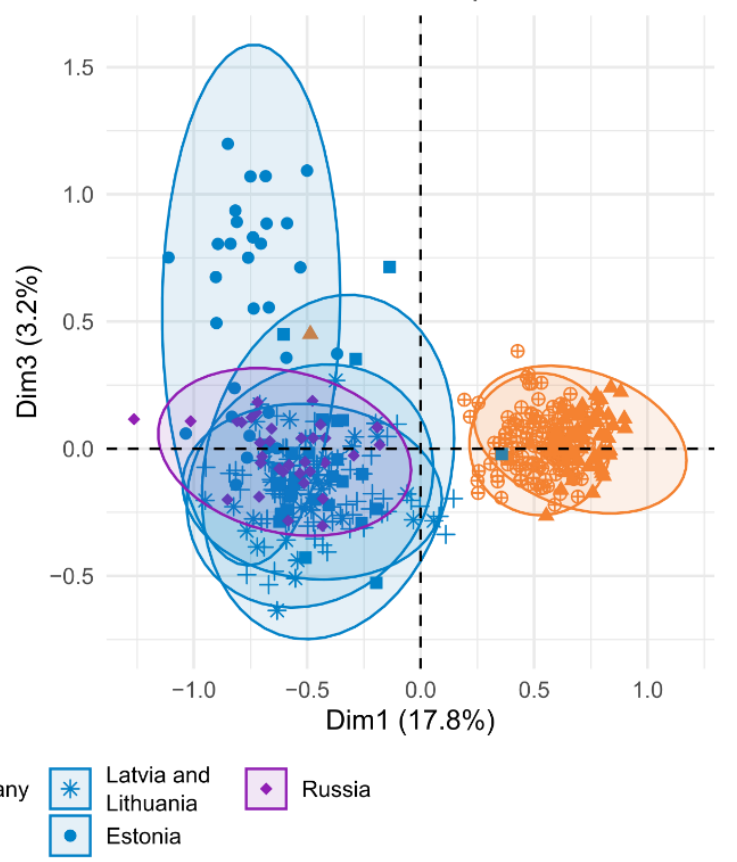

Figure S6. Principle components analysis of all non-Japanese populations using neutral SNPs. Ellipses represent 95\% confidence intervals; points represent individual samples, with shape indicating the sampling country. Points and ellipses are colored according to the site's grouping region, Russia (purple), "invasive" (blue), "native" (orange). 
bioRxiv preprint doi: https://doi.org/10.1101/2021.11.04.467261; this version posted November 4, 2021. The copyright holder for this preprint (which was not certified by peer review) is the author/funder, who has granted bioRxiv a license to display the preprint in perpetuity. It is made available under aCC-BY-ND 4.0 International license.
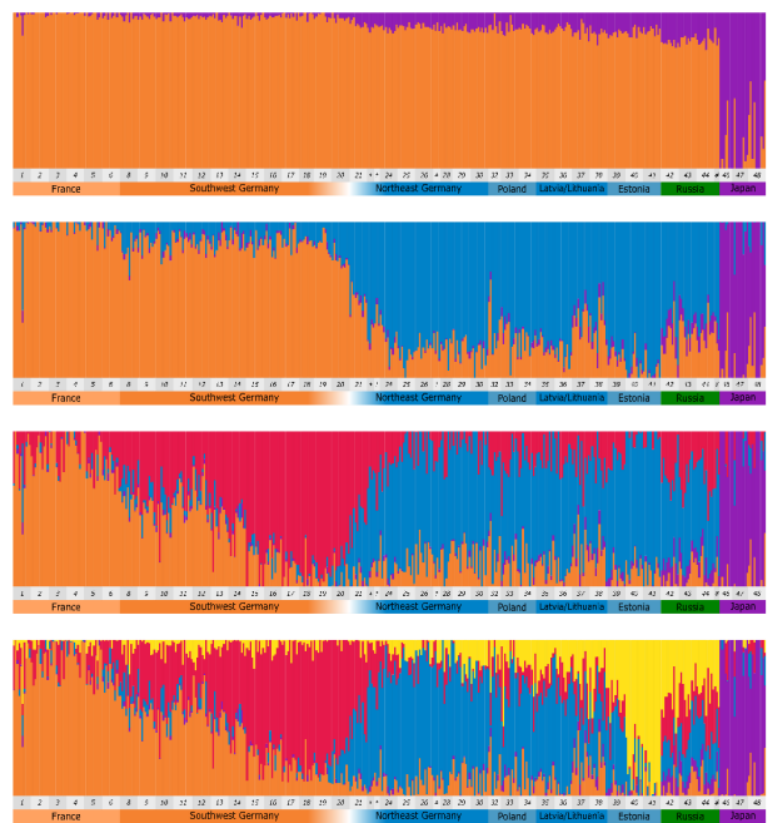
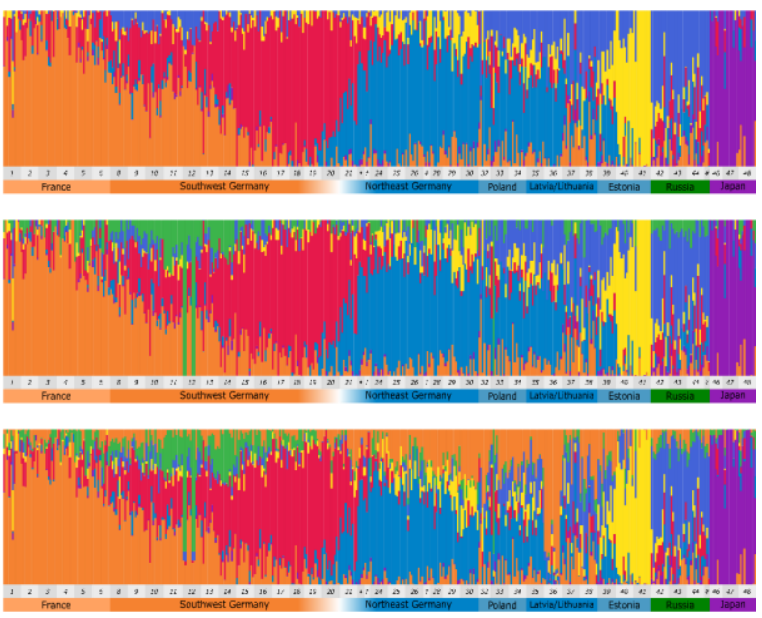

Figure S7. Stacked barplots of individual ancestry assignment, using ngsAdmix results for K=2 through $\mathrm{K}=8$.

Table S1. Pairwise FST matrix for all populations. Cells are colored by value (low: yellow, high: red). Populations are numbered as in Table 1 in the main text.

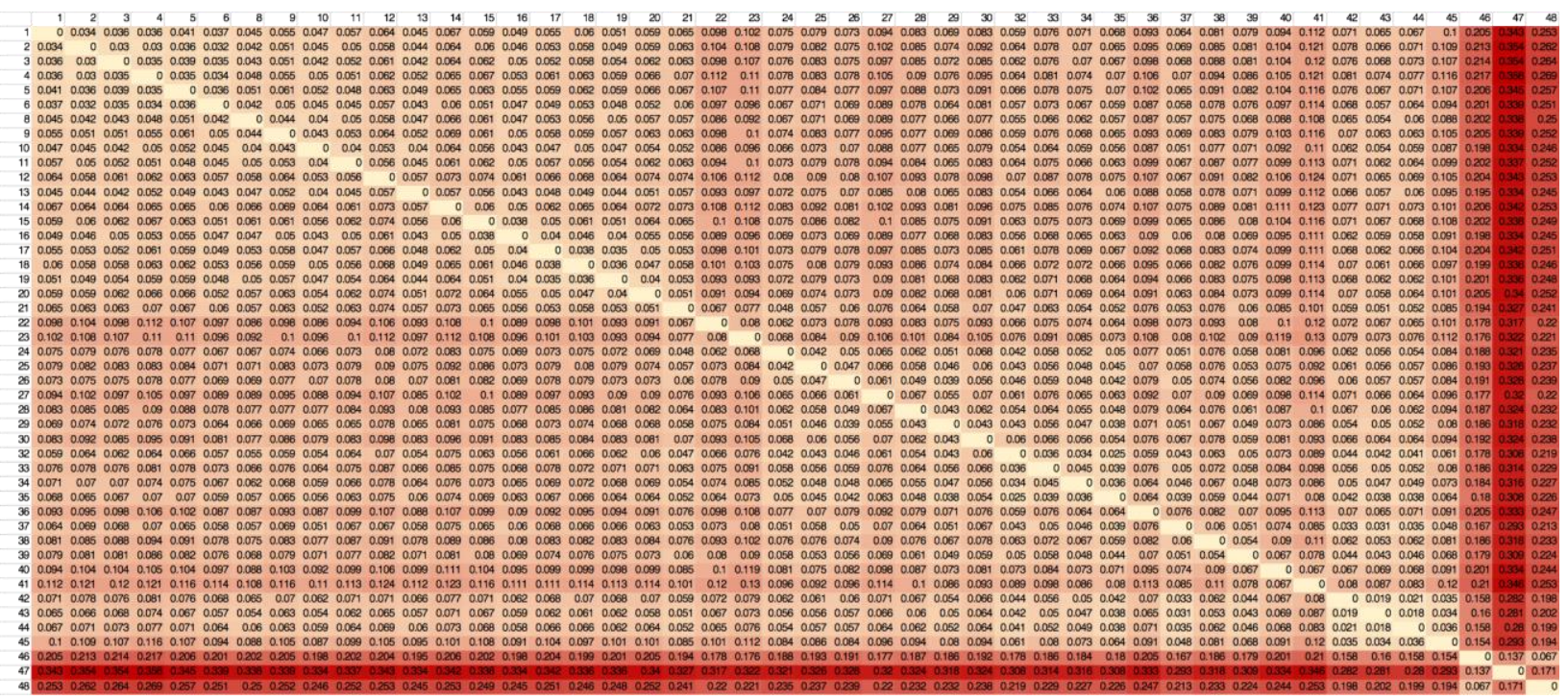


bioRxiv preprint doi: https://doi org/10 1101/2021 11 04 467261: this version posted November 4 2021. The copvriaht holder for this preprint (which was not certified by peer review) is the author/funder, who has granted bioRxiv a license to display the preprint in perpetuity. It is made available under aCC-BY-ND 4.0 International license.

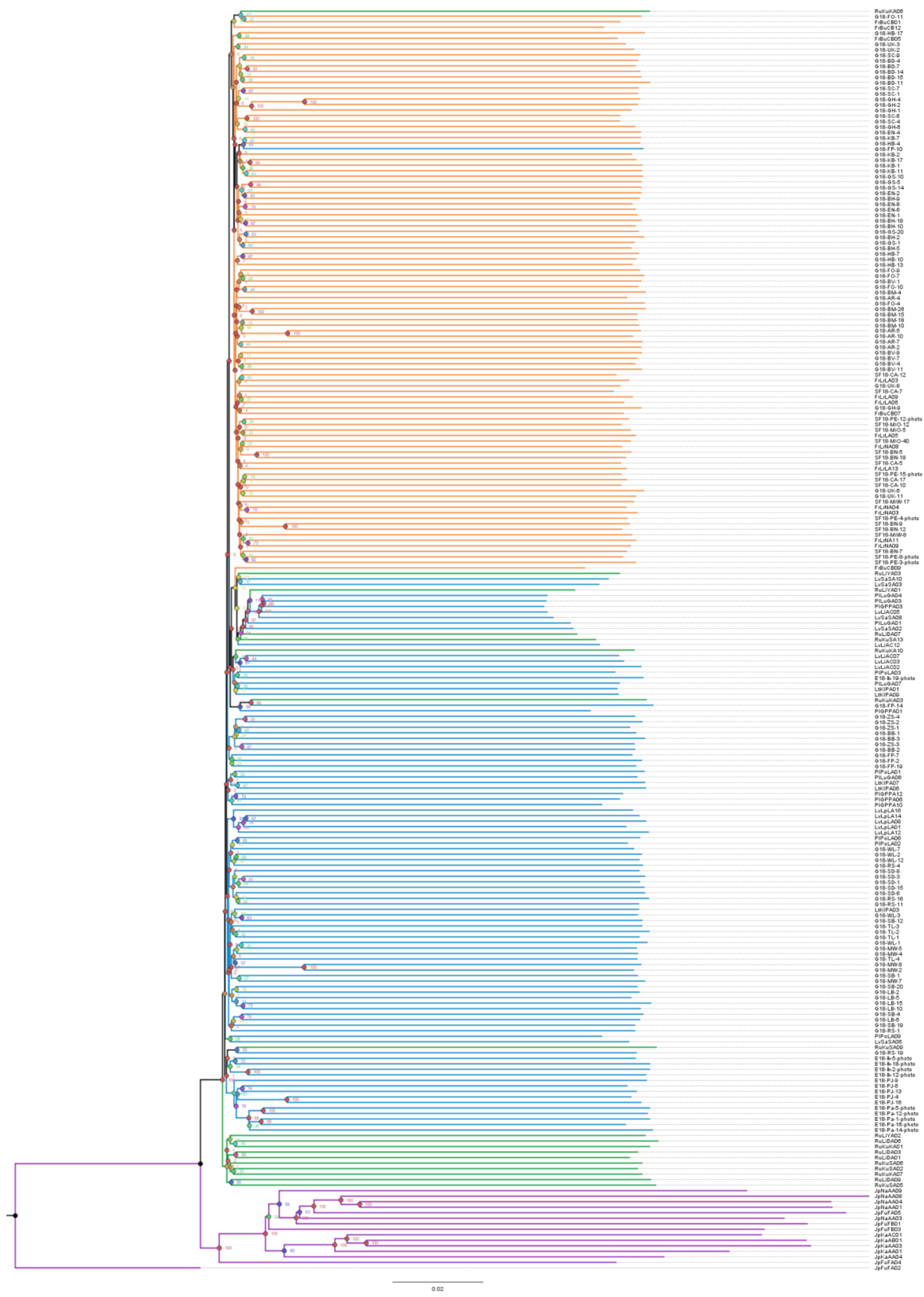

Figure S8. Phylogenetic tree based on ngsDist and FastME. Node labels are colored by bootstrap support. Branches are colored by the individual's assignment to regional groupings: Japan (purple), Russia (green), "invasive" (blue), "native" (orange). 
bioRxiv preprint doi: https://doi.org/10.1101/2021.11.04.467261; this version posted November 4, 2021. The copyright holder for this preprint (which was not certified by peer review) is the author/funder, who has granted bioRxiv a license to display the preprint in perpetuity. It is made available under aCC-BY-ND 4.0 International license.

Table S2. Model table for clutch size, if we do not include $2^{\text {nd }}$ clutches or oviposition latency. Estimates are reported on the latent scale, with $95 \%$ confidence intervals. Fixed effects are considered to be significant if their confidence interval does not cross zero; these are indicated in bold text. Reference levels for fixed effects are given in [square brackets].

\begin{tabular}{llllllll}
\hline $\begin{array}{l}\text { Response } \\
\text { Variable }\end{array}$ & $\begin{array}{l}\text { Sample } \\
\text { size }\end{array}$ & $\begin{array}{l}\text { Pseudo- } R^{2} \\
\text { (cond.) }\end{array}$ & $\begin{array}{l}\text { Pseudo-R } \\
\text { (marg.) }\end{array}$ & Fixed effects & Estimate & $2.5 \%$ & $97.5 \%$ \\
\hline $\begin{array}{l}\text { Clutch } \\
\text { size }\end{array}$ & 244 & 0.230 & 0.185 & Origin [edge] & 0.493875 & 0.21490 & 0.772849 \\
\cline { 3 - 7 } & & & & Mother's leg length* & 0.314927 & 0.21427 & 0.415588 \\
\end{tabular}

*centered and scaled

\section{A Predicted counts of Total}

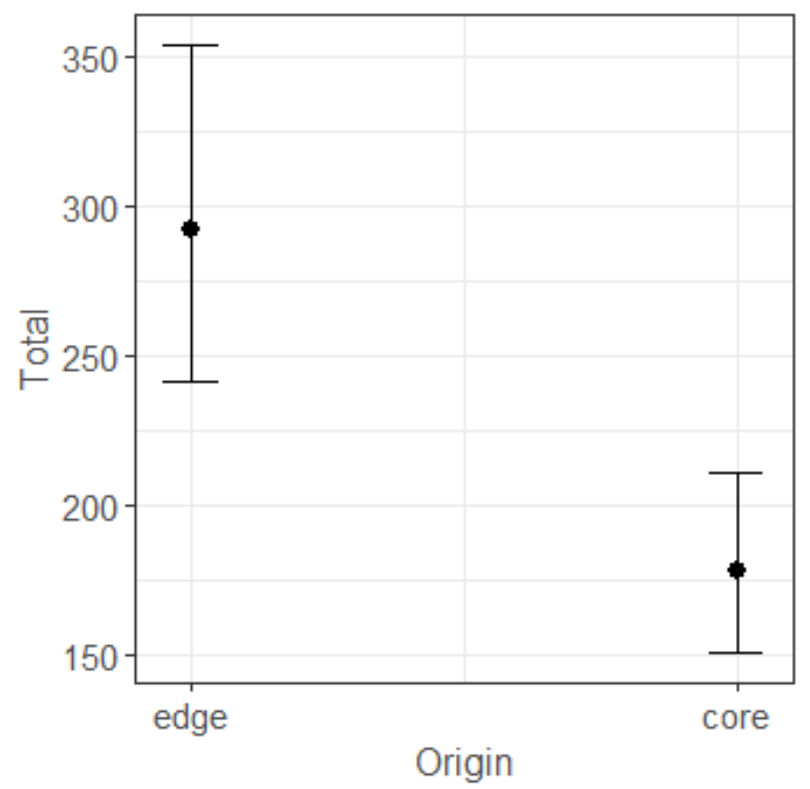

\section{B Predicted counts of Total}

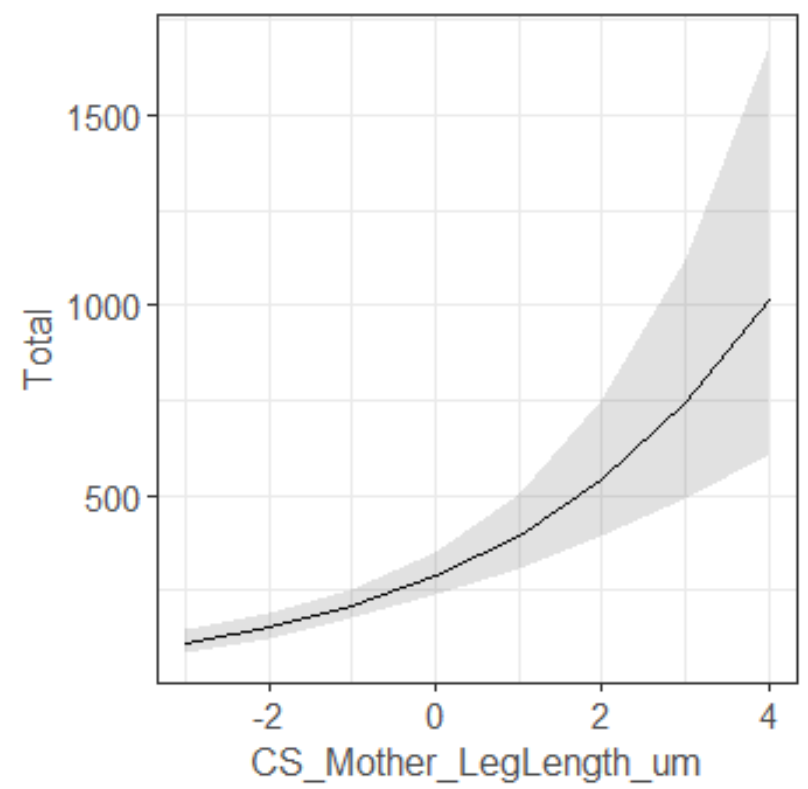

Figure 59. Model predictions for total clutch size ("Total," i.e. total number of eggs), in our supplementary exploratory model. We excluded $2^{\text {nd }}$ clutches as well as the fixed effect "Oviposition latency" and only included origin (A) and mother's body size (B) as explanatory variables. The x-axis in (B) - "CS_Mother_LegLength_um" - represents centered and scaled leg length. Black points with error bars represent estimated marginal means and $95 \%$ confidence intervals for discrete predictor variables. Model predictions are back-transformed to the observed scale. 


\section{Association with PC1}

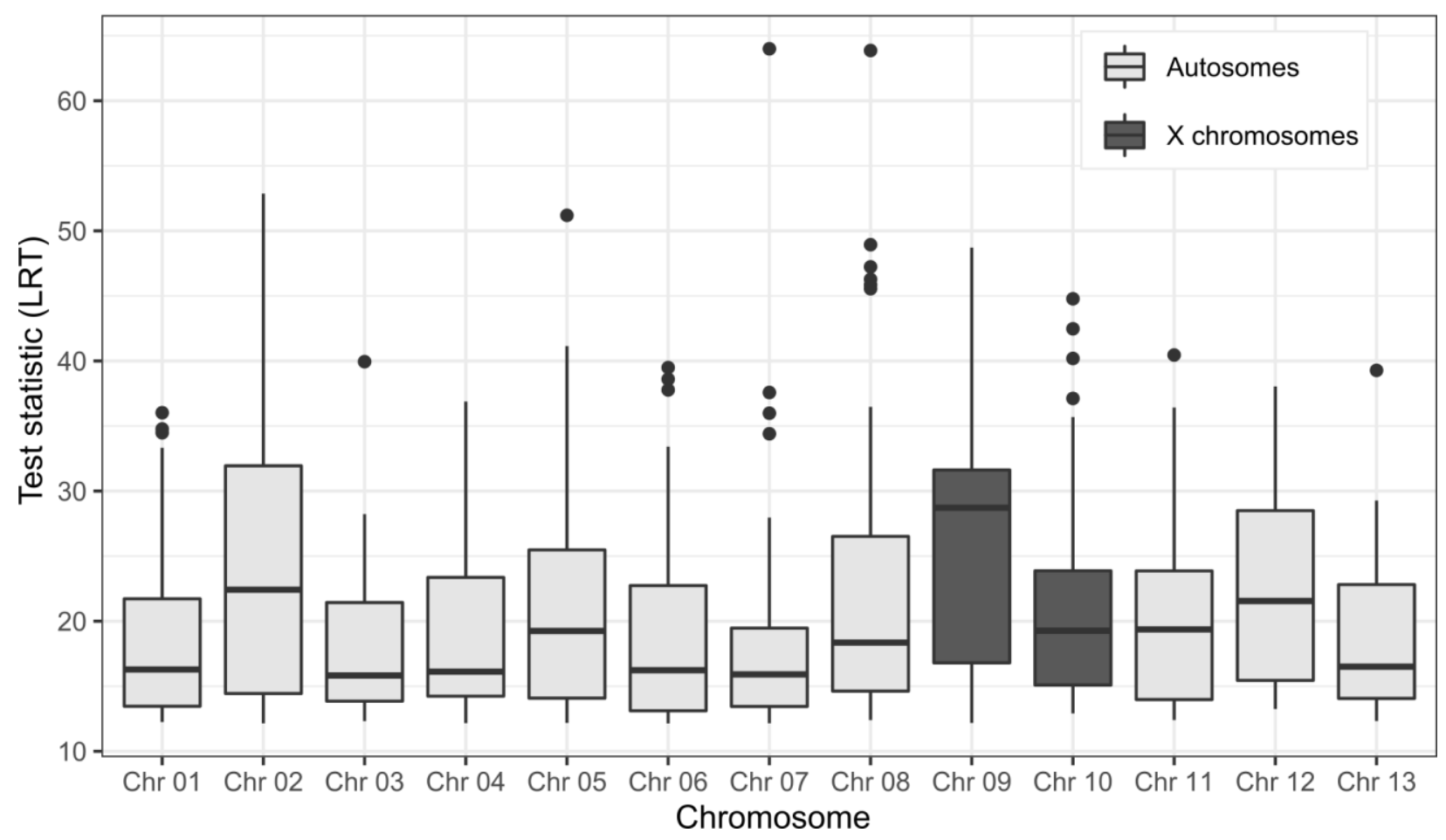

Figure S10. Boxplots of significant associations of SNPs with environmental PC1. Chromosome 9, one of the X chromosomes, shows more and stronger associations with the environment than autosomes. For the purpose of visualization, we excluded non-significant SNPs from this plot. 


\section{Supplemental File 2}

\section{Script to get Supercooling Point}

By: Brian Schulze \& Monica M. Sheffer

\section{Obtain supercooling points from datalogger data}

First, load libraries

library(openxlsx)

library(grDevices)

Then, set the threshold temperature change in degrees $C$ to detect the heat of crystallization and read in the data file (edit file path to where your data is stored, or use file.choose())

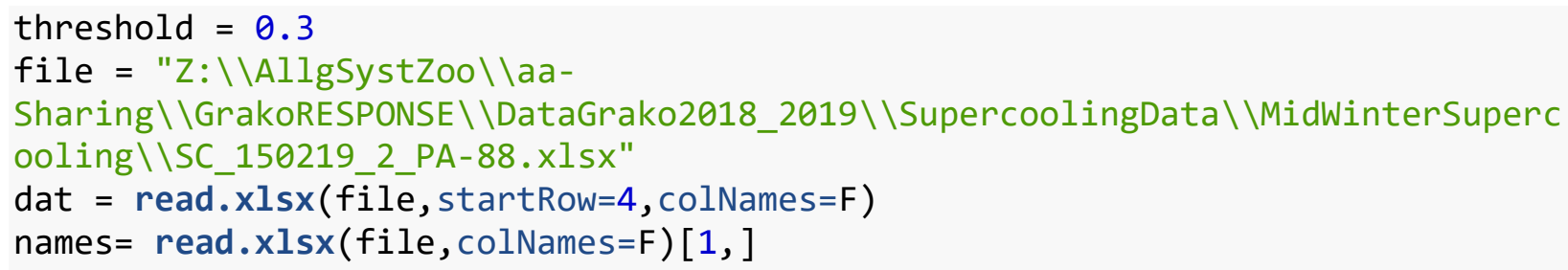

Now plot the data to get an overview, and then click on the plot to set new limits to zoom in on the peaks

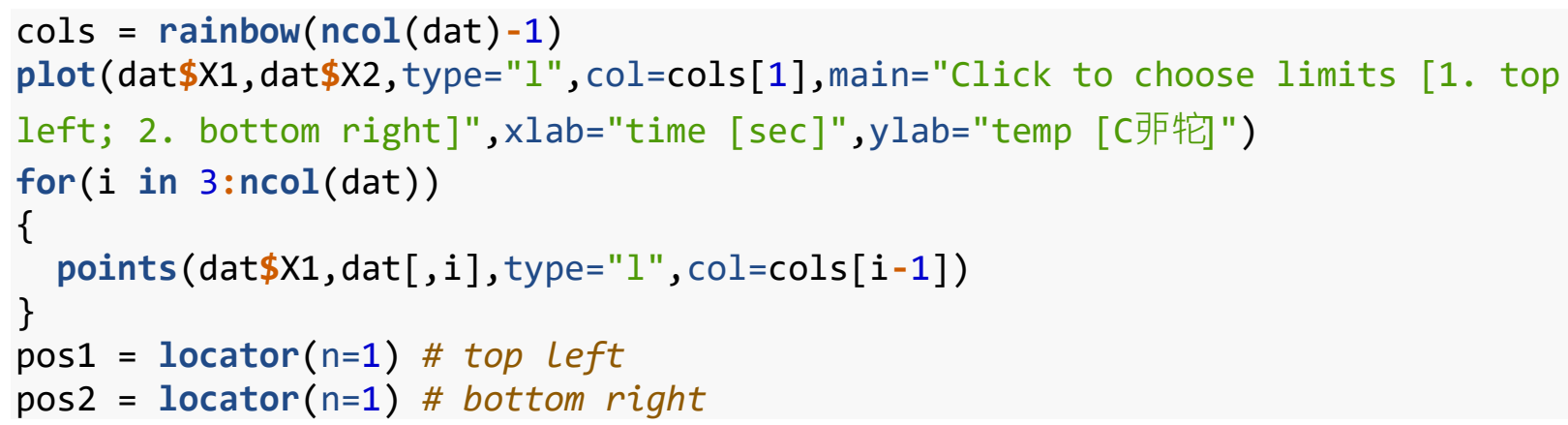




\section{Click to choose limits [1. top left; 2. bottom right]}

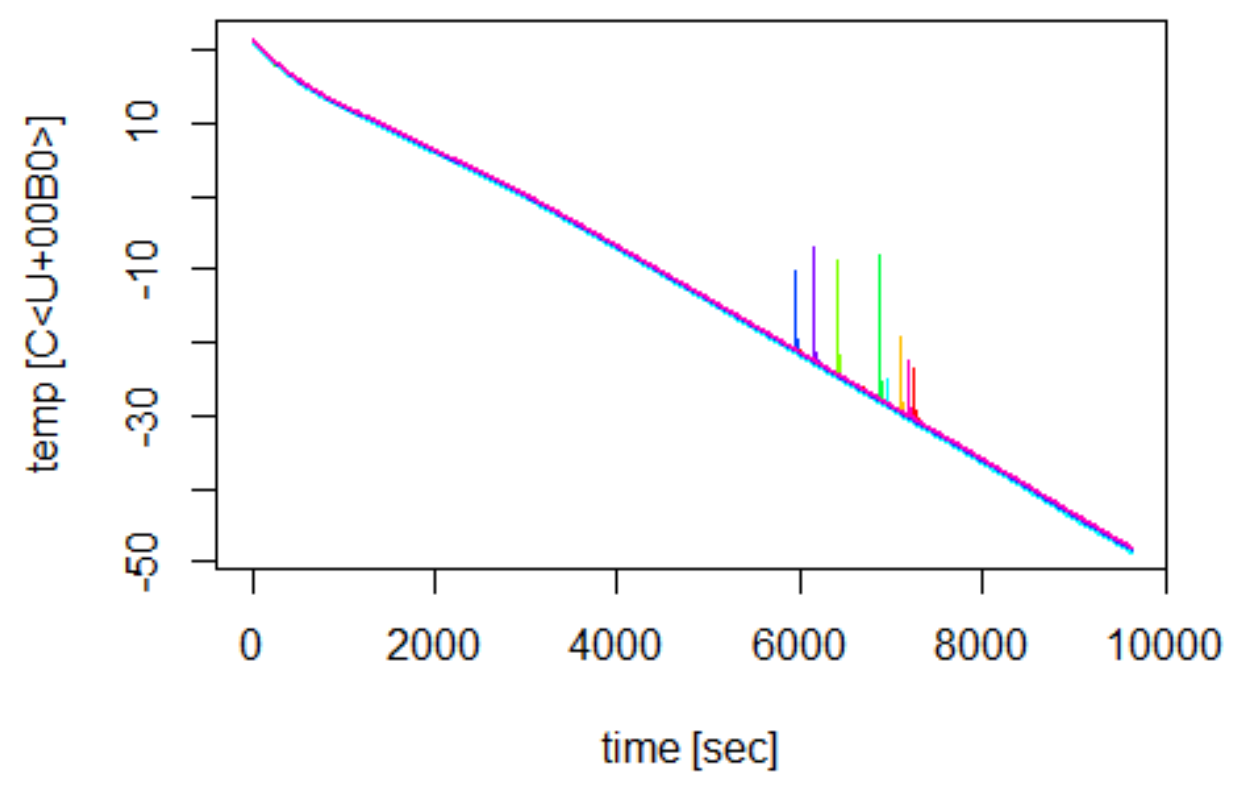

Plot the peaks with new limits obtained above, for a closer visual inspection of the data

\#normally we click on the plots, but interactive interfaces don't work with Rmarkdown, so we have to specify the positions manually in this example pos1 $=$ list $(x=4298.419, y=-9.202457)$ pos2=list $(x=7626.119, y=-33.03759)$

plot $(\operatorname{dat} \$ X 1, \operatorname{dat} \$ X 2$, type $=" 1 ", \operatorname{col}=\operatorname{cols}[1], x \lim =c(\operatorname{round}(\operatorname{pos} 1 \$ x), \operatorname{round}(\operatorname{pos} 2 \$ x)), y$

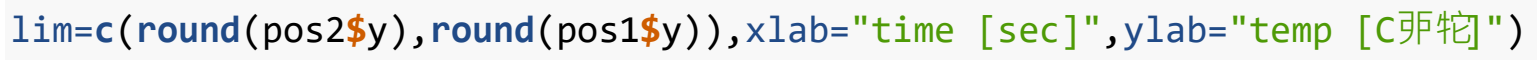
for ( $i$ in $3: n \operatorname{col}(d a t)$ )

\{ \}

points (dat\$X1, dat $[, i]$, type="1", col=cols $[i-1])$

\#Having checked the data visually (everything looks good), now calculate the SCPS, as well as the height of the peak and area below the curve, using the threshold set above

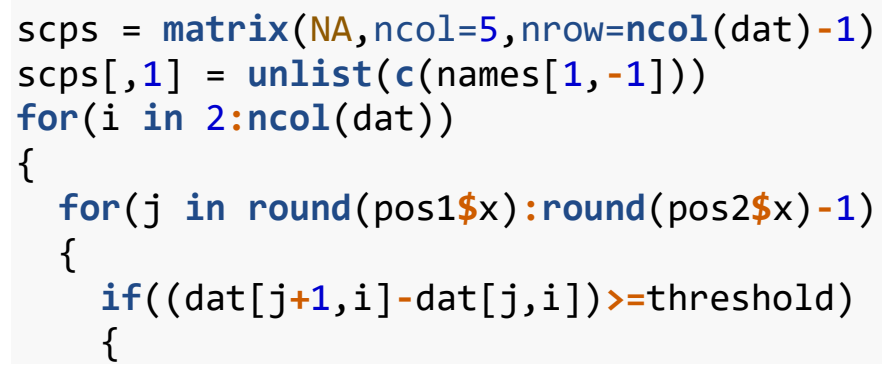




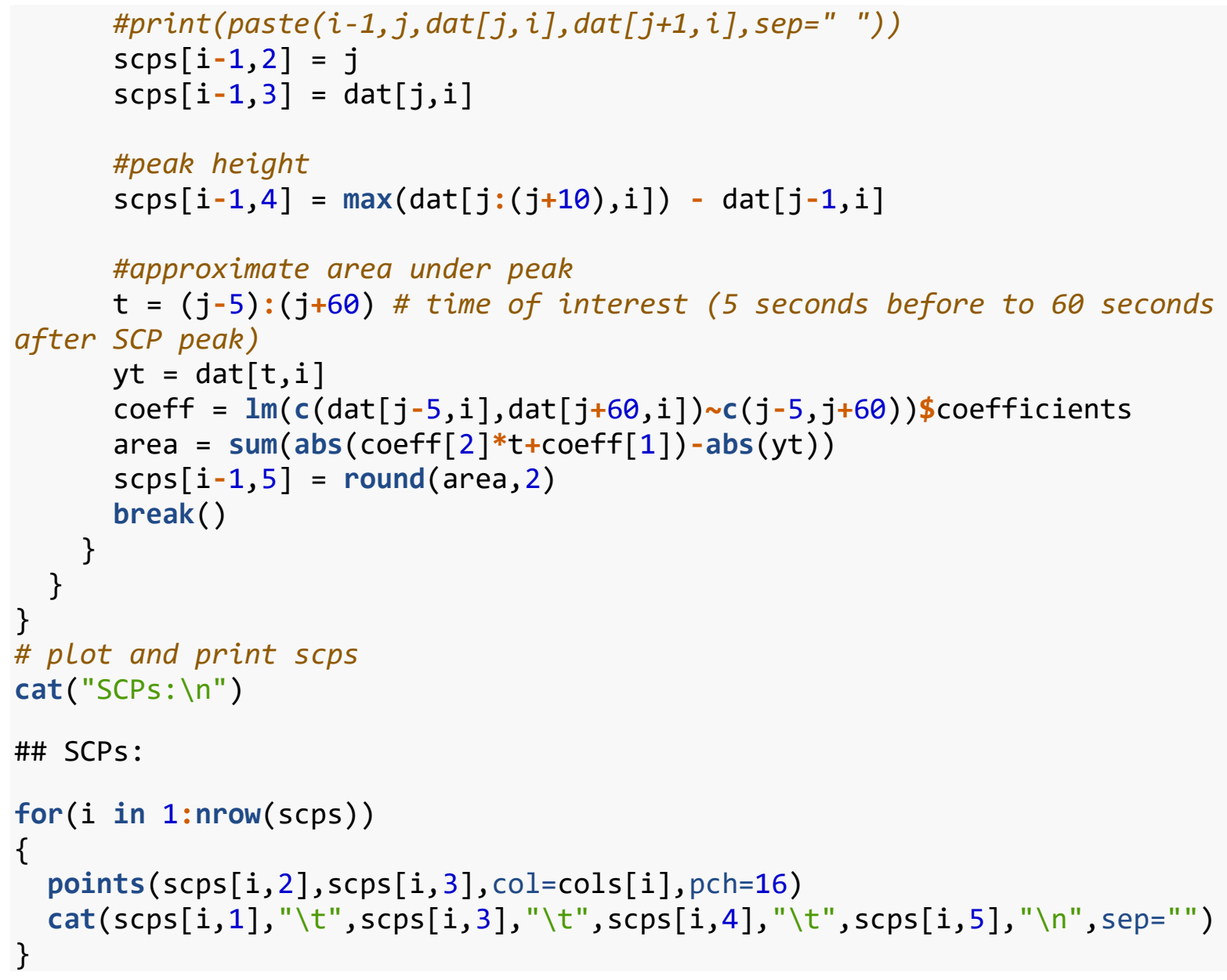




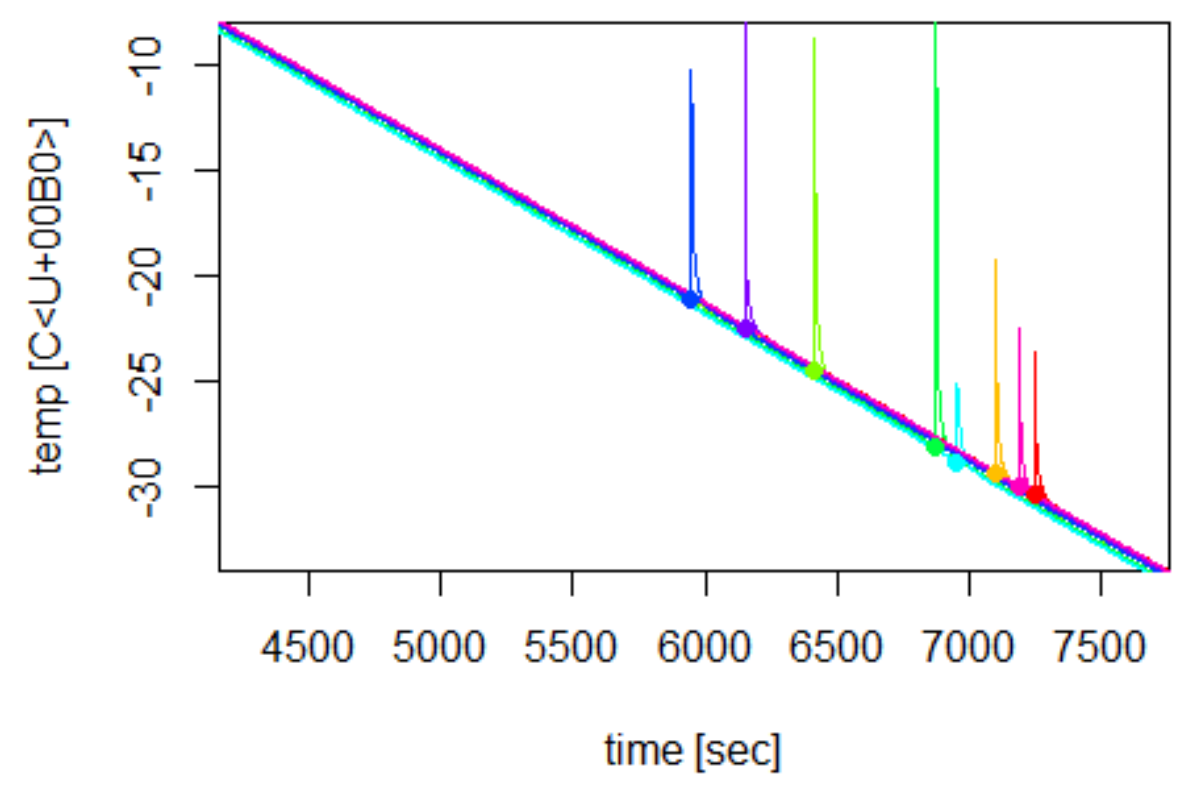

$\begin{array}{llll}\text { \#\# E18-PA-88-A } & -30.39 & 6.72 & 69.94 \\ \text { \#\# E18-PA-88-B } & -29.44 & 10.17 & 75.56 \\ \text { \#\# E18-PA-88-C } & -24.55 & 15.81 & 93.59 \\ \text { \#\# E18-PA-88-D } & -28.13 & 20.08 & 114.97 \\ \text { \#\# E18-PA-88-E } & -28.83 & 3.72 & 57.46 \\ \text { \#\# E18-PA-88-F } & -21.12 & 10.88 & 86.12 \\ \text { \#\# E18-PA-88-G } & -22.47 & 15.52 & 82.95 \\ \text { \#\# E18-PA-88-H } & -29.97 & 7.45 & 71.83\end{array}$

After obtaining the SCPs, we copied them into our larger dataset writeClipboard(scps[,3]) 


\section{Supplemental File 3}

\section{Script for Environmental Principle Components Analysis}

By: Monica M. Sheffer

\section{Calculate principle components to summarize environmental variation across sampling sites}

First, load libraries, read and filter the data

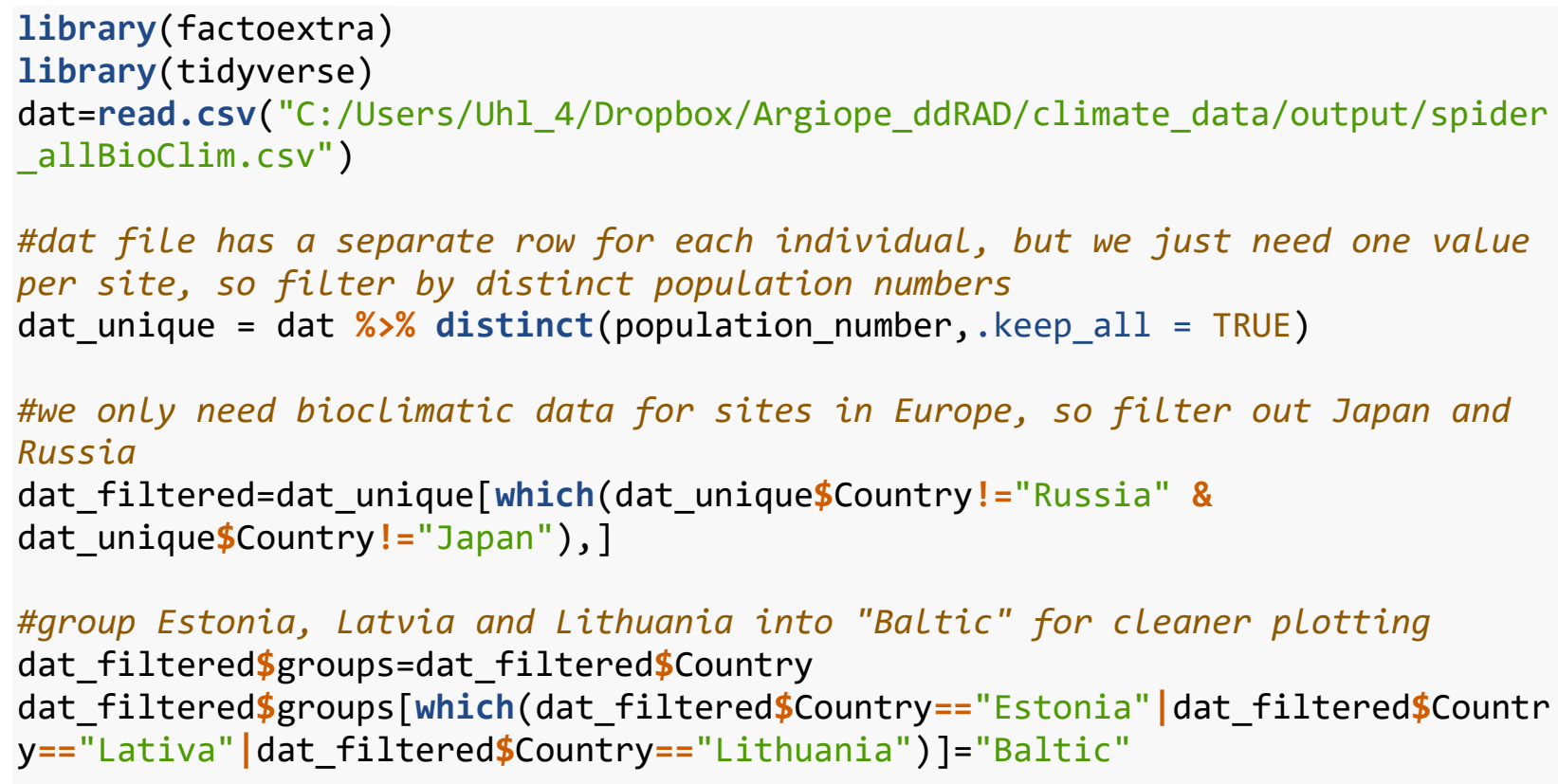


bioRxiv preprint doi: https://doi.org/10.1101/2021.11.04.467261; this version posted November 4, 2021. The copyright holder for this preprint (which was not certified by peer review) is the author/funder, who has granted bioRxiv a license to display the preprint in perpetuity. It is made available under aCC-BY-ND 4.0 International license.

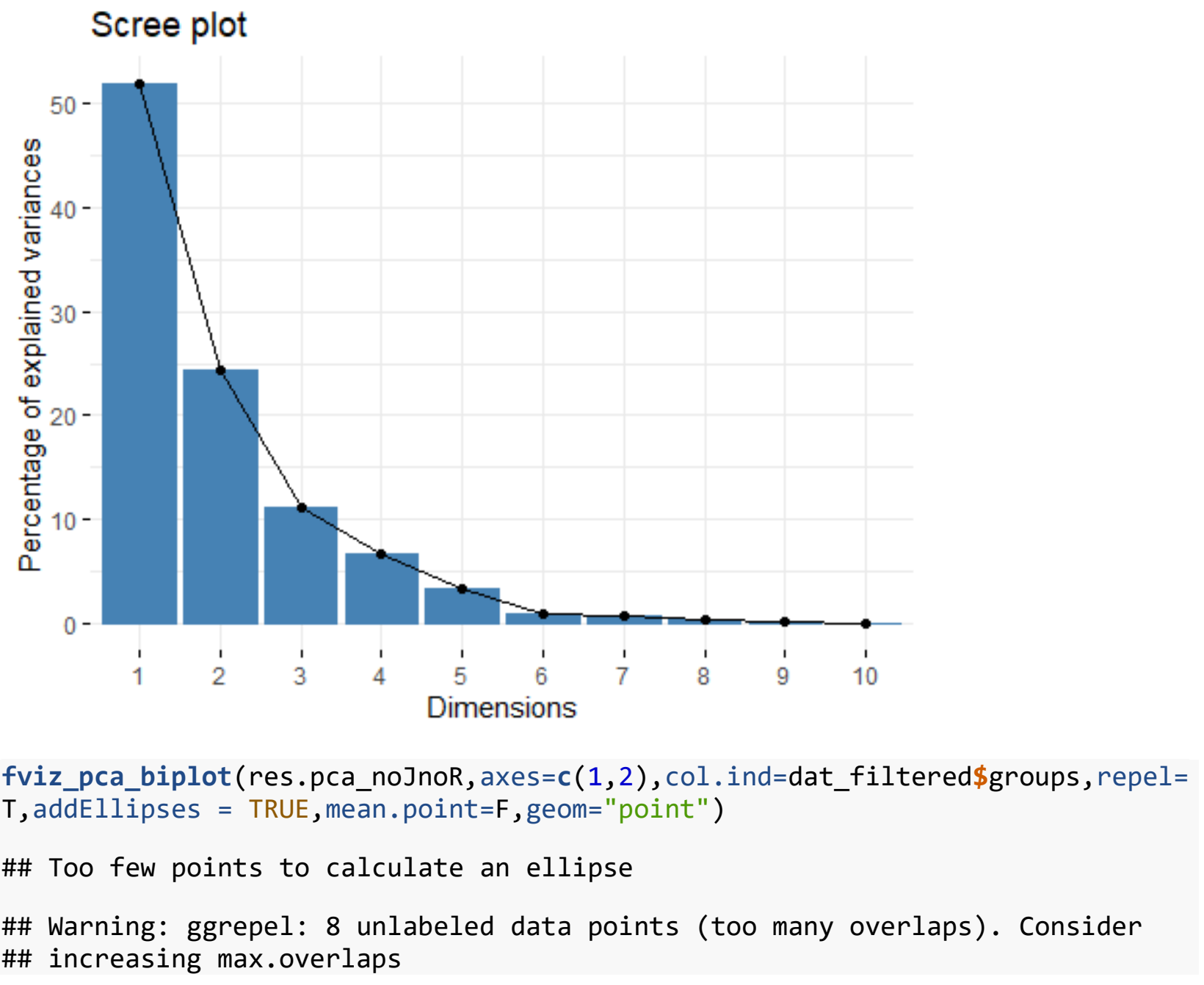




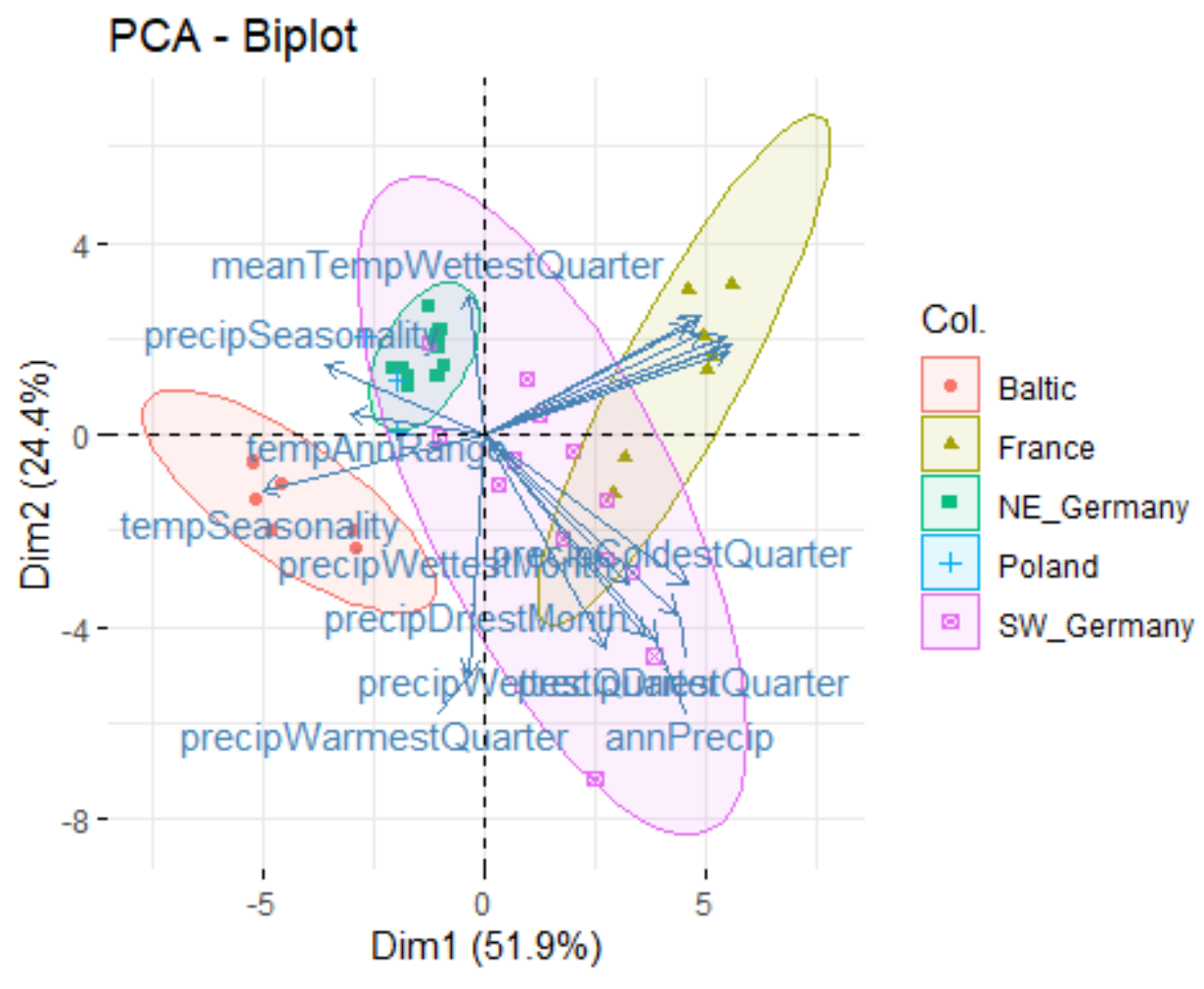

fviz_pca_biplot (res.pca_noJnoR, axes=c $(2,3), \operatorname{col}$.ind=dat_filtered\$groups, repel= $\mathrm{T}$, addEllipses $=$ TRUE, mean. point $=\mathrm{F}$, geom $=$ "point")

\#\# Too few points to calculate an ellipse 


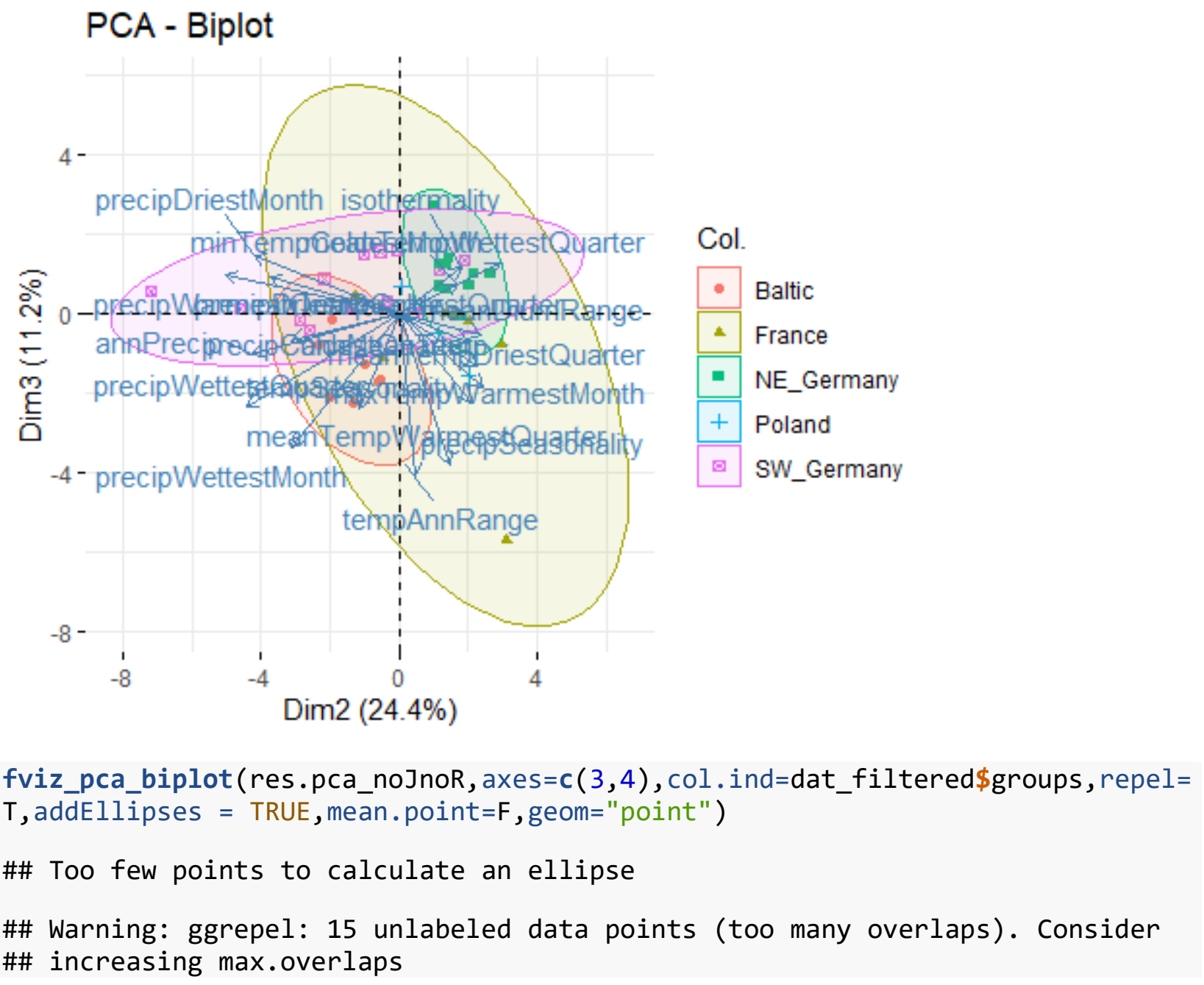




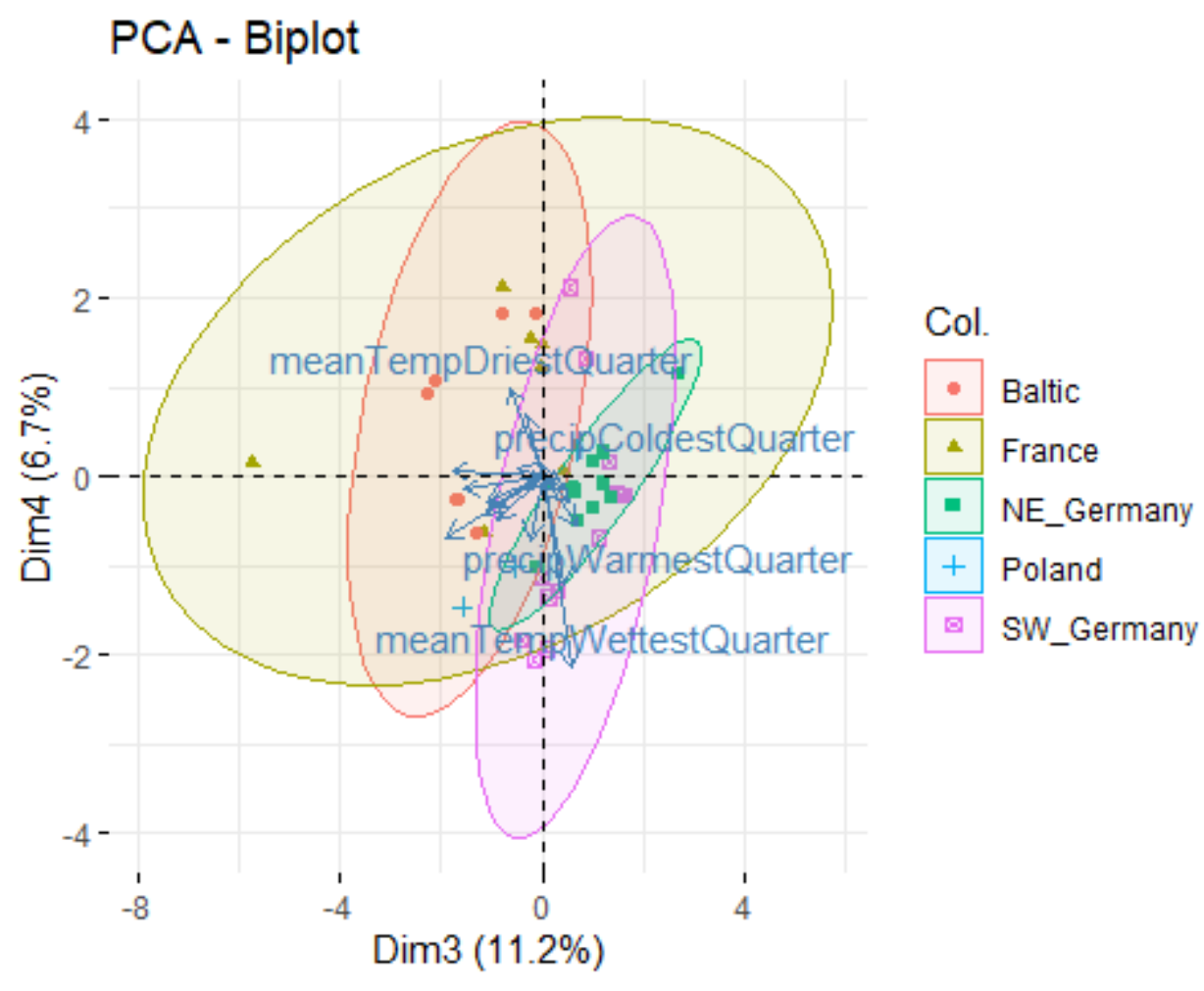

fviz_pca_biplot (res.pca_noJnoR, axes=c $(4,5)$, col.ind=dat_filtered $\$$ groups, repel= $\mathrm{T}$, addEllipses $=$ TRUE, mean. point $=\mathrm{F}$, geom="point")

\#\# Too few points to calculate an ellipse

\#\# Warning: ggrepel: 17 unlabeled data points (too many overlaps). Consider \#\# increasing max.overlaps 


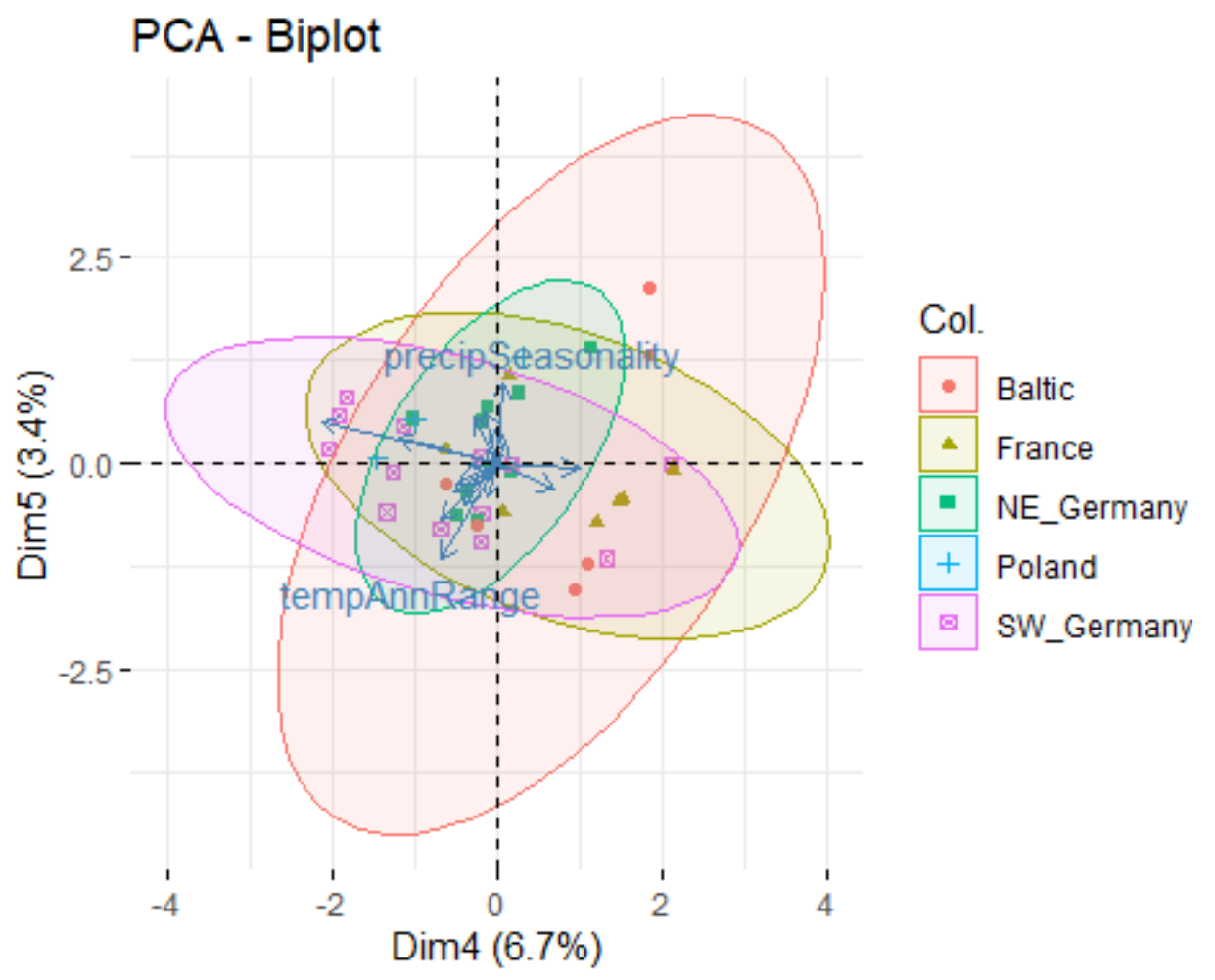

Print specific loadings of the PCs

sort (res.pca_no]noR[["rotation"] ][, "PC1"])

\#\# tempSeasonality

\#\# $\quad-0.27102370$

\#\# meanTempWettestQuarter

\#\# $\quad-0.01920380$

\#\# precipwettestMonth

\#\# $\quad 0.17512415$

\#\# precipDriestQuarter

\#\# $\quad 0.23528249$

\#\# meanDiurnRange

\#\# $\quad 0.25802749$

\#\# isothermality

\#\# 0.28742502

\#\# meanTempColdestQuarter

\#\# $\quad 0.29975360$

precipseasonality

$-0.19418552$

precipWarmestQuarter $-0.01890199$

precipDriestMonth

0.19302953

precipColdestQuarter

0.24663256

maxTempWarmestMonth

0.26150117

annMeanTemp

0.29361680
tempAnnRange

$-0.16450314$

precipwettestQuarter

0.14805015

annPrecip

0.20868676

meanTempDriestQuarter

0.24753941

meanTempWarmestQuarter

0.26204398

minTempColdestMonth

0.29645808

sort (res.pca_noJnoR[["rotation"]][, "PC2"])

\#\# precipWarmestQuarter

\#\# $\quad-0.39690373$

\#\#

\#\#

\#\#

\#\#
precipDriestMonth

$-0.33073533$

precipWettestMonth

$-0.24307533$
precipwettestQuarter

$-0.34801214$

precipDriestQuarter

$-0.29354691$

tempSeasonality

$-0.09048024$
annPrecip

$-0.33588406$

precipColdestQuarter

$-0.24590297$

tempAnnRange

0.03506497 


\begin{tabular}{|c|c|c|c|}
\hline \#\# & precipSeasonality & minTempColdestMonth & isothermality \\
\hline \#\# & 0.11606285 & 0.13427019 & 0.14388219 \\
\hline \#\# & meanTempColdestQuarter & annMeanTemp & meanTempWarmestQuarter \\
\hline \#\# & 0.14598218 & 0.15910666 & 0.16629178 \\
\hline \#\# & meanTempDriestQuarter & meanDiurnRange & maxTempWarmestMonth \\
\hline \#\# & 0.17917805 & 0.18794679 & 0.19266831 \\
\hline \multicolumn{4}{|c|}{ \#\# meanTempWettestQuarter } \\
\hline \#\# & 0.23017698 & & \\
\hline \multicolumn{4}{|c|}{ sort(res.pca_no]noR[["rotation"]][, "PC3"]) } \\
\hline \#\# & tempAnnRange & precipSeasonality & precipWettestMonth \\
\hline \#\# & -0.475428324 & -0.444724070 & -0.390397428 \\
\hline \#\# & tempSeasonality & precipWettestQuarter & meanTempWarmestQuarter \\
\hline \#\# & -0.281000718 & -0.273090805 & -0.261345177 \\
\hline \#\# & maxTempWarmestMonth & meanTempDriestQuarter & annPrecip \\
\hline \#\# & -0.217642295 & -0.155451097 & -0.117161640 \\
\hline \#\# & annMeanTemp & precipColdestQuarter & meanDiurnRange \\
\hline \#\# & -0.086738727 & -0.078688021 & -0.060974214 \\
\hline \#\# & meanTempColdestQuarter & minTempColdestMonth & precipDriestQuarter \\
\hline \#\# & 0.002638099 & 0.083023900 & 0.104256699 \\
\hline \#\# & precipWarmestQuarter & isothermality & meanTempWettestQuarter \\
\hline \#\# & 0.113134186 & 0.136119231 & 0.145361505 \\
\hline \#\# & precipDriestMonth & & \\
\hline \#\# & 0.170342286 & & \\
\hline \multicolumn{4}{|c|}{$\operatorname{sort}($ res.pca_no]noR[["rotation"]][, "PC4"]) } \\
\hline \#\# & eanTempWettestQuarter & precipWarmestQuarter & meanDiurnRange \\
\hline \#\# & -0.69162758 & -0.39009622 & -0.22923774 \\
\hline \#\# & tempAnnRange & precipDriestMonth & maxTempWarmestMonth \\
\hline \#\# & -0.22438006 & -0.17408335 & -0.16097378 \\
\hline \#\# & tempSeasonality & meanTempWarmestQuarter & isothermality \\
\hline \#\# & -0.13759165 & -0.11523039 & -0.09492883 \\
\hline \#\# & precipWettestQuarter & precipWettestMonth & precipDriestQuarter \\
\hline \#\# & -0.09394129 & -0.04957857 & -0.04437050 \\
\hline \#\# & annMeanTemp & minTempColdestMonth & precipSeasonality \\
\hline \#\# & -0.04433973 & -0.00712530 & 0.02247883 \\
\hline \#\# & meanTempColdestQuarter & annPrecip & precipcoldestQuarter \\
\hline \#\# & 0.03166551 & 0.03238765 & 0.22098703 \\
\hline \#\# & meanTempDriestQuarter & & \\
\hline \#\# & 0.32012586 & & \\
\hline \multicolumn{4}{|c|}{ sort(res.pca_noJnoR[["rotation"]][, "PC5"]) } \\
\hline \#\# & tempAnnRange & meanDiurnRange & tempSeasonality \\
\hline \#\# & -0.52716728 & -0.31030178 & -0.22280938 \\
\hline \#\# & precipDriestQuarter & precipDriestMonth & precipColdestQuarter \\
\hline \#\# & -0.18770326 & -0.15034559 & -0.14219453 \\
\hline \#\# & maxTempWarmestMonth & isothermality & annPrecip \\
\hline \#\# & -0.10805523 & -0.10269024 & -0.02936224 \\
\hline
\end{tabular}


bioRxiv preprint doi: https://doi.org/10.1101/2021.11.04.467261; this version posted November 4, 2021. The copyright holder for this preprint (which was not certified by peer review) is the author/funder, who has granted bioRxiv a license to display the preprint in perpetuity. It is made available under aCC-BY-ND 4.0 International license.

$\begin{array}{rrr}\# \# & \text { meanTempDriestQuarter } & \text { meanTempWarmestQuarter } \\ \# \# & -0.02144636 & -0.01313582 \\ \# \# & \text { meanTempColdestQuarter } & \text { precipWarmestQuarter } \\ \# \# & 0.12209270 & 0.13787975 \\ \# \# \text { meanTempWettestQuarter } & \text { precipWettestQuarter } \\ \# \# & 0.23263255 & 0.25508775 \\ \# \# & \text { precipSeasonality } & \\ \# \# & 0.44035083 & \end{array}$

annMeanTemp

0.07925481

minTempColdestMonth

0.19799805

precipWettestMonth

0.28617036 